\title{
UNSTEADY FORCE ESTIMATION \\ USING A LAGRANGIAN DRIFT-VOLUME APPROACH
}

by

\section{Cameron James McPhaden}

\author{
A thesis submitted to the \\ Department of Mechanical and Materials Engineering \\ in conformity with the requirements for \\ the degree of Master of Applied Science
}

Queen's University

Kingston, Ontario, Canada

August 2017

Copyright (C) Cameron James McPhaden, 2017 


\begin{abstract}
A novel Lagrangian force estimation technique for unsteady fluid flows has been developed, using the concept of a Darwinian drift volume to measure the unsteady force on an accelerating body. A methodology using multiple drift volumes is described and evaluated on an experimental test case containing highly-separated, vortical flow. The inherent advantage of the force estimation technique presented is that, unlike many modern Eulerian techniques, gradient operations and near-body measurements are not required to be calculated. These noise amplifying processes are avoided since the drift volume is calculated only from particle displacements in the flow field, reducing the importance of having high quality acceleration and spatial gradient data near walls and in regions of high shear. The resultant unsteady force estimates from the proposed technique are shown to align with the measured drag force during high accelerations, a region in which comparable methods suffer. The critical aspects of understanding unsteady flows, relating to peak and time-resolved forces, often lie within the acceleration phase of the motions, which are well-captured by the drift-volume approach. Therefore, this Lagrangian force estimation technique opens the door to fluid-dynamic analyses in areas that, until now, were inaccessible by conventional means.
\end{abstract}




\section{Acknowledgments}

Above all else, I would like to thank my supervisor, Dr. David Rival, for his knowledge and teaching, for affording me the opportunities to learn and collaborate with the world's best, for his patience and understanding, and for making me learn the hard way that nothing is ever perfect.

I would like to thank all my research group colleagues, specifically Drs. John Fernando, Giuseppe Rosi and Amirreza Rouhi, for their guidance and constructive conversations over the past years. Special thanks goes out to Dr. Jaime Wong for his assistance and dedication of time toward my research.

I must also acknowledge the financial support from the Ontario Graduate Scholarship and the Natural Sciences and Engineering Research Council of Canada, which made this work possible.

I would like extend a sincere thank you to the entire First Capital Cycling community for taking me in as one of their own. In particular, I owe a large debt of gratitude to Ka-Yu, Matt, Andy, Jim and Sue for being quality role models, pushing my limits, and challenging me to always become a better version of myself.

To my family: Thank you for your unwavering support in all aspects of my life over the course of the last few years. Without you all, I would not have made it to where I am today. 


\section{Contents}

Abstract $\quad$ i

$\begin{array}{ll}\text { Acknowledgments } & \text { ii }\end{array}$

Contents

List of Tables $\quad$ V

List of Figures $\quad$ vi

List of Abbreviations, Acronyms and Symbols xiii

Chapter 1: Introduction 1

1.1 Motivation . . . . . . . . . . . . . . . . . 1

1.2 Current Force Measurement Limitations . . . . . . . . . . . . . . . 2

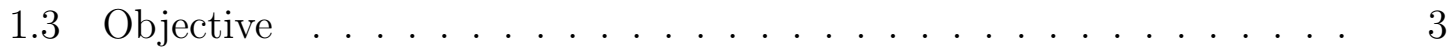

1.4 Thesis Outline. . . . . . . . . . . . . . . . 3

Chapter 2: $\quad$ Background 5

2.1 Contemporary Fluid-Force Estimation Techniques . . . . . . . . . . 5

2.2 Formulations for Total Fluid Force . . . . . . . . . . . . . . . . . 7

2.3 Added-Mass Force . . . . . . . . . . . . . . . . . . . . . 10

2.3.1 Coefficient of Added Mass . . . . . . . . . . . . . . . . . . 14

2.3.2 Time-Varying Added Mass . . . . . . . . . . . . . . . . . . 16

2.4 Force Formulations from Vorticity . . . . . . . . . . . . . . . . 16

2.5 Rotational and Irrotational Force Decomposition . . . . . . . . . . . . 19

2.6 Lagrangian Force Estimation Techniques . . . . . . . . . . . . . . . . 20

2.7 Drift Volume and Darwin's Proposition . . . . . . . . . . . . . . 21

Chapter 3: $\quad$ Proposed Force Estimation Technique 28

3.1 Description of Test Case . . . . . . . . . . . . . . . . . . 28

3.2 Single-Plane Drift-Volume Calculation . . . . . . . . . . . . . . . 31 
3.3 Multi-Plane Drift-Volume Calculation . . . . . . . . . . . . . 33

$\begin{array}{lll}\text { Chapter 4: } & \text { Experimental Methods } & 37\end{array}$

4.1 Force measurements . . . . . . . . . . . . . . . . . . 37

4.2 Particle image velocimetry measurements . . . . . . . . . . . . 39

Chapter 5: Results and Discussion $\quad 45$

5.1 Multi-Plane Drift Volume . . . . . . . . . . . . . . . . . . . 45

5.2 Force Analysis . . . . . . . . . . . . . . . . . . . . . . . . . . . 48

5.2.1 Transition Toward Steady State . . . . . . . . . . . . . 51

5.2.2 Comparison to Modern Eulerian Methods . . . . . . . . . . 52

5.2.3 Direct Comparison to the Wake-Vortex Method . . . . . . . . 56

5.2 .4 Force Estimation Artifacts . . . . . . . . . . . . . . . 58

$\begin{array}{lll}\text { Chapter 6: } & \text { Conclusions } & 61\end{array}$

6.1 Conclusions . . . . . . . . . . . . . . . . . . . . . 61

6.2 Future Experimental Considerations . . . . . . . . . . . . . . . . 64

6.3 Future Work . . . . . . . . . . . . . . . . . . . . 65

$\begin{array}{ll}\text { Bibliography } & 67\end{array}$

$\begin{array}{ll}\text { Appendix A: Uncertainty Analysis } & 76\end{array}$

A.1 PIV Uncertainty . . . . . . . . . . . . . . . 76

A.2 Measured force uncertainty . . . . . . . . . . . 77

$\begin{array}{ll}\text { Appendix B: MATLAB Code } & \mathbf{7 8}\end{array}$

B.1 Algorithm Description . . . . . . . . . . . . . . . . . 78

B.2 Calculation of Drift Volume . . . . . . . . . . . . . . . 79

B.3 Force Calculation . . . . . . . . . . . . . . . . . . . 91 


\section{List of Tables}

2.1 Overview of error sources for the examined Eulerian force-extraction techniques. Each multiplication, temporal derivative and spatial derivative propagates errors throughout the calculation. Table adapted from Rival and Oudheusden (2017). . . . . . . . . . . . . .

5.1 The accuracy of the Lagrangian drift-volume force estimation technique is compared to the aforementioned Eulerian force estimation techniques. Each multiplication, temporal derivative and spatial derivative propagates errors throughout the calculation. The drift-volume technique has the lowest value in three of the four columns of error

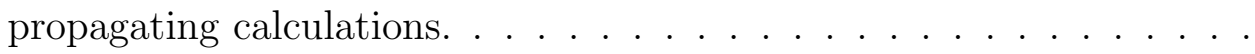




\section{List of Figures}

2.1 The domain of integration (blue) for the evaluation of fluid-dynamic forces on a body (orange) is illustrated. Volumetric quantities are integrated over the control volume $(\mathrm{CV})$ and surface quantities are integrated over the control surface (CS). The direction of normal vectors $\hat{\mathbf{n}}$ are shown for both the outer CS and the body control surface $\left(\mathrm{CS}_{b}\right)$.

2.2 A) A wheel and mud illustration represents Darwin's added-mass analogy. A wheel picks up mud, representing the added mass, from the road and sets it back down after one rotation, changing only its displacement. B) A quasi-continuous system of mud on a wheel represents a closer analogy to the added-mass effects of a body moving through a fluid. Although the mud imparts no net force onto the wheel during constant velocity operation, the mud (added mass) influences the required force necessary to accelerate the wheel (submersed body). .

2.3 Added-mass coefficients are derived from potential flow theory for ellipsoids (solid line) and elliptical cylinders (dashed line) for varying length to diameter ratios. The length to diameter ratio is the ratio of the axis parallel to the direction of motion to the axis perpendicular to the direction of motion. Figure from Daniel (1984). . . . . . . . 
2.4 Illustration of a drift volume, as defined by Darwin (1953). A body, initially upstream of a reference plane of tracked fluid, passes through the plane of fluid, bending it around the body. Fluid initially upstream of the reference plane undergoes a net downstream displacement, known

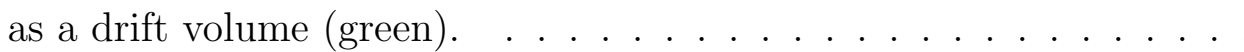

2.5 Potential flow streamlines around a sphere in steady flow. Viewed from a reference frame attached to a sphere at constant velocity, the movement of fluid around the sphere is described by stationary streamlines. The narrowing gaps between streamlines around $y / R= \pm 1$ indicate higher streamwise velocity, producing fluid that has a velocity magnitude larger than that of the translating sphere, but in the opposite direction. This phenomenon is better visualized in Figure 2.6. . . . .

2.6 Contours of $x$-direction velocity are shown for the potential flow solution of a sphere translating in steady flow. The flow moves from left to right around a stationary sphere (white). In the blue regions, flow is slowed down relative to the sphere, bringing the fluid closer to rest. In red regions, fluid is accelerated around the sides of the sphere to a velocity greater than the free stream velocity. This downstream acceleration of fluid suggests that flow reversal occurs if viewed from a stationary (ambient) frame of reference. Particle pathlines in Figure 2.7 show these flow reversal events clearly. . . . . . . . . . . 
2.7 Particle pathlines, coloured by time, are shown for the potential flow solution of a constant velocity sphere traveling in the $+x$-direction. Each pathline originates from the $x / R=0$ plane (within a tolerance of $|x / R| \leq 0.05)$, and is convected downstream. Flow reversal events, called elasticas, form loops in the particle pathlines due to the regions of increased relative velocity around the sides of the sphere. The drift volume associated with a potential flow sphere is viewable as the region between particles at $t=0$ (blue) and $t \gg 0$ (red) . . . . . .

3.1 Force versus acceleration modulus showing varying peak force. Each curve represents an accelerating case where a circular flat plate starts from rest and accelerates to the final towing velocity $U_{f}$, but over varying time intervals. The relationship between acceleration modulus $a^{*}$ and acceleration time $t_{a}^{*}$ is inversely proportional: $t_{a}^{*}=1 / a^{*}$. Figure adapted from Fernando (2017). . . . . . . . . . . . .

3.2 (top) The velocity profile of the accelerating plate is prescribed over time. Initially at rest, the plate undergoes constant acceleration at $a^{*}=$ 4 until the final towing velocity $U_{f}$ is reached at $t^{*}=0.25$, where it then maintains constant velocity. (bottom) The drag coefficient is measured using a physical force sensor. The plate's acceleration produces a force peak at $t^{*}=0.25$ due to added-mass effects. . . . . . . . 
3.3 Pathlines are shown for synthetically seeded particles that originate upstream $(+x)$ of a reference plane placed at $x / D=0$. Tracks are shown only for particles that cross the reference plane, traveling from upstream of the reference plane $(+x)$ to downstream of the reference plane $(-x)$ when $t \gg 0$. For enhanced clarity, only $10 \%$ of tracks are

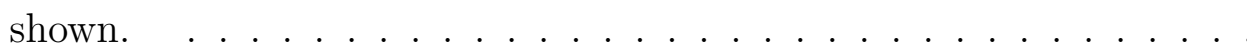

3.4 Drift-volume development is shown for four arbitrary chosen reference planes, equally spaced from $x / D=-1.75$ to $x / D=0.5$ at $t^{*}=2$. The amount of green (positive drift) fluid is noticeably different depending on reference plane location. In each case, the plate's position is $x / D \approx$ $-1.5 . \ldots \ldots \ldots \ldots \ldots \ldots \ldots \ldots \ldots \ldots \ldots \ldots \ldots \ldots \ldots$

3.5 Illustration of multi-plane drift-volume calculation. The total drift volume $\Omega_{d}$ is the sum of the individual drift-volume contributions $\Omega_{d_{n}}$ from many single reference planes. . . . . . . . . . . . .

3.6 Double accounting of drift volume can occur when the drift volumes developed from two successive reference planes overlap. A) and B) show individual drift volume contributions $\Omega_{d_{n}}$ to the total drift volume $\Omega_{d}$. C) shows the overlapping region within which double accounting can occur. D) shows the rectified total drift volume $\Omega_{d}$, limiting the calculation of the drift volume in A) to within the region bounded by its neighbouring reference plane. $\ldots \ldots \ldots \ldots \ldots$

4.1 The towing-tank facility in the OTTER Lab at Queen's University measures $15 \mathrm{~m}$ in length, has a $1 \mathrm{~m} \times 1 \mathrm{~m}$ cross-section, and contains a slotted roof with a high-speed traverse system. 
4.2 Towing-tank facility depicting the circular flat plate within the tank, and the location of the force transducer (shown in the inset). . . .

4.3 The experimental set-up for PIV measurements, including the location of the high-speed laser and camera, and the approximate size and orientation of the field of view $(\mathrm{FoV}) . \ldots \ldots$

4.4 Multiple fields of view for PIV measurements were arranged in the streamwise direction. In reality, the laser and the camera remained stationary and the plate's starting position was altered to change the effective FoV captured. Red dashed lines represent locations where there exists a $10 \%$ overlap between FoVs, however neighbouring FoVs have been separated for illustrative clarity. . . . . . . . . . . .

4.5 Cylindrical integration around the plate's axis of symmetry is calculated for individual fluid particles. Particle pathlines (blue lines) are shown for particles (blue spheres) originating from a single reference plane. Integration pathlines (red dashed lines) are shown for a fluid particle at three distinct timesteps. The laser sheet (green) is shown

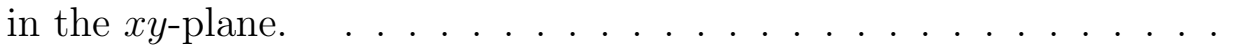

5.1 Positive drift (green) and negative drift (red) are shown for a varying number of reference planes $N$ at various times $t^{*}$. Shown here are illustrations for $N=20$ (left), $N=40$ (middle), and $N=80$ (right). Multi-plane drift is shown for $t^{*}=0.13(A), t^{*}=0.25(B)$, and $t^{*}=$ $0.38(C)$. The plate, initially at $x / D=0$ with its axis of symmetry located on the $r / D=0$ plane, accelerates from rest at in the $-x$ direction and over the time interval $0<t^{*}<0.25$. 
5.2 Forces are calculated from the multi-plane drift-volume approach for a varying number of reference planes $N$. The measured force from a physical force transducer (black dashed line) is shown for comparison. The classical added-mass force from potential flow (blue) is directly proportional to the acceleration of the body; however, it does not match up with the measured force peak. The acceleration occurs over the time interval $0<t^{*}<0.25 \ldots \ldots \ldots \ldots \ldots \ldots \ldots$

5.3 Measured force (black) is compared to the multi-plane drift-volume technique $(r e d)$. During the acceleration period, $0 \leq t^{*} \leq 0.25$, the drift-volume method follows the measured force curve with good agreement. After $t^{*}=0.25$, the discrepancy between the two curves increases beyond the limits of experimental error. The measured force curve is not well tracked in its relaxation toward state-state conditions. 51

5.4 Measured force (black line) is compared to two different methods of estimated force: the multi-plane drift-volume technique (red) and a wake-vortex formulation from $\mathrm{Wu}$ et al. (2006) (blue). The dashed colored lines represent the region for each technique where the force estimate is no longer accurate, based on known limitations of the measurement techniques. The state of the wake is shown for two times, $t^{*}=0.25$ and $t^{*}=0.5$, where the colours, blue and red, in the inset images represent positive and negative $z$-direction vorticity $\omega_{z}$, respectively. $\ldots \ldots \ldots \ldots \ldots \ldots \ldots \ldots \ldots$ 
5.5 Error is induced in the drift-volume calculation when fluid particles displaced in the streamwise direction are outside of the field of view. In this illustration, the negative drift volume contribution is underestimated. . . . . . . . . . . . . . . . . .

5.6 Two values of $N$ are compared to show the artificial force increase associated with lower $N$ for the case where displaced fluid in the start up vortex is not captured until later in time. Closer reference plane spacing, higher $N(\mathrm{~A}, \mathrm{~B}, \mathrm{C})$, is compared to larger reference plane spacing, lower $N(\mathrm{D}, \mathrm{E}, \mathrm{F})$. When the plate accelerates from rest, as shown in the progression from top row to bottom row, smaller values of $N$ do not capture the downstream fluid displacement contained within the starting vortex (E versus B). Later in time, the vortex passes through the next downstream reference plane, and a rapid increase in the measured drift volume occurs (F). This increase results in an artificial peak in the $\partial m_{a} / \partial t$ term of Equation 2.8, whereas this peak does not occur for large $N$ since this drift was previously captured (C). . . . . . . 60 


\section{List of Abbreviations, Acronyms and Symbols}

\section{Acronyms}

$\begin{array}{ll}\text { CS } & \text { Control surface, } \\ \text { CV } & \text { Control volume, } \\ \text { DMT } & \text { Derivative-moment transformation, } \\ \text { FoV } & \text { Field of view, } \\ \text { Nd:YLF } & \text { Neodymium-doped yttrium lithium fluoride (lasing medium), } \\ \text { PIV } & \text { Particle image velocimetry, } \\ \text { Tomo-PIV } & \text { Tomographic particle image velocimetry, } \\ \text { LPT } & \text { Lagrangian Particle Tracking } \\ \text { STB } & \text { Shake-The-Box, }\end{array}$

\section{Symbols}

\section{Latin}

$a$

$\cdot a^{*}$

A

$c_{i i}$
Acceleration,

Dimensionless acceleration (linear),

Frontal area of circular plate,

Added mass coefficient, 


$\begin{array}{ll}C_{D} & \text { Coefficient of drag, } \\ d_{c} & \text { Characteristic length scale, } \\ d_{d} & \text { Seeding particle diameter, } \\ D & \text { Diameter, } \\ F & \text { Force, } \\ H & \text { hydrodynamic impulse, } \\ I & \text { Unit tensor, } \\ m_{a} & \text { Added mass, } \\ N & \text { Number of reference planes, } \\ \hat{n} & \text { Unit normal vector, } \\ p x & \text { Pixel, } \\ p p p & \text { Particles per pixel, } \\ R & \text { Radius of sphere, } \\ \text { Re } & \text { Reynolds number, } \\ \text { Stk } & \text { Stokes number, } \\ t & \text { Time, } \\ T & \text { Viscous stress tensor, } \\ \cdot t^{*} & \text { Dimensionless time, } \\ u, v, w & \text { Velocity components, } \\ \cdot U_{f} & \text { Final towing velocity, } \\ V_{\infty} & \text { Free-stream velocity, } \\ x, y, z & \text { Cisplaced fluid volume, } \\ V_{b}, \theta, z & \text { Cylindrical coordinates } \\ & \end{array}$

xiv 


\section{Greek}

$\begin{array}{ll}\nu & \text { Kinematic viscosity, } \\ \mu & \text { Dynamic viscosity, } \\ \rho & \text { Density, } \\ \rho_{d} & \text { Seeding particle density, } \\ \Gamma & \text { Circulation, } \\ \omega_{x}, \omega_{y}, \omega_{z} & \text { Vorticity coordinates in } x, y \text { and } z, \\ \Omega_{d} & \text { Drift volume, } \\ \cdot \Omega_{d_{n}} & \text { Single-plane drift-volume contribution, } \\ \Omega_{V} & \text { Wake-vortex volume, } \\ \tau & \text { Relaxation time }\end{array}$




\section{Chapter 1}

\section{Introduction}

\subsection{Motivation}

Fluid force estimates are of great interest in many areas of fluid dynamics. They serve to provide information in the realms of aerodynamic loading (Motta et al. (2015) and Wang et al. (2016)), fluid-structure interactions (Garca et al. (2017) and Ahsan and Aureli (2017)), the dynamics of natural swimmers and flyers (Bos et al. (2008) and Eloy (2013)), as well as to provide a design basis for engineering development; for example, modern interests include micro aerial vehicles (Ansari et al. (2006) and Benedict et al. (2016)). The ability to measure instantaneous dynamic loading gives physical insight into the fluid mechanics responsible for force generation. Unfortunately, to measure fluid dynamic loads directly, a physical force transducer placed within the flow is required, which may alter the dynamics of the measurements. In addition to disrupting the fluid flow, it is often unrealistic to attach a physical force sensor to a body that is either too small, too lightweight, or has delicate interactions

with its fluid environment. For instance, the study of biomimetic applications in fluid dynamics explores the natural flying and swimming associated with birds, fish, 
insects and other small animals, on which using a physical force transducer can be impractical due to physical limitations. The alternative to direct force measurement is to use non-intrusive, optical methods. Since the force on a body cannot be measured directly with optical techniques, the governing fluid dynamics equations are instead used to extract forces from optical flow field measurements.

\subsection{Current Force Measurement Limitations}

Non-intrusive fluid flow measurements have developed considerably in recent years; steady advances in high-speed imaging have allowed for techniques such as Tomographic PIV (Tomo-PIV) (Elsinga et al. (2006)) and Shake-The-Box (Schanz et al. (2016)) to capture complete time-resolved volumetric flow data around submersed bodies of interest. Tomo-PIV is a particle tracking method that uses tomographic particle reconstructions of multiple camera angles to calculate velocities, whereas Shake-The-Box uses this same process but also makes predictions of particle locations based on previously calculated flow data along their pathlines in order to reduce computational costs and, more importantly, to eliminate fictitious particles that arise from ambiguity in classical 3D-PTV algorithms. However, these measurements are still susceptible to a finite dynamic spatial range; that is, a trade-off exists between the ability to measure small-scale fluid structures using high resolution imaging and to measure large-scale structures using a large field of view (FoV). There currently exist various Eulerian methods of non-intrusive force estimation, each with their own strengths and limitations, that are reviewed by Rival and Oudheusden (2017). The constraint of spatial resolution in regions of high shear, high vorticity and near walls poses a significant obstacle to the accurate interpretation of flow data, as described 
by Westerweel (2008) and Kähler et al. (2012). These near-wall regions can be of utmost importance since they contain the highest accelerations and velocity gradients, and are the regions that dictate the loading on a body. Because the errors associated with current measurement techniques are the highest in these regions of interest, an alternative approach using Lagrangian analysis techniques has been developed to circumvent many of the requirements that were previously necessary for accurate unsteady force estimation from non-intrusive measurements.

\subsection{Objective}

In this thesis, a new non-intrusive, force-estimation technique is described, using a Lagrangian perspective, that aims to avoid the measurement challenges associated with gradient operation errors coupled to dynamic spatial range limitations of current force estimation methods. A Lagrangian framework is used to draw information from the wake, using a drift-volume concept introduced by Darwin (1953), in an attempt to bypass the requirement of having high resolution within a large field of view. The described technique is designed to use three-dimensional time-resolved flow data obtained from Lagrangian Particle Tracking (LPT) algorithms. This type of force estimation methodology has not been fully considered in previous studies and warrants investigation.

\subsection{Thesis Outline}

In Chapter 2, contemporary fluid force measurements from optical flow field data in both Eulerian and Lagrangian reference frames are reviewed. The concept of added mass and its relationship to drift volumes are also discussed, and an argument is made 
for how the current technique fills a gap in the literature.

In Chapter 3, force calculations from drift-volume development is explored through an experimental test case of an accelerating circular plate. A new multi-plane driftvolume technique is described, which reconciles the limitations of current single-plane drift-volume force estimation methods.

In Chapter 4, the details of the accelerating circular flat plate experimental test case are discussed. The equipment used within the particle image velocimetry (PIV) experimental setup, the kinematics of the plate's motion, and synthetic Lagrangian seeding are detailed.

In Chapter 5, the drift-volume technique is evaluated against an Eulerian wakevortex method of force estimation and against the measured force from a force transducer. Calculation artifacts of the multi-plane drift-volume method are discussed in detail, describing confidence bounds for various control inputs used in the technique. The proposed technique is compared to existing force estimation methods and its strengths are outlined.

In Chapter 6, the main findings of the experimental investigation are discussed and the advantages of the proposed technique over existing force measurement techniques are reviewed to verify the relevance of the current work. Experimental recommendations are made to improve future data quality for those who attempt to build on the work of this thesis. Finally, some envisioned future work is described for the development of unsteady force estimation moving forward.

Appendix A contains uncertainty analysis for PIV-based measurements and for direct force measurements. Appendix B contains the essential MATLAB scripts required to execute the multi-plane drift-volume force estimation technique. 


\section{Chapter 2}

\section{Background}

The purpose of this chapter is to provide context and motivation for the proposed Lagrangian drift-volume, force-estimation technique. First, the current state of the field of non-intrusive force measurements is reviewed. This review identifies the aspects of existing Eulerian force-estimation methods that are incorporated into the novel Lagrangian technique presented. Simultaneously, this review demonstrates the scarcity of Lagrangian methods in the literature. Second, an argument is made for the importance of exploring methods of analysis that make use of a Lagrangian frame. This argument is predicated on the advantages of Lagrangian flow data, such as increased accuracy in velocity gradients, and the current advances in particle tracking technology.

\subsection{Contemporary Fluid-Force Estimation Techniques}

When a body, submerged in a fluid, is in motion relative to the fluid, it experiences a reaction force. The existence of such a force has been demonstrated long before the advent of fluid mechanics as a science. The force of the fluid flow on the body can be evaluated directly from the external force needed to move or hold the body 


\subsection{CONTEMPORARY FLUID-FORCE ESTIMATION TECHNIQUES}

along a trajectory. This force measurement requires a force sensor to be attached directly to the body. Alternatively, forces can be estimated from the mechanics of how fluid moves around and interacts with the submerged body. Some of the earliest force measurement methods, those of Betz (1925) and Jones (1936), relied on wakepressure reconstructions from Pitot-static point data to calculate the momentum deficit of a nominally two-dimensional flow. These pressure measurements were not only intrusive, requiring a physical sensor in the flow, but were also time-averaged so as to obtain a steady-state drag, rather than an instantaneous drag, on the body in question.

The first non-intrusive, force-estimation techniques used fluid visualizations to estimate the strength and velocity of vortical structures in the wake of a body. The relationship between the observed trajectories of vortices and the measured lift and drag on a translating cylinder was first defined qualitatively by Maull and Milliner (1978), who tracked the time-dependent loci of visualized vortices, then related them to the general variation of forces on a cylinder by use of the Blasius theorem. Using the derivation of Sarpkaya (1963) for the Blasius theorem, the force, in this case the lift $L$, on a cylinder with its longitudinal axis perpendicular to the flow can be defined as

$$
L=-\rho \sum_{k=1}^{m} \Gamma_{k}\left(-U-u_{k}+u_{k_{i}}\right)-\rho \sum_{k=1}^{m} p_{k_{i}} \frac{\partial \Gamma_{k}}{\partial t},
$$

where $U$ is the velocity of the cylinder, $\Gamma_{k}$ and $u_{k}$ are the circulation and velocity in the streamwise direction of the $k^{\text {th }}$ vortex, while $u_{k_{i}}$ and $p_{k_{i}}$ are the velocity and location of the corresponding image vortex. Additionally, Ikeda and Yamamoto (1981) used particle streak visualizations to relate the strength and trajectories of vortices in the wake to the induced lift on a cylinder. These intrinsic methods of fluid force 
estimation have the advantage over extrinsic methods in that the ability to measure sectional fluid forces is possible, relating force to the topology of the flow field, rather than having a single blind force measurement from a single transducer reading.

Following the introduction of digital PIV by Willert and Gharib (1991), there has been a sizable increase in the number of investigations into force estimation techniques that utilize optical flow field data. PIV is a method of evaluating velocity vectors from pairs of sequential camera frames that contain images of particles following a fluid flow. This velocity data, in turn, can be used to estimate the forces acting on a body that has produced a disturbance in the velocity field.

While force estimation techniques have been developed for a broad range of flows, the interest of the present work is limited to the area of incompressible, accelerating flows. In this regime, many force estimation techniques already exist, but have limitations that will be further explained.

\subsection{Formulations for Total Fluid Force}

An equation for the evaluation of forces from flow-field quantities is the momentum equation in integral form. Given an arbitrary, time-dependent control volume (CV), shown in Figure 2.1, bounded externally by a control surface (CS) and internally by the body control surface $\left(\mathrm{CS}_{b}\right)$, the fluid dynamic force $\mathbf{F}(t)$ acting on a body can be 
expressed as

$$
\begin{aligned}
\mathbf{F}(t)= & \rho \iiint_{\mathrm{CV}} \mathbf{a}_{\text {rel }} \mathrm{d} V-\rho \iiint_{\mathrm{CV}} \frac{\partial \mathbf{u}}{\partial t} \mathrm{~d} V \\
& -\rho \iint_{\mathrm{CS}} \mathbf{u}\left(\mathbf{u}_{\text {rel }} \cdot \hat{\mathbf{n}}\right) \mathrm{d} S+\iint_{\mathrm{CS}} \hat{\mathbf{n}} \cdot(-p \mathbf{I}+\mathbf{T}) \mathrm{d} S \\
& -\rho \iint_{\mathrm{CS}_{b}} \hat{\mathbf{n}} \cdot\left(\mathbf{u}-\mathbf{u}_{S}\right) \mathbf{u} \mathrm{d} S,
\end{aligned}
$$

where $\hat{\mathbf{n}}$ is a unit vector, $\mathbf{u}$ is the flow velocity, $\mathbf{u}_{S}$ is the body wall velocity, $p$ the pressure, $\mathbf{I}$ the unit tensor, and $\mathbf{T}$ the viscous stress tensor for an incompressible flow:

$$
\mathbf{T}=\mu\left(\nabla \mathbf{u}+\nabla \mathbf{u}^{T}\right),
$$

where $\mu$ is the dynamic viscosity. For solid objects (i.e. not permeable by the fluid), the surface integral over the body is null for the no-through-flow condition. Additionally, the acceleration and mass flux terms are a relative quantity, being able to account for relative angular and linear acceleration effects in a moving reference frame. If an inertial control volume is used and the body is non-permeable, Equation 2.2 simplifies to:

$$
\mathbf{F}(t)=-\rho \iiint_{\mathrm{CV}} \frac{\partial \mathbf{u}}{\partial t} \mathrm{~d} V-\rho \iint_{\mathrm{CS}} \mathbf{u}(\mathbf{u} \cdot \mathbf{n}) \mathrm{d} S+\iint_{\mathrm{CS}} \hat{\mathbf{n}} \cdot(-p \mathbf{I}+\mathbf{T}) \mathrm{d} S,
$$

henceforth referred to as the classical formulation. The control surface integrals in Equation 2.4 (convective, pressure, and viscous terms) can be neglected in cases where the control volume extends sufficiently far away from the body so that ambient conditions neutralize the control surface integration. Additionally, for a fixed control 


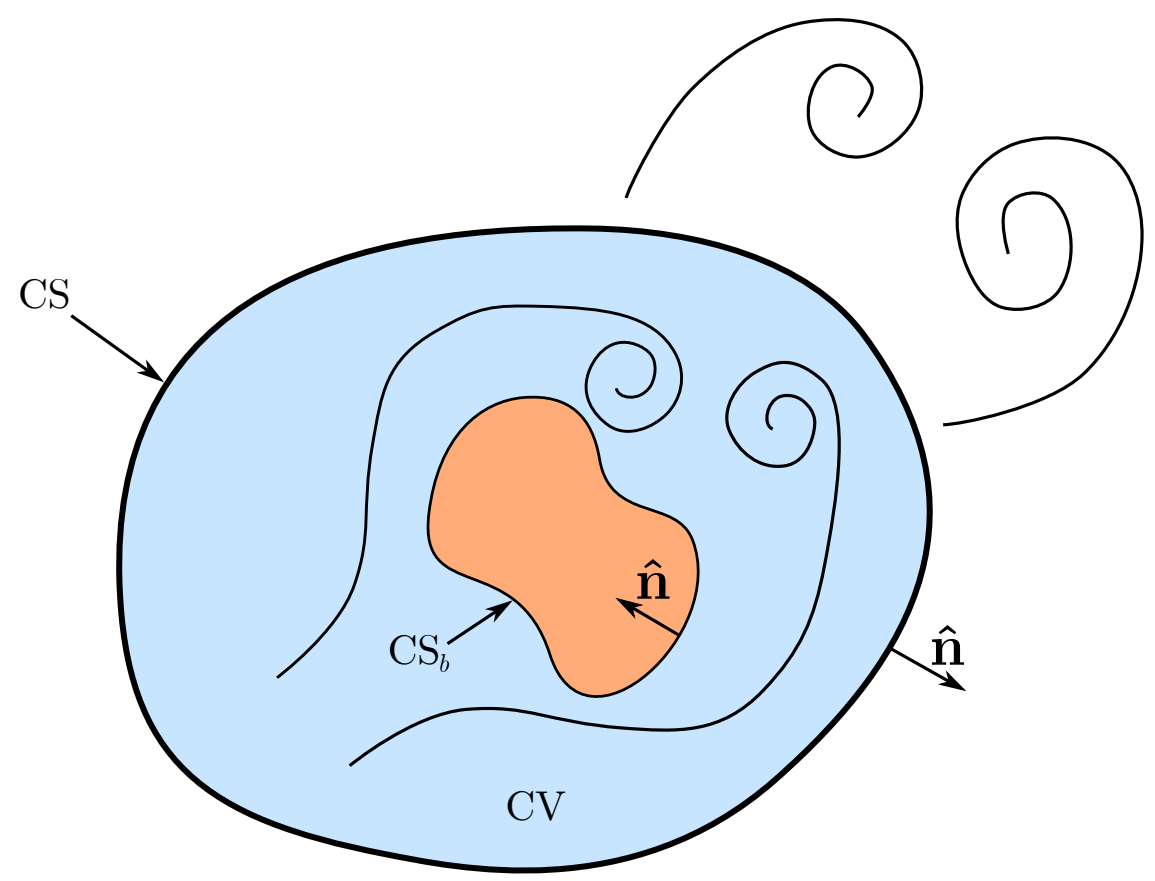

Figure 2.1: The domain of integration (blue) for the evaluation of fluid-dynamic forces on a body (orange) is illustrated. Volumetric quantities are integrated over the control volume $(\mathrm{CV})$ and surface quantities are integrated over the control surface (CS). The direction of normal vectors $\hat{\mathbf{n}}$ are shown for both the outer CS and the body control surface $\left(\mathrm{CS}_{b}\right)$.

volume, the Leibnitz integral theorem can be applied to the unsteady term:

$$
-\rho \iiint_{\mathrm{CV}} \frac{\partial \mathbf{u}}{\partial t} \mathrm{~d} V=-\rho \frac{\partial}{\partial t} \iiint_{\mathrm{CV}} \mathbf{u} \mathrm{d} V
$$

such that under the assumption of incompressibility, Equation 2.4 then reduces to:

$$
\mathbf{F}(t)=-\frac{\partial}{\partial t} \iiint_{C V} \rho \mathbf{u d} V
$$




\section{$2.3 \quad$ Added-Mass Force}

When a body moves through a real fluid at constant velocity, drag resists its motion. In an ideal fluid (having finite density, but zero viscosity) however, a body at constant velocity will experience no net drag, since inviscid flows have no pressure losses and no wall-shear forces. As the body moves, fluid is displaced from in front of the body to its rear, going around and not through the solid body. Hence, this body motion induces some motion of the ideal fluid, though it still experiences no net drag. This contradiction to the drag felt by a body submersed in a real fluid at constant velocity is known as d'Alembert's paradox, brought forth by d'Alembert (1752). Based on this inviscid flow argument, one would incorrectly assume that in order for a body to change velocity, no force is required other than that to accelerate the mass of the body. However, even in an inviscid fluid, in accelerating the body, fluid around it must also be accelerated. As discussed by Brennan (1982), work must be done to increase the kinetic energy of the fluid around a body as well as to increase the kinetic energy of the body itself. The force associated with the increase of kinetic energy of the surrounding fluid is termed the added-mass force, as there exists some fluid mass that is additional to the mass of the body that must also be accelerated with the body. The added mass $\left(m_{a}\right)$, as described by Batchelor (1967), is not defined in space but rather is calculated through an integration of the change in velocity of all fluid around the body.

It may be useful to understand the concept of added mass using the following analogy by Darwin (1953): A cart is going along a muddy road, and its wheel picks up mud. This mud is carried round for one turn of the wheel, and is then deposited on the ground again. Neither when picked up nor when set down does the mud receive 
A)

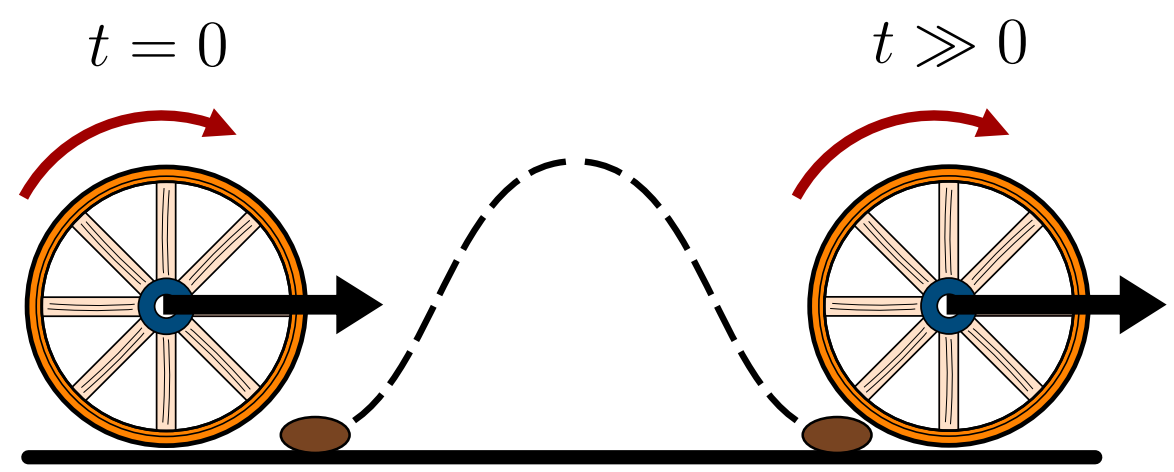

B)

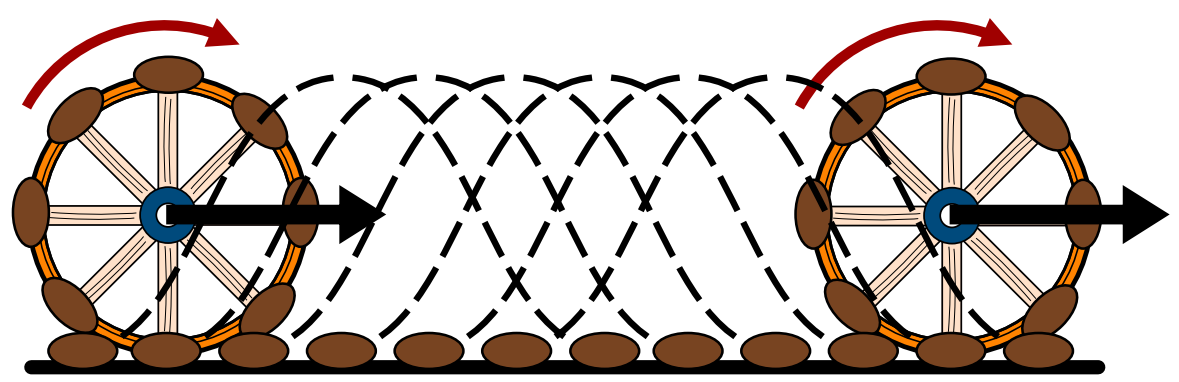

Figure 2.2: A) A wheel and mud illustration represents Darwin's added-mass analogy. A wheel picks up mud, representing the added mass, from the road and sets it back down after one rotation, changing only its displacement. B) A quasi-continuous system of mud on a wheel represents a closer analogy to the added-mass effects of a body moving through a fluid. Although the mud imparts no net force onto the wheel during constant velocity operation, the mud (added mass) influences the required force necessary to accelerate the wheel (submersed body).

any energy or momentum from the cart, nor does it give any to it. The only effect is that the mud is displaced to a new position farther forward along the road and set down there at rest. Yet evidently the mass of the mud on the wheel must be counted in estimating the forces needed to move the cart.

This analogy describes the long term effect of carrying mud on a wheel: the mud undergoes displacement but receives no net change in velocity. If this analogy is taken one step further, a quasi-continuous system is more representative of a fluid continuum and is visualized in Figure 2.2. 
Relating back to Equation 2.6, the total integration of the fluid's density is equal to some fluid mass. If we let this fluid mass be the nominal fluid mass affected by the change in the body's velocity $\mathbf{u}_{b}$, Equation 2.6 takes a simplified form:

$$
\mathbf{F}(t)=\frac{\partial}{\partial t}\left(m_{a} \mathbf{u}_{b}\right)
$$

It then follows that by expanding Equation 2.7 using the product rule, we arrive at the equation

$$
\mathbf{F}(t)=\frac{\partial m_{a}}{\partial t} \mathbf{u}_{b}+m_{a} \frac{\partial \mathbf{u}_{b}}{\partial t}
$$

which is the underlying equation used in the force estimation technique developed in Chapter 3.

The assumptions made in the simplification from Equation 2.4 to Equation 2.8 may not hold when applied to experimental data from a real fluid flow. For example, using a field of view that is not sufficiently large, where the external control surface is not everywhere ambient conditions, the surface integrals of Equation 2.4 must be taken into consideration. The pressure field propagates away from the body at the speed of sound in the medium, which in low Reynolds number flows is considerably faster than the body's velocity. Directly correlated to the near-instantaneous change in the pressure field is the convection of fluid particles, due to the existence of pressure gradients that accelerates the fluid, which in turn causes changes in the viscous stress tensor. These three terms on the control surface can only be neglected if their relative contributions are small compared to the unsteady term in the classical force formulation. The convective, pressure and viscous terms will not integrate to a negligibly small quantity if the external control surface is not sufficiently far away from 
the body.

The convective and viscous terms can be calculated directly from the velocity field, however calculation of the pressure term is indirect. Pressure can be extracted from three-dimensional flow data using a variety of methods, many of which are compared by Gent et al. (2017), and is a nontrivial undertaking on its own. Because of the difficulties associated with extracting pressure from optical flow field measurements, the force estimation method explored in this thesis relies on a control volume large enough for the integration of the pressure term to be assumed to be negligible.

More importantly, the assumption that allows for the jump from Equation 2.6 to Equation 2.7 is that the fluid that moves in phase with body (the fluid that represents the added mass) is the result of the volumetric integration of density in the flow field. This assumption can be expressed as the following:

$$
\frac{d}{d t}\left(\int_{C V} \mathbf{u} \rho d V\right)=\frac{d}{d t}\left(m_{a} \mathbf{u}_{b}\right) .
$$

Classically, the added mass of a body only exists when the body is undergoing an acceleration. The added mass is calculated as the volumetric integral of accelerating particles over all space. Equation 2.9 implies that the force calculated by Equation 2.8 holds during acceleratory motions, but may not be intended to hold for constant velocity motion. These assumptions suggest limitations of steady-state drag calculation, that is the time-averaged drag coefficient at constant velocity, when attempting to apply Equation 2.8.

To illustrate this mathematical limitation, let us consider an example of an infinitely large control volume. At constant velocity the flow conditions far upstream and far downstream of a body translating through a viscous fluid are quiescent. The 
fluid is at rest in the far field because viscous energy dissipation is bringing all flow to zero velocity. The drag force on the body is a byproduct of the energy balance between energy injected into the flow by surface interactions on the moving body, and energy dissipated from the flow by viscous heat generation. The energy lost to thermal heat generation is part of an irreversible process, whereby kinetic energy is irrecoverable. Conversely, Weymouth and Triantafyllou (2013) showed that the energy stored in the added mass can be recovered as negative drag (thrust) in the case of a decreasing frontal area, which is a reversible process. If the time-varying added-mass force is a result of a reversible process, and constant velocity drag is a result of an irreversible process, then it would be a physical contradiction to assume that one can give the other.

\subsubsection{Coefficient of Added Mass}

Classically, the added mass of an accelerating body is assumed to be constant, nullifying the time rate of change of added mass term in Equation 2.8. The fluid force due to acceleration, in this instance, then becomes solely a product of added mass and body acceleration. This version of the added-mass force was notably investigated by Daniel (1984), who used biological propulsion as the inspiration for research into added-mass coefficients of varying geometry bodies, shown in Figure 2.3. The addedmass coefficient $c_{\mathrm{ii}}$ is defined as the ratio of added mass to the displaced fluid mass (i.e. the volume occupied by the body $V_{b}$, multiplied by the fluid density $\rho$ ):

$$
c_{\mathrm{ii}}=\frac{m_{a}}{\rho V_{b}} .
$$




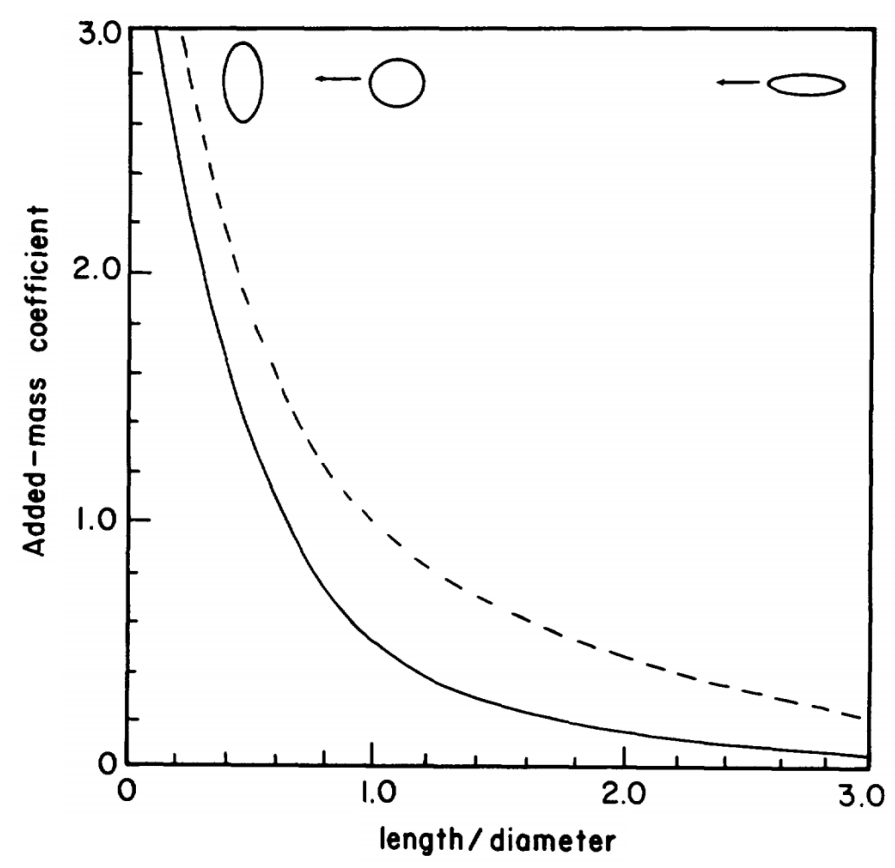

Figure 2.3: Added-mass coefficients are derived from potential flow theory for ellipsoids (solid line) and elliptical cylinders (dashed line) for varying length to diameter ratios. The length to diameter ratio is the ratio of the axis parallel to the direction of motion to the axis perpendicular to the direction of motion. Figure from Daniel (1984).

The added-mass coefficient of an accelerating body in potential flow is purely a function of its geometry. In a similar manner to Daniel (1984), the added-mass coefficients associated with bodies of varying geometries have been derived and documented by Brennan (1982). These added-mass coefficients can be used to calculate the addedmass force in accelerating potential flows as follows:

$$
\mathbf{F}=c_{\mathrm{ii}} \rho\left(\frac{D}{2}\right)^{3} a,
$$

where $\rho$ is the fluid density, $D$ is the hydraulic diameter of the body and $a$ is the acceleration of the body within the fluid. 


\subsubsection{Time-Varying Added Mass}

The idea of a varying added-mass force was investigated by Weymouth and Triantafyllou (2012) and Weymouth and Triantafyllou (2013) who rapidly reduced the frontal area of cylinders and ellipsoids to recover added-mass energy in the form of thrust. Another example of variable added mass was studied by Fernando and Rival (2017) who used a pitching plate to change the effective frontal area encountered by the free stream, while simultaneously accelerating, incurring a time-dependent added-mass force. Hence, the time rate of change of added mass in Equation 2.8 is non-zero and $m_{a}$ represents an instantaneous added mass rather than a single, constant, added mass.

The classical derivation of added mass involves a fixed geometry body undergoing constant acceleration, where the added mass is constant in time. Although these previous examples have demonstrated a changing added-mass force, they have done so by changing the effective frontal area of the geometry. A main idea investigated later in this thesis is that for constant acceleration of a fixed-geometry body, having no change in effective frontal area, the added mass of a fixed-geometry body can is non-constant, changing at each moment in time, and can be calculated using a driftvolume approach. This approach is fully detailed in Chapter 3 and provides the basis for the force estimation technique proposed in this thesis.

\subsection{Force Formulations from Vorticity}

Amongst the vortex dynamics described by Saffman (1992), a technique using vorticity in the flow exists to estimate fluid forces acting on a body. Vorticity $\omega$, the curl of the velocity field $\mathbf{u}$, is a measure of rotation in the flow and is related to the kinetic 
energy imparted to the fluid by the body:

$$
\omega=\nabla \times \mathbf{u} .
$$

Vorticity is only generated at fluid/surface boundaries and hence can be used to define the force acting on a body, equal and opposite to the force of the body acting on the fluid, as follows:

$$
\mathbf{F}=\rho \iiint_{\infty}(\mathbf{u} \times \omega) \mathrm{d} V .
$$

This expression requires that the vorticity everywhere in the flow be resolved, even within the boundary layer, which is a challenging task even with modern technology; Kähler et al. (2012) exposes the near-wall bias errors associated with current PIV technology. Because near-wall resolution and having a large FoV are competing requirements in terms of optical setup, Wu et al. (2006) developed a bounded control volume approach that deals with vorticity flux through a control surface. Their force estimation method incorporates the rate of vorticity production and the bounded vorticity as follows:

$$
\mathbf{F}=-\rho \frac{d \mathbf{H}}{d t}+\rho \iiint_{\mathbf{C V}}(\mathbf{u} \times \omega) \mathrm{d} V,
$$

where $\mathbf{H}$ is the hydrodynamic impulse and represents a mathematical proxy for the body surface vorticity contribution, such that

$$
\mathbf{H}=\frac{1}{2} \iiint_{\mathrm{CV}}(\mathbf{x} \times \omega) \mathrm{d} V .
$$

The second term in Equation 2.14 is commonly referred to as the vortex force or Lamb vector, and accounts for the wake development shedding off of the body surface in 
time. This method can be successful when applied to a large enough control volume; however, the dynamic spatial range of current measurement technology limits the ability to have both a large CV and sufficient near-wall resolution, especially at higher Reynolds numbers.

Other Eulerian force measurement techniques are reviewed by Rival and Oudheusden (2017), such as a derivative-moment transformation (DMT) method, presented by Wu et al. (2005) and a vorticity formulation, from Noca et al. (1997). The DMT method uses a version of Gauss' theorem to transform the volumetric integral of Equation 2.4 into a control surface integral. This transformation reduces the dependency on accurate acceleration values within the flow field, but has been shown by Mohebbian and Rival (2012) to introduce large sources of error when wake-vortices pass through the control surface. While this method bypasses the need for high quality data near the wall, errors still exist when high spatial velocity gradients are present on the control surface. The vorticity formulation of Noca et al. (1997), due to its many gradient operations, derivatives and multiplications, typically produces significantly larger error comparatively, as explained by Noca et al. (1999).

In summary, the main caveat of the existing Eulerian force estimation techniques is that, for insufficient resolution in key regions around the body and on the control surface, error production is rampant through the many spatial and temporal operators necessary for force output. Table 2.1 shows an overview of error propagation sources associated with the mathematical operations required to fully compute fluid force in an Eulerian frame of reference. Multiplications, temporal derivatives and spatial derivatives each contribute to error propagation for measurements with experimental uncertainty. One of the objectives of the new Lagrangian force estimation technique 


\subsection{ROTATIONAL AND IRROTATIONAL FORCE}

Table 2.1: Overview of error sources for the examined Eulerian force-extraction techniques. Each multiplication, temporal derivative and spatial derivative propagates errors throughout the calculation. Table adapted from Rival and Oudheusden (2017).

\begin{tabular}{lllll}
\hline Method & No. terms & $\begin{array}{l}\text { No. mul- } \\
\text { tiplica- } \\
\text { tions }\end{array}$ & $\begin{array}{l}\text { No. tem- } \\
\text { poral } \\
\text { deriva- } \\
\text { tives }\end{array}$ & $\begin{array}{l}\text { No. } \\
\text { spatial } \\
\text { deriva- } \\
\text { tives }\end{array}$ \\
\hline \hline $\begin{array}{l}\text { Classical formulation } \\
\begin{array}{l}\text { Derivative-moment } \\
\text { transformation method }\end{array}\end{array}$ & 4 & 2 & 2 & 3 \\
\begin{tabular}{l} 
Vorticity formulation \\
\hline
\end{tabular} & 9 & 3 & 2 & 3 \\
\hline
\end{tabular}

is to simplify the operations required for force calculation in order to reduce error propagation.

\subsection{Rotational and Irrotational Force Decomposition}

The forces that arise when fluid accelerates around a body have classically been resolved into added-mass, viscous-drag, and flow-history contributions, much of which is described by Odar and Hamilton (1964), Karanfilian and Kotas (1978), and Clift et al. (1978). Classical aerodynamic models proposed by Theodorsen (1935) and Küssner (1936) have elicited force decompositions involving circulatory and non-circulatory (added-mass) effects. A few recent studies, such as Baik et al. (2012), have applied this type of decomposition to the force histories of accelerating airfoils in separated flows.

The non-circulatory lift on an impulsively started flat plate at an angle of attack of $15^{\circ}$ was predicted by Pitt Ford and Babinsky (2013) using a complex potential-flow 
solution using circulation from measurements. They showed that, during the acceleration period, the added-mass force was responsible for nearly half the total lift on the body. The remaining lift force was attributed to circulation in vortices detached from the plate, rather than bound circulation. Mancini et al. (2015) performed a similar analysis on accelerating flat plates at varying angles of attack using the solution for the added mass derived by Pitt Ford and Babinsky (2013) to determine the relative contributions of both the circulatory and non-circulatory effects.

Other investigations by Manar et al. (2016) and Babinsky et al. (2016) have utilized the two-part force decomposition for accelerations from rest. Their analyses also focus on the lift generated from accelerating wings where they showed that a simple low-order decomposition of circulatory and non-circulatory forces was able to predict the total wing loading.

\subsection{Lagrangian Force Estimation Techniques}

There exists inherent temporal information in particle pathlines reconstructed from LPT techniques, such as the Shake-The-Box algorithm developed by Schanz et al. (2016), which triangulates particles in three-dimensional space and fits them onto smooth pathlines developed over time. Time-resolved force estimation can contain fewer derivative errors using Lagrangian data as a starting point and can produce alternative measurement methods if the history of fluid particle positions is used.

Vortex added mass was calculated by Dabiri (2005) based on the concept of a drift volume, a Lagrangian tool originally developed by Darwin (1953), which is discussed in the following section. The expression developed by Dabiri (2005) to predict the 
instantaneous vortex force is as follows:

$$
\mathbf{F}(t)=\rho \frac{\partial}{\partial t} \iiint_{C V}(\mathbf{x} \times \omega) \mathrm{d} V+\rho c_{i i} \frac{\partial}{\partial t}\left(\Omega_{V} \mathbf{u}_{V i}\right)
$$

where the first term is similar to the hydrodynamic impulse term seen in Equation 2.14, but the second term, the wake-vortex added mass, contains an added-mass coefficient $c_{i i}$, a wake-vortex volume $\Omega_{V}$, and a wake-vortex velocity $\mathbf{u}_{V i}$ relative to the body in the $\mathrm{i}^{\text {th }}$ direction. The first term, being similar to the hydrodynamic impulse term seen in vorticity formulations, propagates many experimental errors due to the calculation of successive spatial and temporal derivatives.

While Dabiri (2005) did not initially measure Lagrangian quantities, synthetic particles were seeded into the Eulerian vector field, captured by planar PIV, where their positions were interpolated forwards in time. This Lagrangian data extraction method is used extensively later within this thesis. In Equation 2.16, the addedmass coefficient is affiliated with a fluid vortex, rather than a solid body, and is a constant value, appropriate for application to constant velocity motion. This addedmass coefficient, as Equation 2.10 suggests, is calculated from a known added mass associated with the translating object. The method by which this added mass is calculated is derived from a drift volume and is the topic of the following section.

\subsection{Drift Volume and Darwin's Proposition}

Added mass was proposed by Darwin (1953) to be a calculable measurement, even in potential flow, using a drift-volume approach. A drift volume $\Omega_{d}$ is defined as an integration of the total downstream displacement that Lagrangian fluid particles 


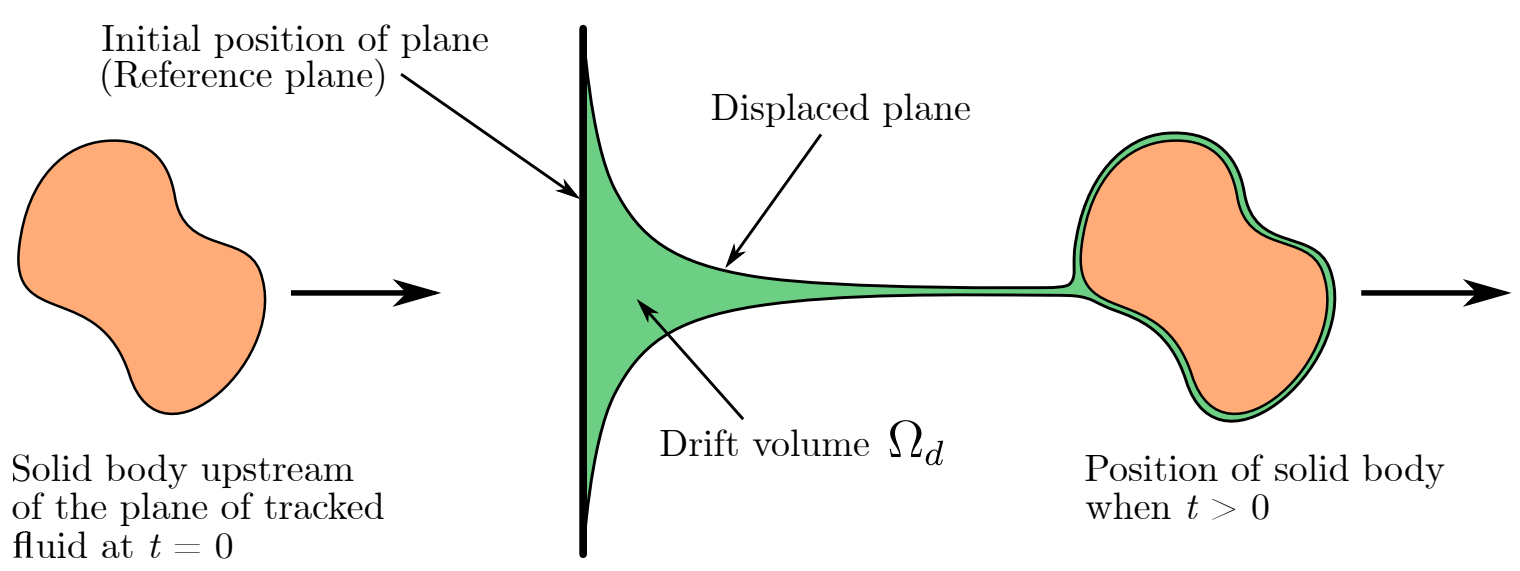

Figure 2.4: Illustration of a drift volume, as defined by Darwin (1953). A body, initially upstream of a reference plane of tracked fluid, passes through the plane of fluid, bending it around the body. Fluid initially upstream of the reference plane undergoes a net downstream displacement, known as a drift volume (green).

undergo when a body passes through a tracked plane of fluid, illustrated in Figure 2.4 .

To further understand the concept of a drift volume, the potential flow solution for a constant velocity sphere can be used as an example. The velocity components for potential flow around a sphere are calculated by the following equations:

$$
\begin{gathered}
U_{x}(x, y, z)=U_{\infty}\left(1-\frac{3 R^{3} x^{2}}{2 r^{5}}+\frac{R^{3}}{2 r^{3}}\right) \\
U_{y}(x, y, z)=-U_{\infty} \frac{3 R^{3} x y}{2 r^{5}} \\
U_{z}(x, y, z)=-U_{\infty} \frac{3 R^{3} x z}{2 r^{5}}
\end{gathered}
$$

where $x, y$, and $z$ are the local Cartesian coordinates, $U_{x}, U_{y}$, and $U_{z}$ are the local Cartesian velocity components, $U_{\infty}$ is the free stream velocity, $R$ is the radius of the sphere, and $r$ is the distance from the axis of symmetry, equal to the root sum squared 
of $y$ and $z$ :

$$
r=\sqrt{y^{2}+z^{2}} .
$$

Streamlines are a visualization tool used to analyze the movement of fluid. Because they are instantaneously and everywhere tangent to the velocity field, they show the direction in which a massless fluid element will travel at any point in time. The streamlines around a sphere, shown in Figure 2.5, are calculated directly from the velocity field, requiring no knowledge of the motion of individual fluid particles. The spacing between streamlines dictates the velocity, as one of the properties of streamlines is that the mass flux between neighboring streamlines remains constant in steady flow. Hence, the narrowing gaps between streamlines near the spheres sides produces higher velocities, seen in Figure 2.6. These higher velocities produce reverse flow relative to the sphere's motion when viewed from a reference frame of the ambient, stationary fluid.

If synthetic fluid particles are seeded into the flow and allowed to propagate with the flow, Lagrangian pathlines are generated, seen in Figure 2.7. For a given plane of tracked fluid particles, the drift volume is the region between the final position of particles and their original position. The drift-volume region in Figure 2.7 is identifiable as a conical region, just as Figure 2.4 illustrated.

The net downstream displacement of fluid mass is theorized by Darwin (1953) to be equal to the added mass carried by a moving body:

$$
m_{a}=\rho \Omega_{d}
$$




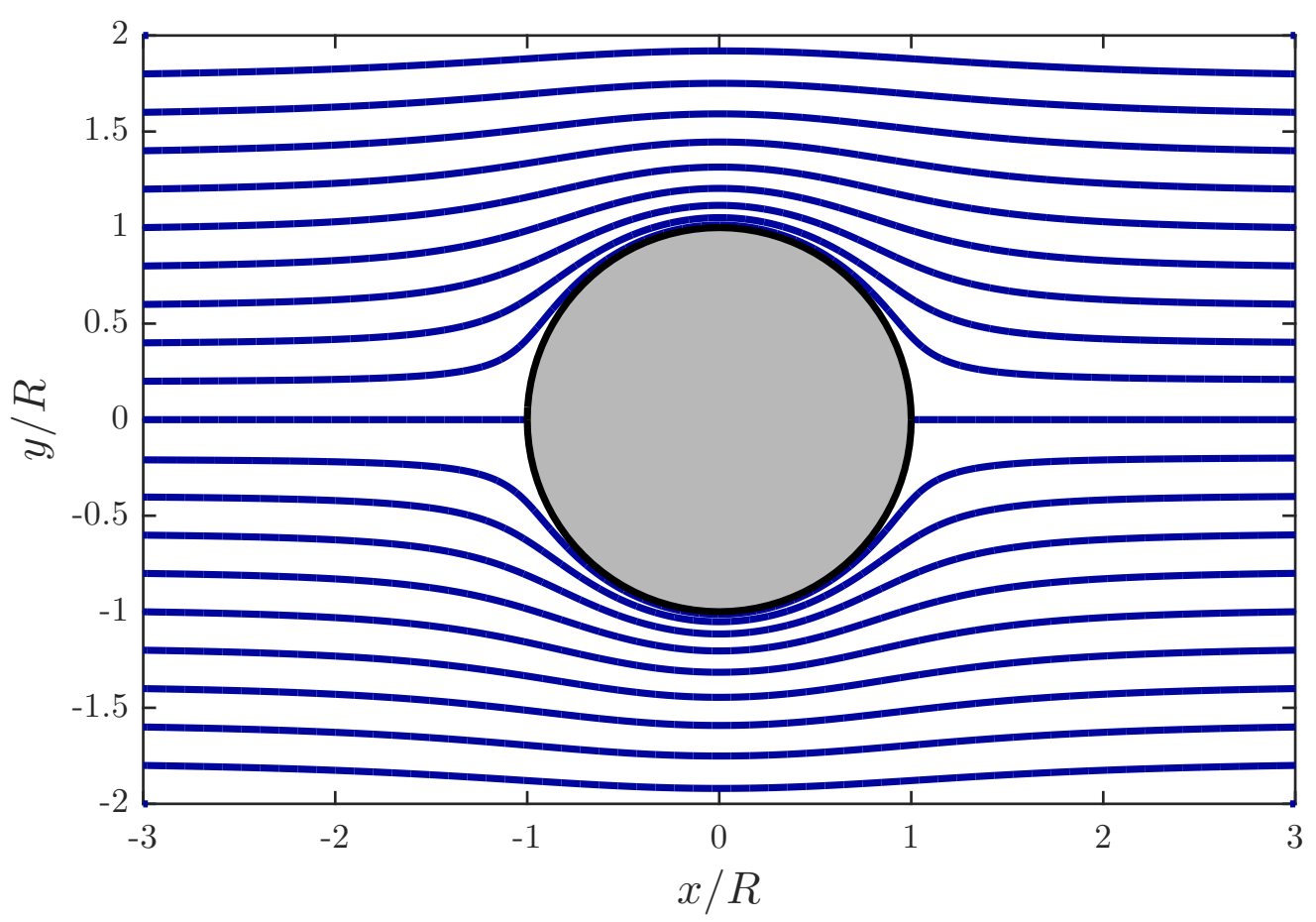

Figure 2.5: Potential flow streamlines around a sphere in steady flow. Viewed from a reference frame attached to a sphere at constant velocity, the movement of fluid around the sphere is described by stationary streamlines. The narrowing gaps between streamlines around $y / R= \pm 1$ indicate higher streamwise velocity, producing fluid that has a velocity magnitude larger than that of the translating sphere, but in the opposite direction. This phenomenon is better visualized in Figure 2.6.

This drift-volume, added-mass equivalence has has been validated on several occasions: Darwin's proposition is an appealing result, due to its relative simplicity, and therefore was further investigated by many others, including Lighthill (1956), Yih (1985), and Benjamin (1986). Analytical assessments of drift volumes on a constantvelocity potential-flow sphere were performed by Darwin (1953) and Eames et al. 


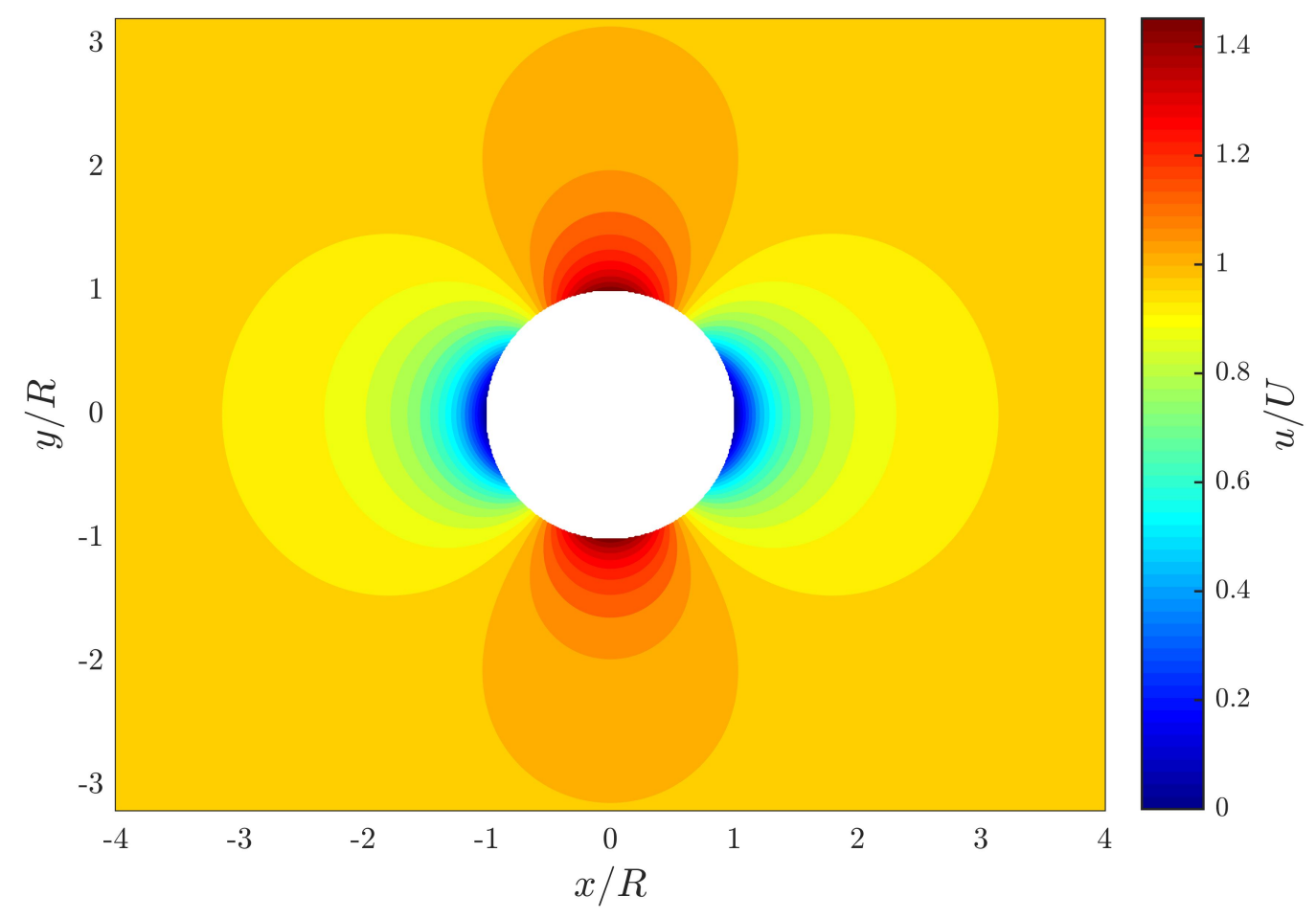

Figure 2.6: Contours of $x$-direction velocity are shown for the potential flow solution of a sphere translating in steady flow. The flow moves from left to right around a stationary sphere (white). In the blue regions, flow is slowed down relative to the sphere, bringing the fluid closer to rest. In red regions, fluid is accelerated around the sides of the sphere to a velocity greater than the free stream velocity. This downstream acceleration of fluid suggests that flow reversal occurs if viewed from a stationary (ambient) frame of reference. Particle pathlines in Figure 2.7 show these flow reversal events clearly. 


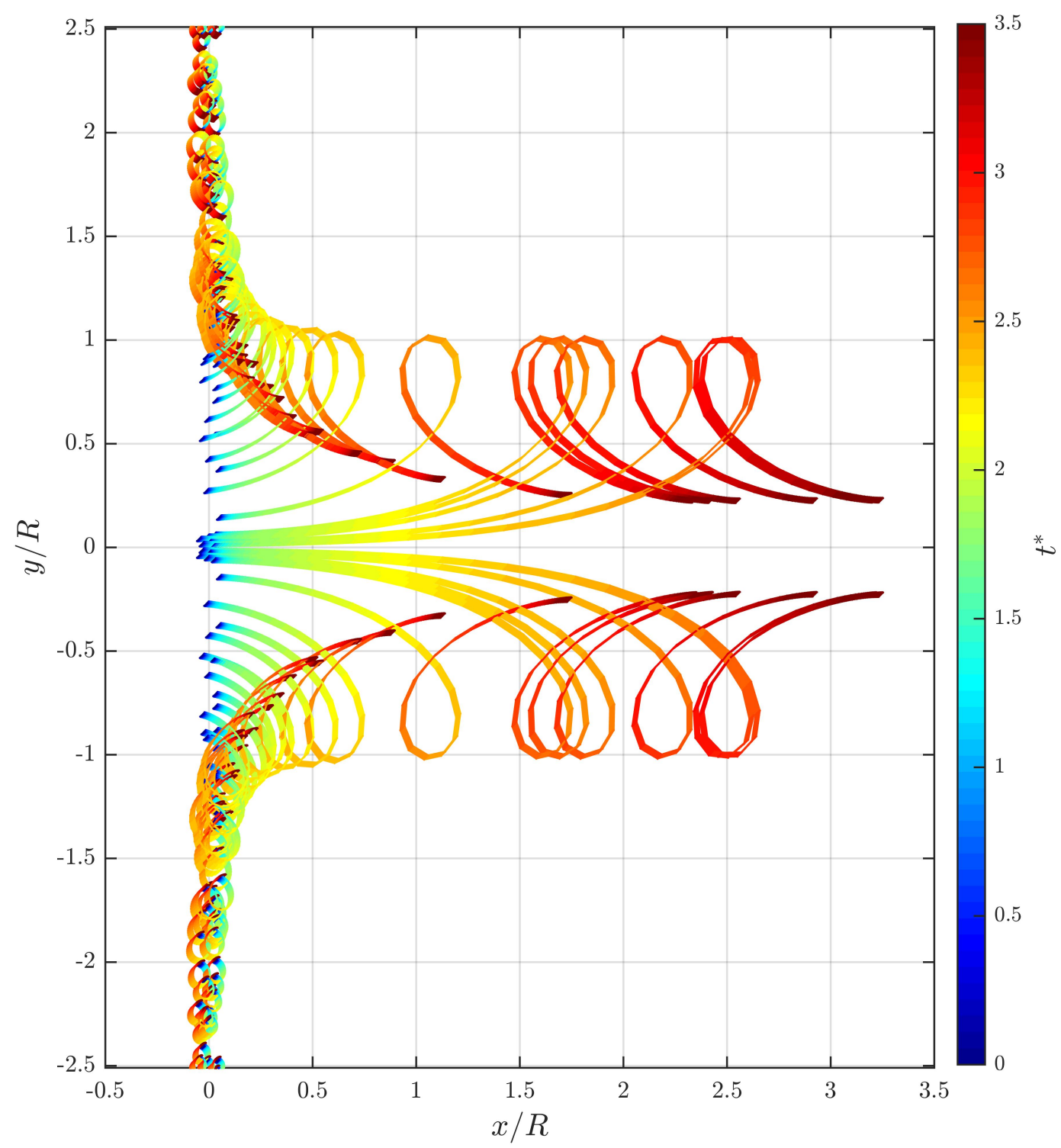

Figure 2.7: Particle pathlines, coloured by time, are shown for the potential flow solution of a constant velocity sphere traveling in the $+x$-direction. Each pathline originates from the $x / R=0$ plane (within a tolerance of $|x / R| \leq$ 0.05 ), and is convected downstream. Flow reversal events, called elasticas, form loops in the particle pathlines due to the regions of increased relative velocity around the sides of the sphere. The drift volume associated with a potential flow sphere is viewable as the region between particles at $t=0$ (blue) and $t \gg 0$ (red). 
(1994), whereas Dabiri (2005) was able to calculate the added-mass coefficient of a translating fluid vortex by means of a drift volume developed from a wake vortex.

More importantly, Equation 2.21 alludes to the existence of a non-constant added mass, since drift volume development has an associated time-scale, which is not instantaneous. Hence, the proposition that added mass can change with time allows for the added-mass force to be characterized in a non-constant way, just as in Equation 2.8 .

While these first Lagrangian force estimation results are quite promising, the quandary of time-resolved force estimates in separated flows persists. The method of unsteady force estimation developed in the next chapter of this thesis relies heavily on the time-varying aspect of added mass and its associated growth rate to estimate the fluid forces experienced by accelerating objects. 


\section{Chapter 3}

\section{Proposed Force Estimation Technique}

This chapter provides a description of the experimental test case used to validate the novel unsteady force estimation technique. It details the limitations of drift-volume calculations from a single reference plane, and it explains a new multi-plane driftvolume calculation method for the purpose of measuring time-varying added mass.

\subsection{Description of Test Case}

Until now, added-mass calculations from drift volumes developed from solid bodies have only been investigated using potential flow analysis. In order to apply this area of study to real, viscous fluid flows, a first experimental test case was considered. Of particular interest is an accelerating case, where added-mass effects contributing to larger forces can be measured. A circular flat plate, accelerated normal to the flow, is chosen as the first test case for the force estimation technique in question. For sufficiently high Reynolds numbers, the circular flat-plate geometry is dominated by flow separation, providing a test case that is dissimilar from the potential flow solution. In order to choose an appropriate acceleration magnitude, a variety of 


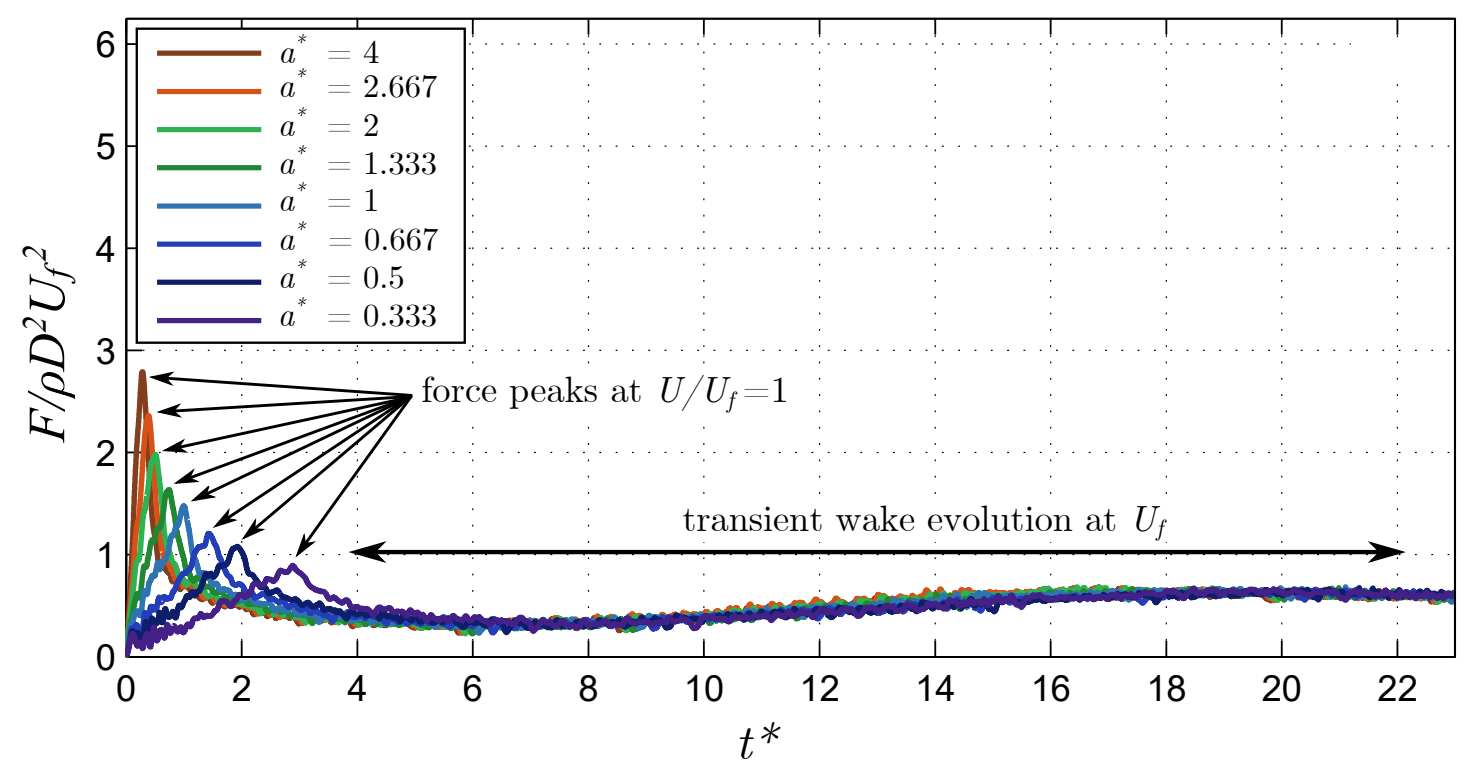

Figure 3.1: Force versus acceleration modulus showing varying peak force. Each curve represents an accelerating case where a circular flat plate starts from rest and accelerates to the final towing velocity $U_{f}$, but over varying time intervals. The relationship between acceleration modulus $a^{*}$ and acceleration time $t_{a}^{*}$ is inversely proportional: $t_{a}^{*}=1 / a^{*}$. Figure adapted from Fernando (2017).

acceleration moduli were investigated. Acceleration modulus $a^{*}$ is defined as:

$$
a^{*}=\frac{a D}{U_{f}^{2}},
$$

where $a$ is the dimensional acceleration, $D$ is the characteristic length scale, which here is the plate's diameter, and $U_{f}$ is the final towing velocity of the motion.

Figure 3.1 shows forces for 8 different values of $a^{*}$, where the most impulsive acceleration $a^{*}=4$ has the most defined added-mass force peak, and the slowest acceleration $a^{*}=0.333$ has the least defined peak. Within the limitations of the experimental apparatus used, the most impulsive acceleration modulus was chosen 

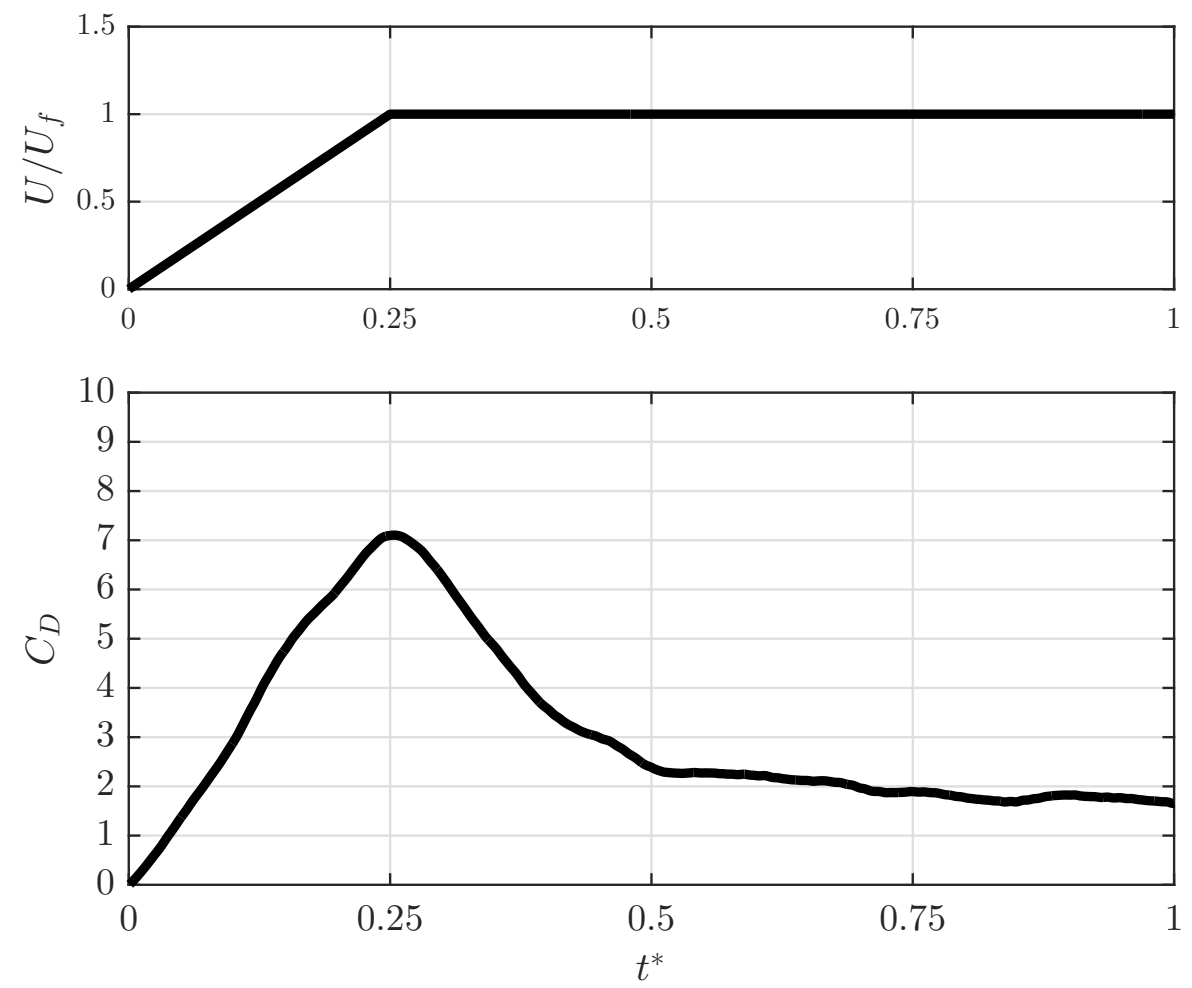

Figure 3.2: (top) The velocity profile of the accelerating plate is prescribed over time. Initially at rest, the plate undergoes constant acceleration at $a^{*}=4$ until the final towing velocity $U_{f}$ is reached at $t^{*}=0.25$, where it then maintains constant velocity. (bottom) The drag coefficient is measured using a physical force sensor. The plate's acceleration produces a force peak at $t^{*}=0.25$ due to added-mass effects.

for the test case as it contains a flow field which is least similar to potential flow, still produces a start-up vortex from flow separation, and has an easily discernible added-mass force peak. This middle ground between zero and infinite acceleration provides a test case that contains vortical structures in a highly separated flow, the most interesting fluid physics worth studying.

The kinematics of the plate's motion, shown in Figure 3.2, are such that the plate starts from rest, undergoes constant acceleration up to a final towing velocity $U_{f}$, and then travels at a constant velocity until steady-state conditions are reached. 
The normalized drag force, the coefficient of drag $C_{D}$, associated with this motion is defined by:

$$
C_{D}=\frac{F}{\frac{1}{2} \rho U_{f}^{2} A},
$$

where $F$ is the measured force, $\rho$ is the fluid density, $U_{f}$ is the final towing velocity and $A$ is the plate's frontal area. The measured drag is recorded from a physical force sensor attached to the plate and is also shown in Figure 3.2. Now that the physical description of the experimental test case is known, the drift-volume calculation method can be better understood.

\subsection{Single-Plane Drift-Volume Calculation}

Figure 3.3 shows pathlines, coloured by time, of fluid particles that start upstream of a reference plane placed at $x / D=0$ and are dragged downstream by the plate's translation in the $-x$ direction. From these tracks, the three-dimensional drift volume of the circular flat plate is calculated under the assumptions of axisymmetric flow. These assumptions are validated by Fernando and Rival (2016), who saw that the vortex ring developed in the wake of a circular flat plate undergoes stable, axisymmetric growth until $x / D=6$, at which point azimuthal instabilities and vortex detachment occur.

The drift volume is calculated from Lagrangian particle tracks calculated either from LPT or from synthetic seeding of velocity fields from PIV. In the current case, the assumption of axisymmetry allowed for a two-dimensional slice of PIV data along the plate's midspan to be representative of the entire flow field. Each particle was assigned a finite two-dimensional area equal to the total area in the field of view (FoV) divided by the total number of particles initially seeded. Particles were tagged either 


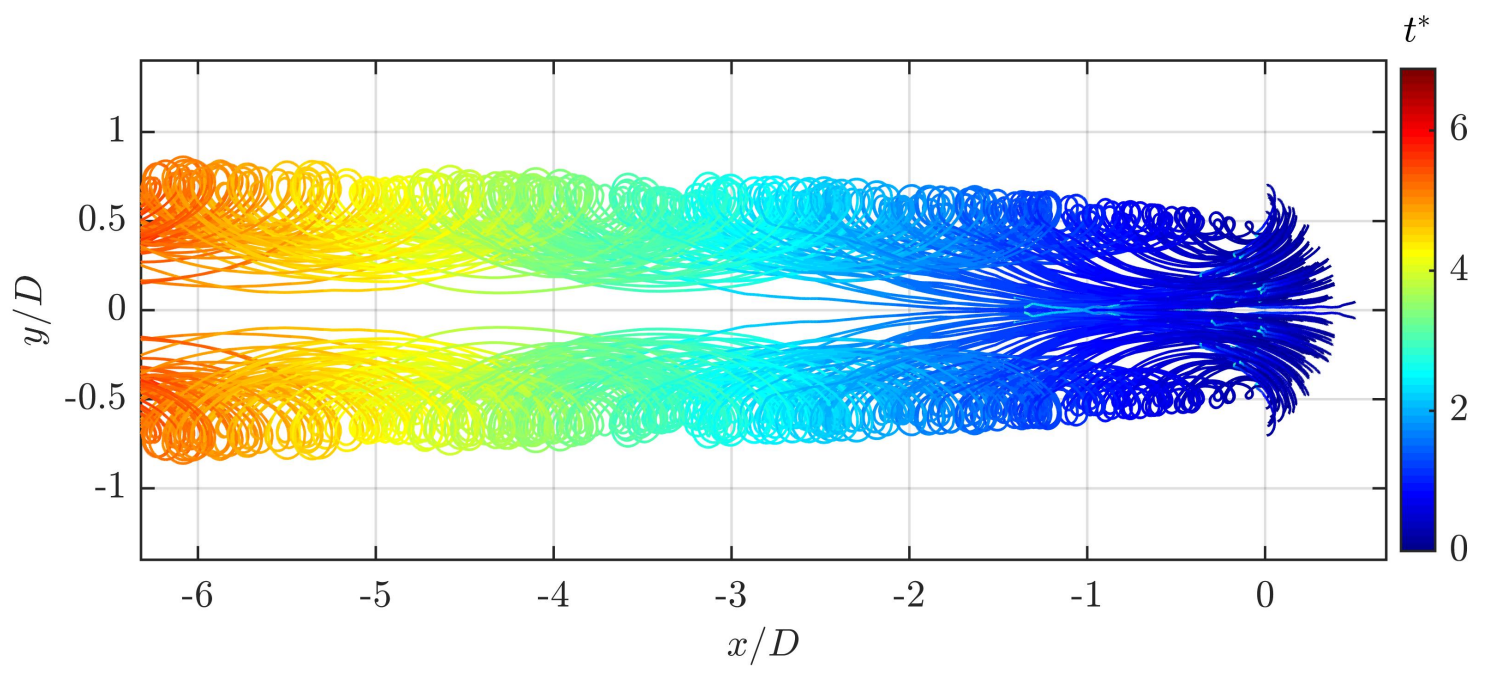

Figure 3.3: Pathlines are shown for synthetically seeded particles that originate upstream $(+x)$ of a reference plane placed at $x / D=0$. Tracks are shown only for particles that cross the reference plane, traveling from upstream of the reference plane $(+x)$ to downstream of the reference plane $(-x)$ when $t \gg 0$. For enhanced clarity, only $10 \%$ of tracks are shown.

as positive or negative contributors to the net drift volume, based on their direction of travel across the reference plane. Positively tagged particles (green in Figures 3.4 and 5.1) are those that originate upstream of the reference plane at $t^{*}=0$ and cross the reference plane to a downstream location when $t^{*}>0$. Negatively tagged particles (red in Figures 3.4 and 5.1) are those that originate downstream of the reference plane at $t^{*}=0$ and cross the reference plane to an upstream location when $t^{*}>0$.

The drift volume was calculated by performing a volumetric integral of revolution around the plate's circumference for each tagged particle in the two-dimensional FoV. A single reference plane, as used by Darwin (1953) and Eames et al. (1994) in their analysis of a sphere in potential flow, when used to compute the drift volume of 
continuous constant velocity motion, provides accurate calculations of added mass. Because there is no start or end time for a body traveling at constant velocity, the time scale at which the drift volume develops is irrelevant.

Because the added-mass force is in phase with the acceleration of a body, the calculation of added mass, and hence the drift volume, needs to be time-resolved. A drift volume developed from a single reference plane, regardless of its relative position to the body, is not representative of the time-resolved added mass due to its independent and uncorrelated time scale.

An example of reference plane selection and its associated drift-volume development is exemplified in Figure 3.4 where four different $x / D$ locations are chosen for a single reference plane to show their corresponding drift-volume development differences. In this figure, the plate accelerates from rest from the same position in each case, but the reference plane location is changed, resulting in different drift volumes. Due to the arbitrary nature of selecting an appropriate location for a single reference plane as well as the disparity between rapid acceleration and drift-volume time scales, a new multi-plane drift-volume calculation method was developed.

\subsection{Multi-Plane Drift-Volume Calculation}

A multi-plane drift-volume technique brings forth a solution to the problems associated with calculating added mass from single reference plane drift volumes. In the current experiment, a drift volume developed from a single reference plane placed at $x / D=0$, the start position of the plate, converges only after the plate has traveled $\sim 4 D$ downstream, whereas the acceleration takes place over a distance of $x / D=1 / 8$. 

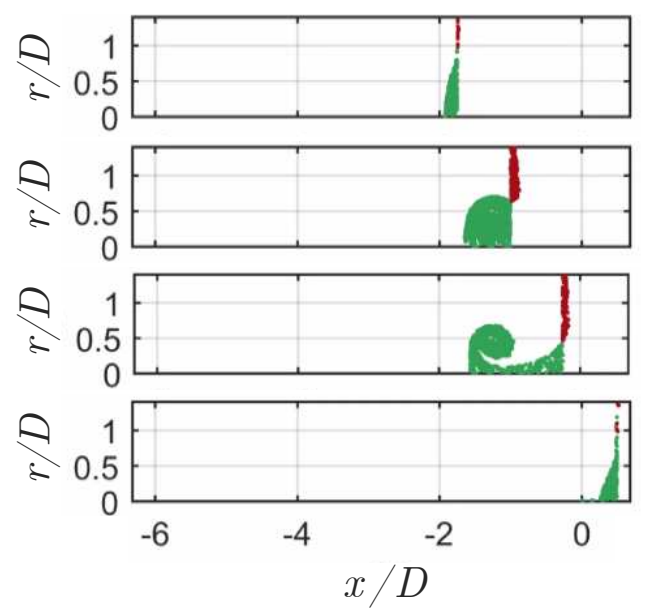

Figure 3.4: Drift-volume development is shown for four arbitrary chosen reference planes, equally spaced from $x / D=-1.75$ to $x / D=0.5$ at $t^{*}=2$. The amount of green (positive drift) fluid is noticeably different depending on reference plane location. In each case, the plate's position is $x / D \approx-1.5$.

The motivation for developing the multi-plane approach was to capture the instantaneous forces associated with the motion of all affected fluid around the body, not just the fluid that is able to pass through an arbitrarily-placed single reference plane. In this method, multiple reference planes are spaced equally apart, spanning the length of the FoV in the streamwise direction. The number of reference planes $N$ used in the drift-volume calculation was increased, decreasing the spacing between planes, until the noise level of the particle positions was approximately one order of magnitude lower than the distance between consecutive reference planes. The total drift volume $\Omega_{d}$ is calculated as the sum of the drift volume developed from each of the individual reference planes $\Omega_{d_{n}}$ :

$$
\Omega_{d}=\sum_{n=1}^{N} \Omega_{d_{n}},
$$

and is visualized in Figure 3.5.

The multi-plane drift-volume method requires special attention to be implemented 


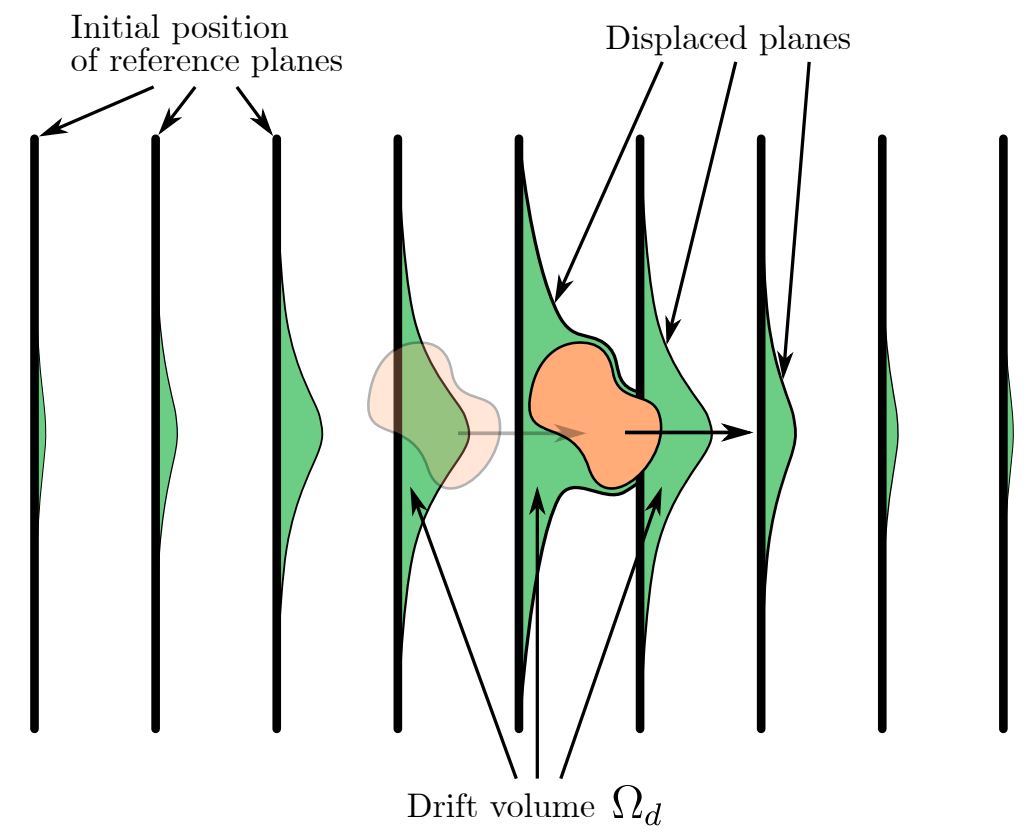

Figure 3.5: Illustration of multi-plane drift-volume calculation. The total drift volume $\Omega_{d}$ is the sum of the individual drift-volume contributions $\Omega_{d_{n}}$ from many single reference planes.

properly. If the simple case of $N=2$ reference planes is examined more closely, potential calculation artifacts may arise. When a body passes through the reference planes, a drift volume develops from each plane independently. A problem arises when a drift volume originating from the first reference plane propagates far enough downstream that it crosses through the second reference plane. As shown in Figure 3.6, a single fluid particle can end up being counted twice, once for each drift volume, if proper preventative measures are not first taken. In this instance, a region of overlap between two successive drift volumes exists and a double accounting of drift occurs. The same fluid particles are being counted once in the drift volume of the upstream plane and again in the drift volume of the next plane downstream. To avoid multiple accounts of a single Lagrangian fluid particle, a condition is enforced: In a multi-plane array, the drift volume extending from a single reference plane is 

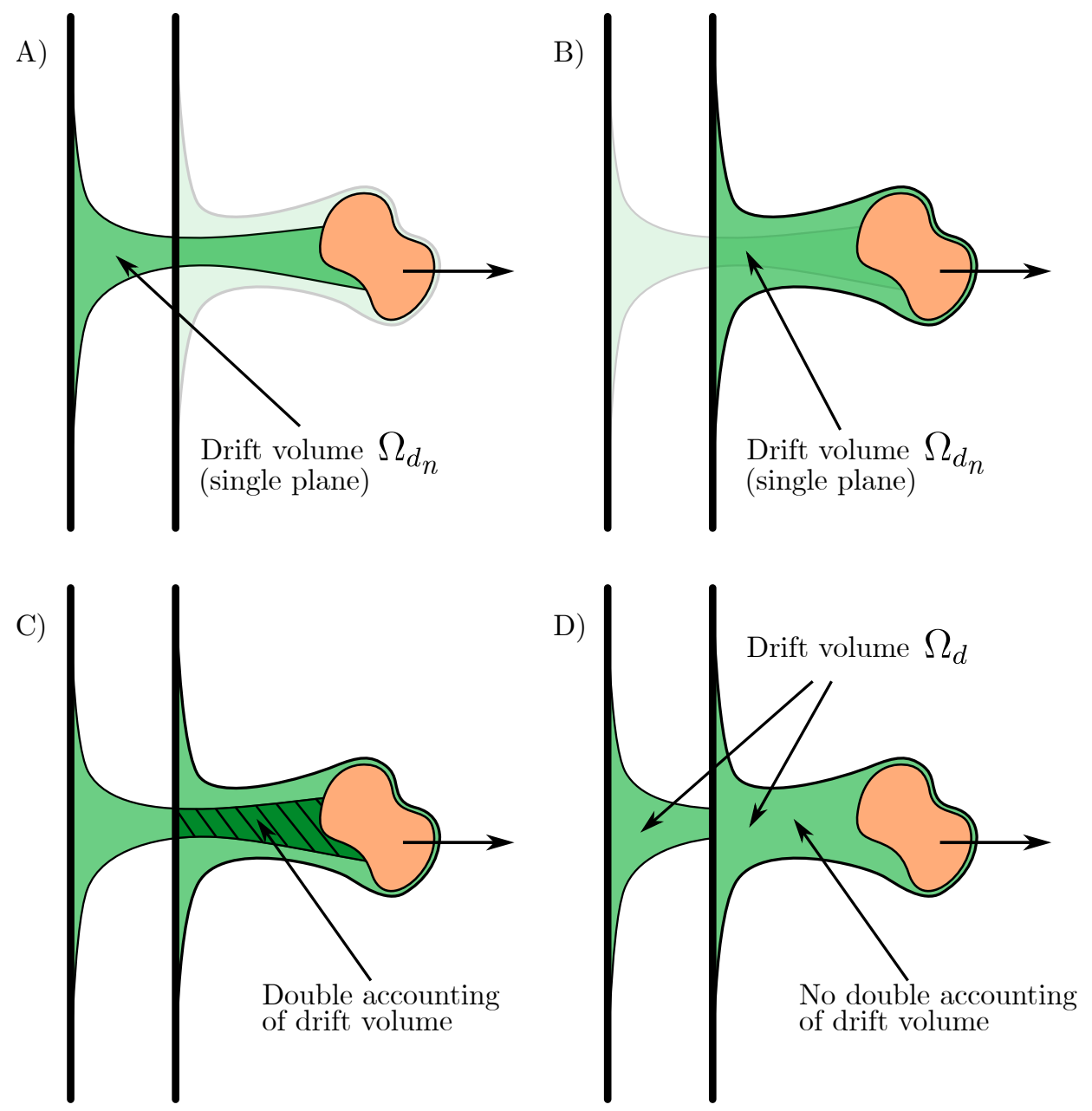

Figure 3.6: Double accounting of drift volume can occur when the drift volumes developed from two successive reference planes overlap. A) and B) show individual drift volume contributions $\Omega_{d_{n}}$ to the total drift volume $\Omega_{d}$. C) shows the overlapping region within which double accounting can occur. D) shows the rectified total drift volume $\Omega_{d}$, limiting the calculation of the drift volume in A) to within the region bounded by its neighbouring reference plane.

only calculated for the region bounded by its immediately neighbouring reference planes. Figure 3.6 depicts this overlapping drift region developed from two successive reference planes in a multi-plane array and includes a visual representation of how to calculate the multi-plane drift volume. 


\section{Chapter 4}

\section{Experimental Methods}

The following chapter provides a description of the test case used for validation of the force estimation method, the techniques that were used in the measurements, and the facility where the measurements were performed.

\subsection{Force measurements}

The accelerating flat plate experiment was performed in the towing-tank facility at Queen's University, shown in Figure 4.1, which is water-filled, has a square crosssectional area of $1 \mathrm{~m}^{2}$ and a total length of $15 \mathrm{~m}$. The towing tank is equipped with a roof that runs along the entire length of the apparatus to minimize free-surface effects. The plate underwent a motion consisting of a constant acceleration from rest to a final towing velocity over a distance of one eighth of a diameter. The motion was normalized using the following relationship for dimensionless time $t^{*}$ :

$$
t^{*}=t \frac{U_{f}}{D}
$$




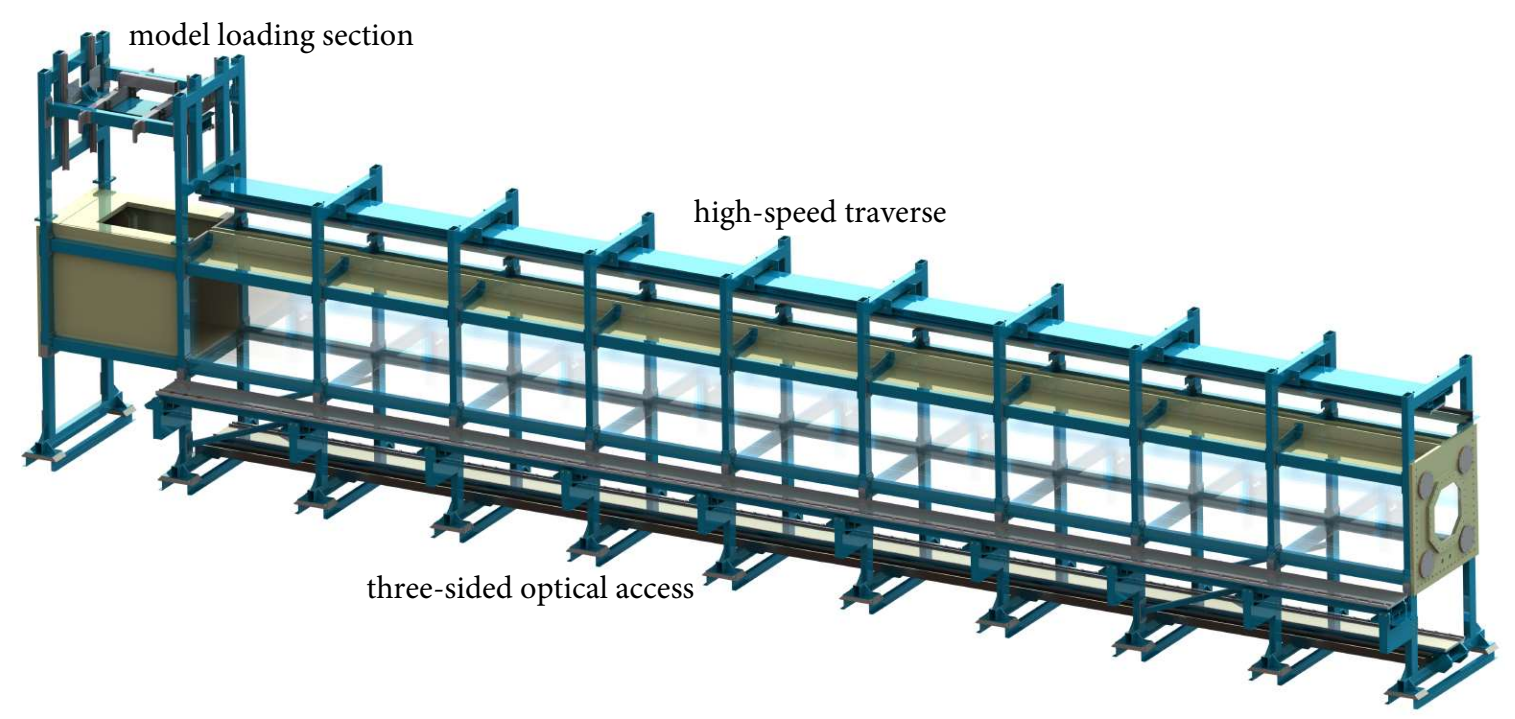

Figure 4.1: The towing-tank facility in the OTTER Lab at Queen's University measures $15 \mathrm{~m}$ in length, has a $1 \mathrm{~m} \times 1 \mathrm{~m}$ cross-section, and contains a slotted roof with a high-speed traverse system.

where $t$ is the physical time duration, $U_{f}$ is the final towing velocity, and $D$ is the plate's diameter. The plate was towed normal to the flow from rest to a final Reynolds number of $\operatorname{Re}=125,000$. The Reynolds number was calculated using the terminal velocity of the plate and its diameter:

$$
\operatorname{Re}=\frac{\rho U_{f} D}{\mu},
$$

where $\rho$ is the fluid density and $\mu$ is the fluid's dynamic viscosity. The plate was mounted perpendicular to a horizontal cylindrical sting, which had a diameter of $0.08 D$ and a nominal length of $2 D$. A section of the towing tank, approximately 3 $\mathrm{m}$ in length, is depicted in Figure 4.2, along with the mounting configuration of the plate. A six-component, submersible ATI Nano force transducer was mounted on the leeward side of the plate to record force data, and is depicted in the inset of Figure 


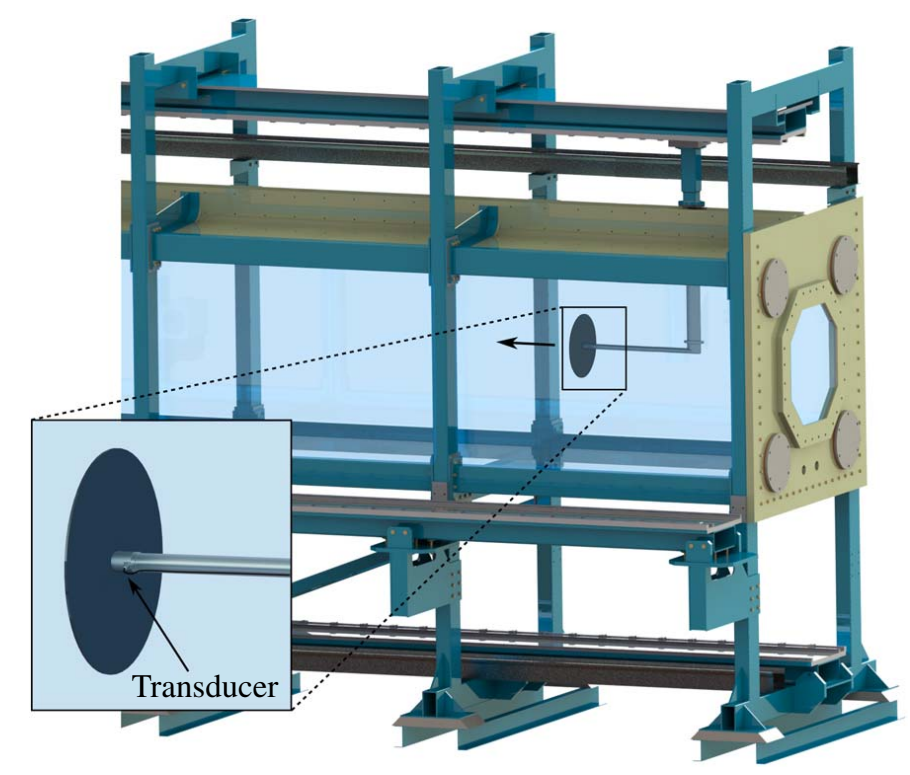

Figure 4.2: Towing-tank facility depicting the circular flat plate within the tank, and the location of the force transducer (shown in the inset).

4.2. The transducer had a static resolution of $0.125 \mathrm{~N}$ and was operated at a sampling rate of $1000 \mathrm{~Hz}$. The force data, shown in Figure 3.2 and again later in Chapter 5, was averaged over 20 runs.

\subsection{Particle image velocimetry measurements}

Time-resolved planar PIV was used to measure the time-dependent velocity field in the wake of the translating plate. The water-filled towing tank was seeded with nominally $100 \mu \mathrm{m}$ silver-coated, hollow glass spheres at a density of $0.1 \mathrm{ppp}$. These particles, when illuminated by laser light, allow for cameras to identify the movement of fluid around the plate, provided the particles follow the flow faithfully.

The behaviour of particles suspended in fluid flow is characterized by a dimensionless number, known as the Stokes number. The Stokes number is defined as the 
ratio of the characteristic time of a particle to a characteristic time of the flow, and represents the fractional reaction time of a finite-mass particle to changes in the surrounding fluid. A particle with a low Stokes number tends to follow fluid streamlines, while a particle with a large Stokes number is dominated by inertia and tends to continue along its initial trajectory, ignoring local fluid velocity. The Stokes number is calculated using:

$$
\mathrm{Stk}=\frac{\tau u_{\infty}}{d_{c}},
$$

where $\tau$ is the relaxation time of the particle (the time constant in the exponential decay of the particle velocity due to drag), $u_{\infty}$ is the velocity far from the body in the flow, and $d_{c}$ is the characteristic length scale of the body. The time constant $\tau$ associated with low Reynolds number flows can be calculated as:

$$
\tau=\frac{\rho_{d} d_{d}^{2}}{18 \mu},
$$

where $\rho_{d}$ is the density of the particle, $d_{d}$ is the diameter of the particle, and $\mu$ is the dynamic viscosity of the surrounding fluid. Since the maximum Reynolds number of the seeding particles was $\mathrm{Re}=100$, Equation 4.4 is a reasonable approximation. The Stokes number for particles in the flow can then be estimated using:

$$
\mathrm{Stk} \approx \frac{\rho_{d} d_{d}^{2} u_{\infty}}{18 d_{c} \mu}
$$

Substituting the diameter of the plate in for the characteristic length scale, and using $U_{f}=0.39 \mathrm{~m} / \mathrm{s}$ (equivalent to $\mathrm{Re}=125,000$ ) as the final towing velocity, the Stokes number for particles in the flow is approximately $\mathrm{Stk} \approx 10^{-3}$. Since Stk $<0.1$, it can be assumed that tracing errors are less than 1\%, as shown by Tropea et al. (2007). 
A right-hand coordinate system was adopted such that the $x-, y-$, and $z$-axes were oriented in the streamwise, wall-normal, and spanwise directions, respectively. The flow field was captured using a Photron SA4 high-speed camera, which has a resolution of $1024 \times 1024 \mathrm{px}$, and was operated at a frame rate of $1000 \mathrm{~Hz}$. A 40 mJ per pulse Photonics Nd:YLF high-speed laser was used to create a laser sheet approximately $2 \mathrm{~mm}$ thick in the $x y$-plane. The FoV used for all measurements was approximately $1.2 D \times 1.2 D$ in size and was situated at the horizontal midspan of the plate along the lower half of the sting, as shown in Figure 4.3.

With the original intention of measuring the development of a single reference plane in the streamwise direction, six sets of PIV measurements were taken in consecutive downstream fields of view, shown in Figure 4.4. Instead of moving the camera and laser system for each successive FoV, the initial $x$-position of the plate was varied. Each FoV had a 10\% overlap with each of its adjacent neighbours in the streamwise direction. The towing motion was repeated and recorded for $N=10$ identical runs for each FoV to phase-average velocity field results, a technique that reduces the error by a factor of $\sqrt{N}$ compared to the error associated with a single run. A multi-frame, single-exposure PIV algorithm in DaVis 8, a software package by LaVision GmbH, was used to cross-correlate sequential images pairs, generating displacement vectors, as described by Raffel et al. (2007). This software calculated particle positions to within sub-pixel accuracy, at approximately $\pm 0.1 \mathrm{px}$. With an average pixel displacement between frames of $5 \mathrm{px}$, the resulting error in particle displacement is roughly $2 \%$, corresponding to a maximum uncertainty of $4 \%$ for normalized velocity and $8 \%$ for normalized vorticity.

Velocity vectors were calculated at a final correlation window size of $32 \times 32 \mathrm{px}^{2}$ 


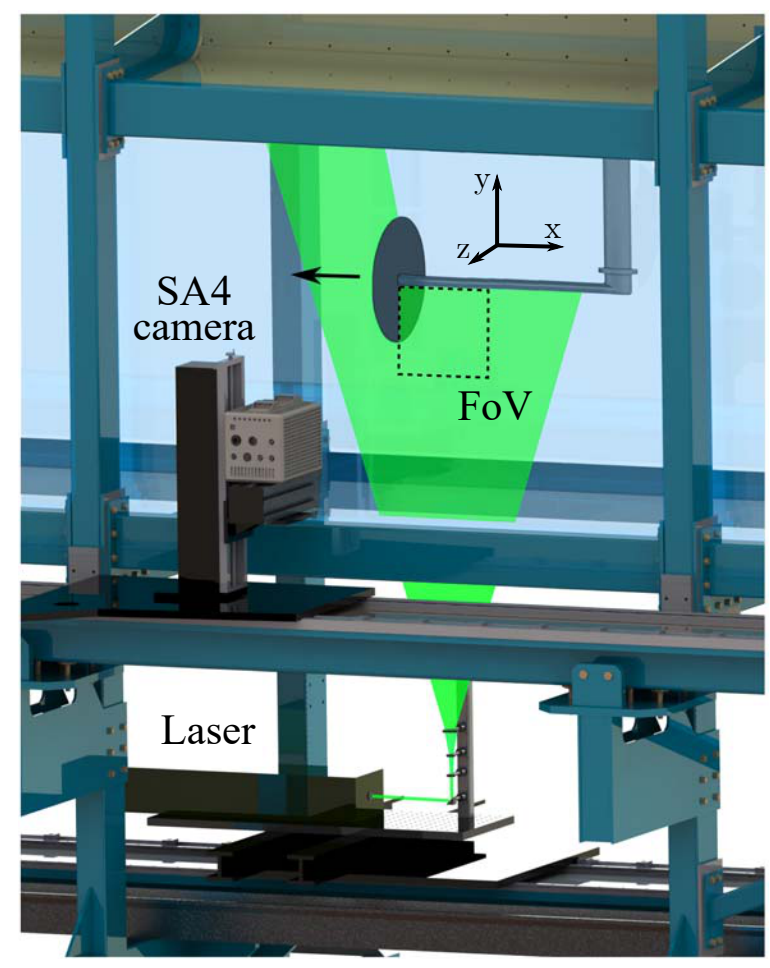

Figure 4.3: The experimental set-up for PIV measurements, including the location of the high-speed laser and camera, and the approximate size and orientation of the field of view (FoV).

with $75 \%$ overlap. The final velocity data from each phase-averaged FoV was stitched together to form a final dataset of length $7 D$ in the streamwise direction, with the plate initially positioned $0.5 D$ from the $+x$ boundary of the first FoV.

In order to produce Lagrangian measurements from a nominally Eulerian flow field, 30,000 synthetic particles were seeded randomly into the two-dimensional velocity field at $t^{*}=0$. This seeding density corresponds to a spatial particle density of $5 \times 10^{-3}$ ppp, which is just below the upper particle density limit achievable by stereoand tomographic-PIV techniques of $10^{-2} \mathrm{ppp}$, as described by Raffel et al. (2007), and significantly below the limit of 0.125 ppp of the Shake-The-Box algorithm, as described by Schanz et al. (2016). Each particle was propagated in the direction of the 


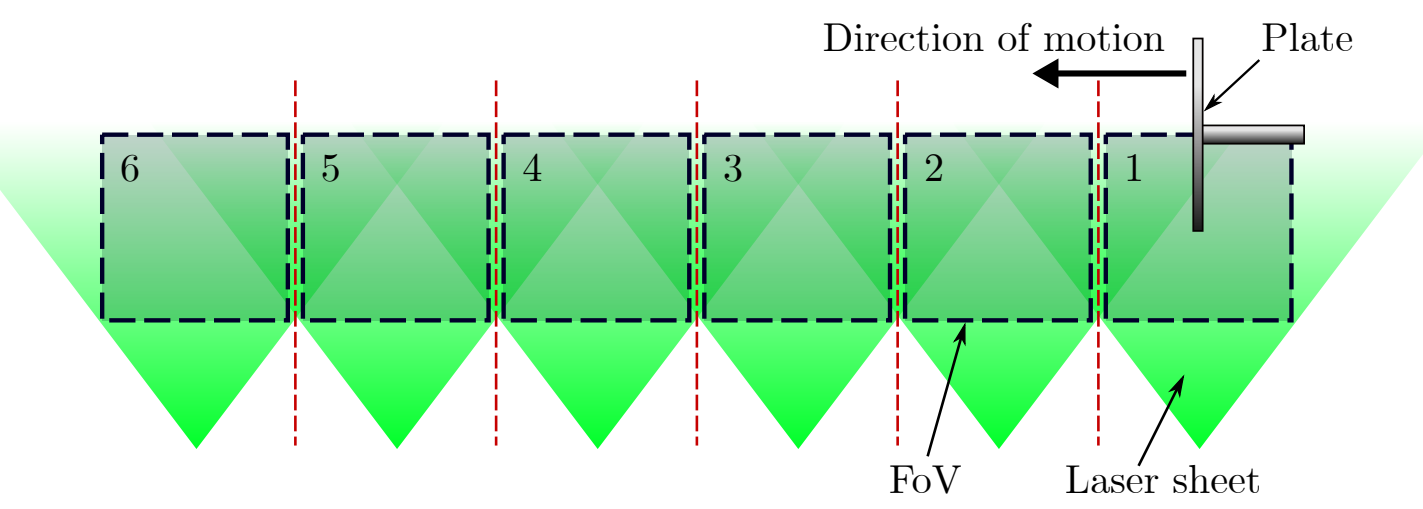

Figure 4.4: Multiple fields of view for PIV measurements were arranged in the streamwise direction. In reality, the laser and the camera remained stationary and the plate's starting position was altered to change the effective FoV captured. Red dashed lines represent locations where there exists a $10 \%$ overlap between FoVs, however neighbouring FoVs have been separated for illustrative clarity.

local velocity for a time duration $\Delta t$ equal to the inverse of the repetition rate of the laser and camera system, $\Delta t=1 /(1000 \mathrm{~Hz})=10^{-3} \mathrm{~s}$. At each successive time-step, the propagation procedure was repeated with the advantage that the final pathline data was extended far downstream so as to capture the full drift volume developed from a single reference plane, rather than be limited to a single FoV. This particle pathline data is visualized in Figure 3.3 and is used for drift volume calculation.

The assumption of axisymmetry, justified by the results of Fernando and Rival (2016), about the flow around the plate's circumference was used to convert the nominally two-dimensional drift area into a full three-dimensional drift volume by means of cylindrical integration. At each timestep, individual fluid particles were revolved around the plate's axis of symmetry for the calculation of a three-dimensional drift volume. An example of fluid particles displaced from a single reference plane is illustrated in Figure 4.5. Particle trajectories and their integration paths are shown relative to the plate's axis of symmetry. 


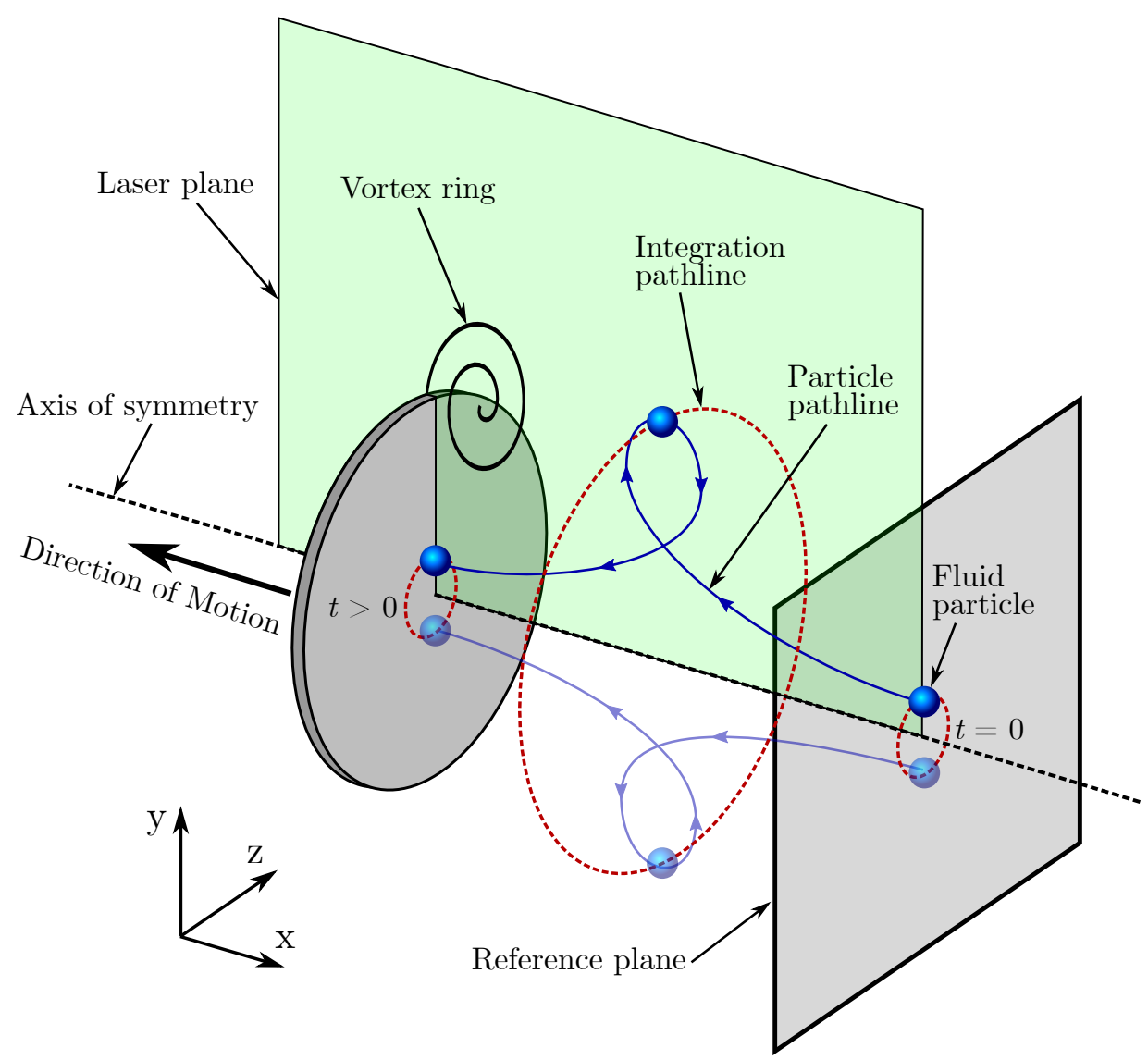

Figure 4.5: Cylindrical integration around the plate's axis of symmetry is calculated for individual fluid particles. Particle pathlines (blue lines) are shown for particles (blue spheres) originating from a single reference plane. Integration pathlines (red dashed lines) are shown for a fluid particle at three distinct timesteps. The laser sheet (green) is shown in the $x y$-plane. 


\section{Chapter 5}

\section{Results and Discussion}

The following chapter discusses the results obtained from the experiment performed on an accelerating circular flat plate. Optimal parameters for the multi-plane driftvolume technique are discussed and the limitations are described. The force analysis is decomposed into two time intervals, between which the experimental kinematics change, to show the strengths and limitations of the proposed Lagrangian drift-volume force estimation technique compared to contemporary Eulerian wake-vortex methods.

\subsection{Multi-Plane Drift Volume}

Positive (downstream) and negative (upstream) drift volumes were calculated at each time step using the aforementioned multi-plane drift-volume calculation method. The number of reference planes in the multi-plane drift-volume force estimation technique was at first varied from $N=5$ to $N=80$, where the distance between consecutive planes was halved at each iteration. Thus, the values of $N$ analyzed were $N=$ 5, 10, 20, 40 and 80. An illustration of $N=20, N=40$ and $N=80$ is seen along the top row of Figure 5.1, where only the streamwise range of $-1.5<x / D<0.5$ out of the total range of $-6.5<x / D<0.5$ is shown. Subsequent rows in Figure 5.1 show 

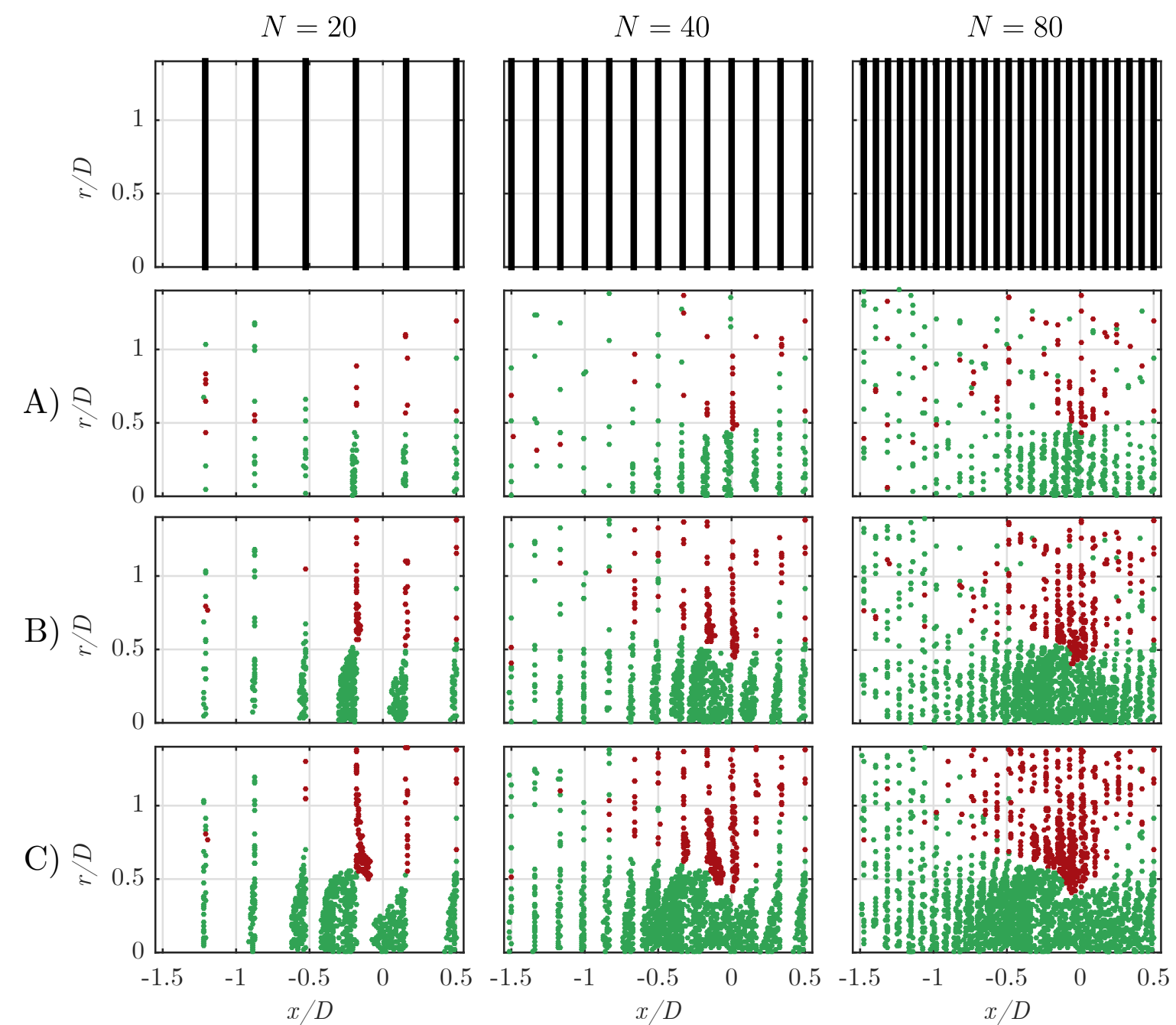

Figure 5.1: Positive drift (green) and negative drift (red) are shown for a varying number of reference planes $N$ at various times $t^{*}$. Shown here are illustrations for $N=20$ (left), $N=40$ (middle), and $N=80$ (right). Multi-plane drift is shown for $t^{*}=0.13(A), t^{*}=0.25(B)$, and $t^{*}=0.38$ $(C)$. The plate, initially at $x / D=0$ with its axis of symmetry located on the $r / D=0$ plane, accelerates from rest at in the $-x$ direction and over the time interval $0<t^{*}<0.25$.

the developed multi-plane drift, at times $t^{*}=0.13, t^{*}=0.25$, and $t^{*}=0.38$.

The main advantage of having $N$ as large as possible is that the drift-volume is developed less intermittently in space, seen in the discrepancy between the columns 
of Figure 5.1. At $N=80$, the effects of discrete reference planes are minimized by allowing the development of a single quasi-continuous drift-volume. Lower values of $N$ have qualitatively identifiable regions of drift (green/red) and no-drift (white) between reference planes, which hinders the incorporation of all moving fluid in the total force calculation. For $N \gg 80$, exemplified by the $N=100$ curve in Figure 5.2, fluctuating errors in the velocity field calculated from PIV give artificial streamwise movement of fluid particles and incorrectly estimate the total drift volume.

PIV error quantification is investigated rigorously by Sciacchitano et al. (2013), Wieneke (2015), and Sciacchitano and Wieneke (2016), who show the strengths and weaknesses of various experimental setups for PIV. In the current test case, the physical calibration target, used to convert camera pixels into physical locations in space, is considerably smaller than the FoV. Because the calibration target is located in the center of the FoV, PIV errors associated with calibration give sporadic results near the outer edges of the FoV, whereas more accurate results are obtained in the center region. When $N$ is increased past approximately $N=90$, these spurious vectors near the edge of the FoV cause particle displacements to become erroneous. Errors are also induced where velocities are very small, typically far from the body, since seeding particle displacements between sequential camera frames can be too small for PIV correlation peaks to correctly define positive or negative $x$-direction displacements. An incorrect determination of small $x$-displacements will, due to the small gap between reference planes associated with large $N$, produce erroneous drift volume contributions.

Figure 5.2 shows only $N \leq 100$ since these values of $N$ are relevant to the discussion of multi-plane versus single-plane drift-volume methods. $N>100$ is not plotted 
because above $N \approx 90$ the current dataset generates false drift-volume regions near the FoV extremities where artificial drift volume contributions are on the same order of magnitude as near-body regions of drift, a non-physical artifact. In the current test case, a window of approximately $70<N<90$ exists where the drift-volume force estimation technique produces results that agree well with the measured force. The value of $N$ that produces reliable results is on the order of one reference plane per streamwise correlation window. In the current test case, $32 \times 32 \mathrm{px}^{2}$ correlation windows were used over a sensor size of $1024 \times 1024 \mathrm{px}^{2}$ with approximately 25 reference planes per FoV (corresponding to $N=80$ ). More accurate PIV data in the far field, from better calibration, will allow the optimal range of $N$ values to extend higher without sacrificing accuracy in the method.

The rows of Figure 5.1 show the drift-volume development over time. Green and red particles (positive and negative drift) become more dense near the plate's center at $r / D=0$ as $t^{*}$ increases. When $t^{*} \geq 0.5$, clipping occurs at the edge of the FoV since non-negligible far-field fluid $x$-direction displacements tend toward larger $r / D$ with increasing $t^{*}$. Therefore the confidence interval for the drift-volume calculation in this experiment is limited to $0 \leq t^{*}<0.5$.

\section{$5.2 \quad$ Force Analysis}

Using Darwin's drift-volume, added-mass equivalence (Equation 2.21), the net downstream drift calculated from the multi-plane approach is, at each time step, made equal to the instantaneous added mass of the flat plate. Subsequently, the total force acting on the plate is calculated using Equation 2.8, where the control surface terms of Equation 2.4 assumed to be negligible, and is shown in Figure 5.2 for each value 
of $N$ considered.

The current drift-volume force estimation technique is compared to the measured force over the time interval $0 \leq t^{*} \leq 1$ and is shown in Figure 5.2. The estimated forces converge to the measured force for values of $N$ between $N=70$ and $N=90$, during the acceleration phase of $0<t^{*}<0.25$. Below $N=70$, the calculated force is not representative of the measured force since some remaining errors associated with single-plane drift-volume calculations still exist. These calculation artifacts are explained in detail in $§ 5.2 .4$. Similarly, above $N=100$, the calculated force begins to diverge from the measured force in the region of peak force due to finite errors in particle positions, stemming from small inaccuracies in the velocity field, as explained in the previous section.

Also compared in Figure 5.2 is the added-mass force calculated from potential flow theory, shown in blue. This force curve is calculated using Equation 2.11, where the added-mass coefficient for a circular flat plate is $c_{\mathrm{ii}}=8 / 3$ (from Brennan (1982)). The added-mass force in potential flows exists purely within the acceleration phase of the motion, over the time interval $0<t^{*}<0.25$, and is proportional to the acceleration of the body. Figure 5.2 clearly shows that the classical added-mass force is insufficient for predicting the total force on an accelerating plate; it is unable to capture either the increasing force development or the peak force on the plate.

For sufficiently large $N$, the Lagrangian drift-volume force estimation method produces results that initially track the measured force well. The force estimate for $N=80$ is compared to the measured force in Figure 5.3. In the region of $0<t^{*}<$ 0.25 , the average difference between measured drag coefficient $C_{D_{M}}$ and drift-volume 


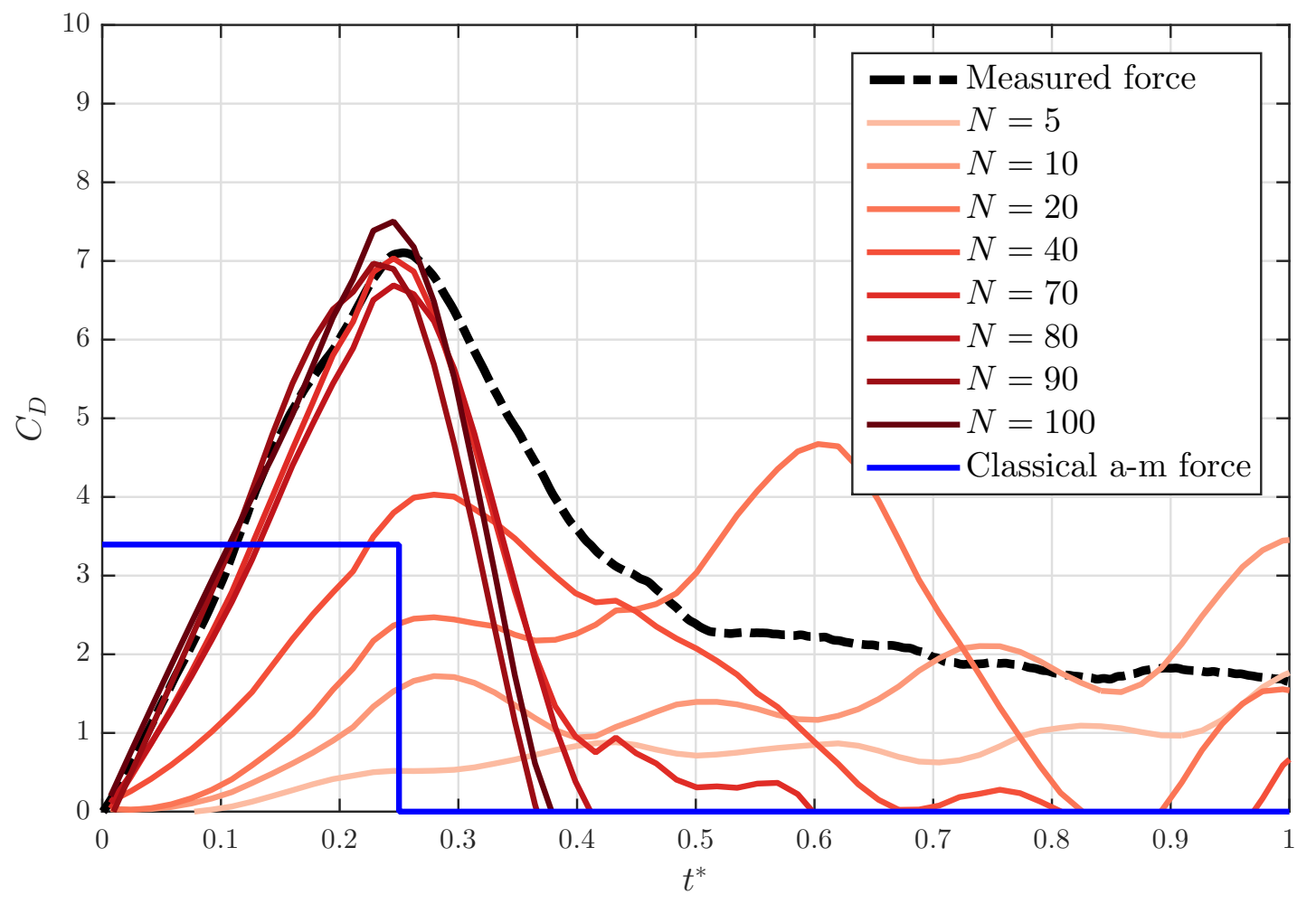

Figure 5.2: Forces are calculated from the multi-plane drift-volume approach for a varying number of reference planes $N$. The measured force from a physical force transducer (black dashed line) is shown for comparison. The classical added-mass force from potential flow (blue) is directly proportional to the acceleration of the body; however, it does not match up with the measured force peak. The acceleration occurs over the time interval $0<t^{*}<0.25$.

drag coefficient $C_{D_{D V}}$ is

$$
\left|C_{D_{M}}-C_{D_{D V}}\right|_{\text {avg }}=0.24 .
$$

When this mean difference is compared to the peak measured drag coefficient of $\left(C_{D_{M}}\right)_{\max }=7.1$, the result is an average of $3 \%$ error. 


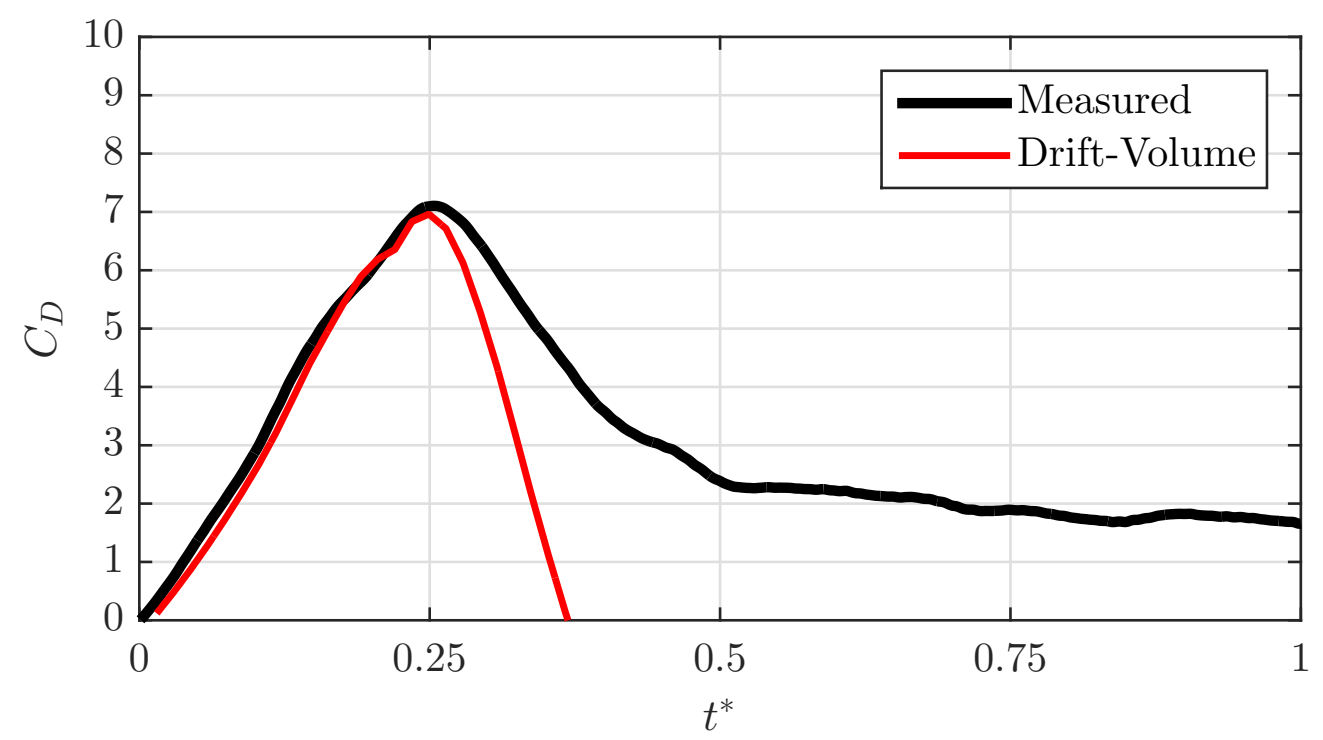

Figure 5.3: Measured force (black) is compared to the multi-plane drift-volume technique $(r e d)$. During the acceleration period, $0 \leq t^{*} \leq 0.25$, the driftvolume method follows the measured force curve with good agreement. After $t^{*}=0.25$, the discrepancy between the two curves increases beyond the limits of experimental error. The measured force curve is not well tracked in its relaxation toward state-state conditions.

\subsubsection{Transition Toward Steady State}

In Chapter 2, while deriving the added-mass force in Equation 2.7, the assumptions made when moving from Equation 2.6 to 2.7 explain the lack of collapse of the driftvolume force estimation curve onto the measured force curve past $t^{*}=0.25$. The drift-volume method calculates a force that drops off sharply after the end of the acceleration phase. This drop-off is consistent with the theory in Chapter 2 that the added-mass force, and hence the current force estimation method that neglects the control surface terms in Equation 2.4, is not intended to calculate the drag on a body with zero acceleration. 
When the plate travels at constant velocity, herein after referred to as the "steadystate" region, the time rate of change term in Equation 2.4 tends to zero due to the decreasing time dependence in the flow field. The convective, pressure and viscous terms however become non-negligible in their contribution to the force history as the plate undergoes constant velocity motion. More importantly, the main assumption made in Chapter 2 that allows the use of Equation 2.8 in the drift-volume method is one that breaks down after the body is no longer accelerating. Equation 2.9 shows that the only fluid considered in the time-dependent term of Equation 2.4 is the fluid that moves in phase with the body, the added mass, which is said to no longer be accelerating when the body is no longer accelerating. This assumption is consistent with the results, implying that the drift-volume method, using only the unsteady term to estimate the total force, does not have the ability to estimate an accurate drag force past $t^{*}=0.25$.

The measured force curve in Figure 5.3 suggests that, even though the body has stopped accelerating at $t^{*}=0.25$, the surrounding fluid has a non-zero relaxation time as the measured force is reduced from its peak over the interval $0.25<t^{*}<1$. From $t^{*} \approx 0.5$ onward, the measured force curve tends toward steady-state and can be explained by the vortex dynamics explored by Fernando and Rival (2016).

\subsubsection{Comparison to Modern Eulerian Methods}

The inherent advantage of the current force estimation technique presented is that, unlike modern Eulerian techniques, many of the error producing operations are not required to be performed. These noise amplifying processes are avoided since the drift volume is calculated only from particle displacements in the flow field, reducing the 
importance of having high quality data near the wall and in regions of high shear.

Near-wall errors in optical measurements can stem from body reflections, blockages in camera visibility, and PIV biasing. The light source, often a high-powered laser, can cause bright reflections on the body's surface that interfere with accurate particle identification near the fluid-surface interface. The movement of the body, combined with camera parallax limitations, can prevent important near-wall regions from being visible, especially in multi-camera setups for three-dimensional imaging. Even with perfect near-wall visibility, biases associated with PIV are demonstrated by Kähler et al. (2012) to have negative effects on near-wall accuracy.

These errors are costly in techniques that rely on near-wall spatial gradients but are less relevant in the current method by making use of the continuous nature of the drift volume and the fact that the drift volume calculation is an integral measurement. For example, a positive drift volume is a region of fluid contained by reference planes that have deformed around a translating body, illustrated in Figures 2.4 and 3.5. The drift volume is continuous in space, containing no discontinuities or holes, and is contained by the deformed reference planes. If, for instance, poor-quality flow-field data in the near-wall region has yielded an unexpected gap in particles contributing to the positive drift volume (manifested either as stationary particles or reverse-flow particles where positively contributing particles are predicted), the gap region can be assumed to also contribute to the positive drift volume. This assumption can be made near the body since fluid on and near the body is moving at or near the velocity of the body. Secondly, the drift volume is calculated in a way where each fluid particle contributing to the total drift volume is equal to each and every other particle. Fluid particles in regions containing the highest accelerations, gradients and 
hence the highest errors, are not as important in the drift-volume method as they are in the Eulerian approaches. The main advantage of the drift-volume approach is that all fluid particles are equally-weighted in their contribution to the drift volume, where the large majority of these particles are located away from the inaccurate near-wall regions.

To show a comparison between the existing Eulerian methods and the new Lagrangian method, a new row is added to Table 2.1 for the multi-plane drift-volume technique, now shown in Table 5.1. While only the unsteady term of the drift-volume method has been considered in the current analysis, the method still has control surface integrals identical to those in the DMT and classical methods that contribute to extra derivative terms. To give a fair comparison to the other methods presented, three spatial derivatives and one temporal derivative are added to the drift-volume method in Table 5.1 since they are required to compute the convective and viscous control surface integrals as well as Poisson's equation for pressure.

A major improvement of the drift-volume method over the existing methods is that the temporal derivative required is the time rate of change of a spatial integration, that is, a temporal derivative of the drift volume as a whole, rather than a derivative at each point in space. This type of derivative is considerably less sensitive to noise than the point-wise derivatives conducted in the Eulerian techniques (Burden et al. (2015)). This main benefit can be explored further if the terms of Equation 2.8 are inspected more closely. The added mass is calculated from the drift volume, which is an integral measurement calculated from the accounting of fluid particles. The time rate of change of the drift volume, as mentioned, is the derivative of an integral quantity, not requiring individual derivatives at each point in space. The body 
Table 5.1: The accuracy of the Lagrangian drift-volume force estimation technique is compared to the aforementioned Eulerian force estimation techniques. Each multiplication, temporal derivative and spatial derivative propagates errors throughout the calculation. The drift-volume technique has the lowest value in three of the four columns of error propagating calculations.

\begin{tabular}{lllll}
\hline Method & No. terms & $\begin{array}{l}\text { No. mul- } \\
\text { tiplica- } \\
\text { tions }\end{array}$ & $\begin{array}{l}\text { No. tem- } \\
\text { poral } \\
\text { deriva- } \\
\text { tives }\end{array}$ & $\begin{array}{l}\text { No. } \\
\text { spatial } \\
\text { deriva- } \\
\text { tives }\end{array}$ \\
\hline \hline $\begin{array}{l}\text { Classical formulation } \\
\begin{array}{l}\text { Derivative-moment } \\
\text { transformation method }\end{array}\end{array}$ & 4 & 2 & 2 & 3 \\
$\begin{array}{l}\text { Vorticity formulation } \\
\text { Drift-volume method }\end{array}$ & 9 & 3 & 2 & 3 \\
\hline
\end{tabular}

velocity and body acceleration are well-defined functions prescribed by the kinematics of the motion; they are also not calculated at each point in space. As a combined result, the drift-volume method removes the major sources of error associated with the unsteady terms seen in modern Eulerian techniques.

Going into further detail, the drift-volume method has specific advantages over each of the compared force estimation methods:

- Classical Formulation: PIV errors in near-wall and high-shear regions produce inaccurate fluid accelerations where force estimate calculations are most critical. Calculation of the drift volume does not rely on fluid acceleration;

- Vorticity Formulation (Noca et al. (1997)): Although there is a no pressure term in this formulation, the effect of compounding errors from numerous multiplications and derivatives significantly hampers the ability to estimate accurate forces from sub-optimal data. In a similar manner, the wake-vortex driftvolume approach of Dabiri (2005) also uses the hydrodynamic impulse term 
of the vorticity formulation, giving it similar error propagation characteristics. The drift-volume is much simpler mathematically, and propagates less error comparatively;

- Derivative-moment transformation: The DMT method and the drift-volume method require identical convective, pressure and viscous control surface terms; however, the unsteady term in the DMT method is susceptible to errors from velocity gradients passing through the control surface, as described by Mohebbian and Rival (2012). The unsteady term in the DMT method requires a moment arm calculation of the surface-normal velocity, a noise amplification problem. Conversely, the drift-volume method has the ability to produce less error when regions of high shear pass through the control surface boundaries since the only calculation requirement is that particles are tagged either as positive or negative contributors to the drift volume, regardless of the existence of any local spatial gradients.

\subsubsection{Direct Comparison to the Wake-Vortex Method}

A comparison is made between the novel Lagrangian drift-volume force estimation technique and a leading Eulerian wake-vortex method, described by Wu et al. (2006) and introduced in Section 2.4, using the same PIV dataset. The comparison of the two force estimation methods is shown in Figure 5.4. The force estimate derived from the wake-vortex method (Equation 2.14) gives a poor force estimate over the time interval $0<t^{*}<0.5$ because the vorticity is high and localized near the wall, producing large errors; refer to Kähler et al. (2012) for details regarding near wall uncertainty in PIV. The resolution required to obtain sufficient near-wall data for 


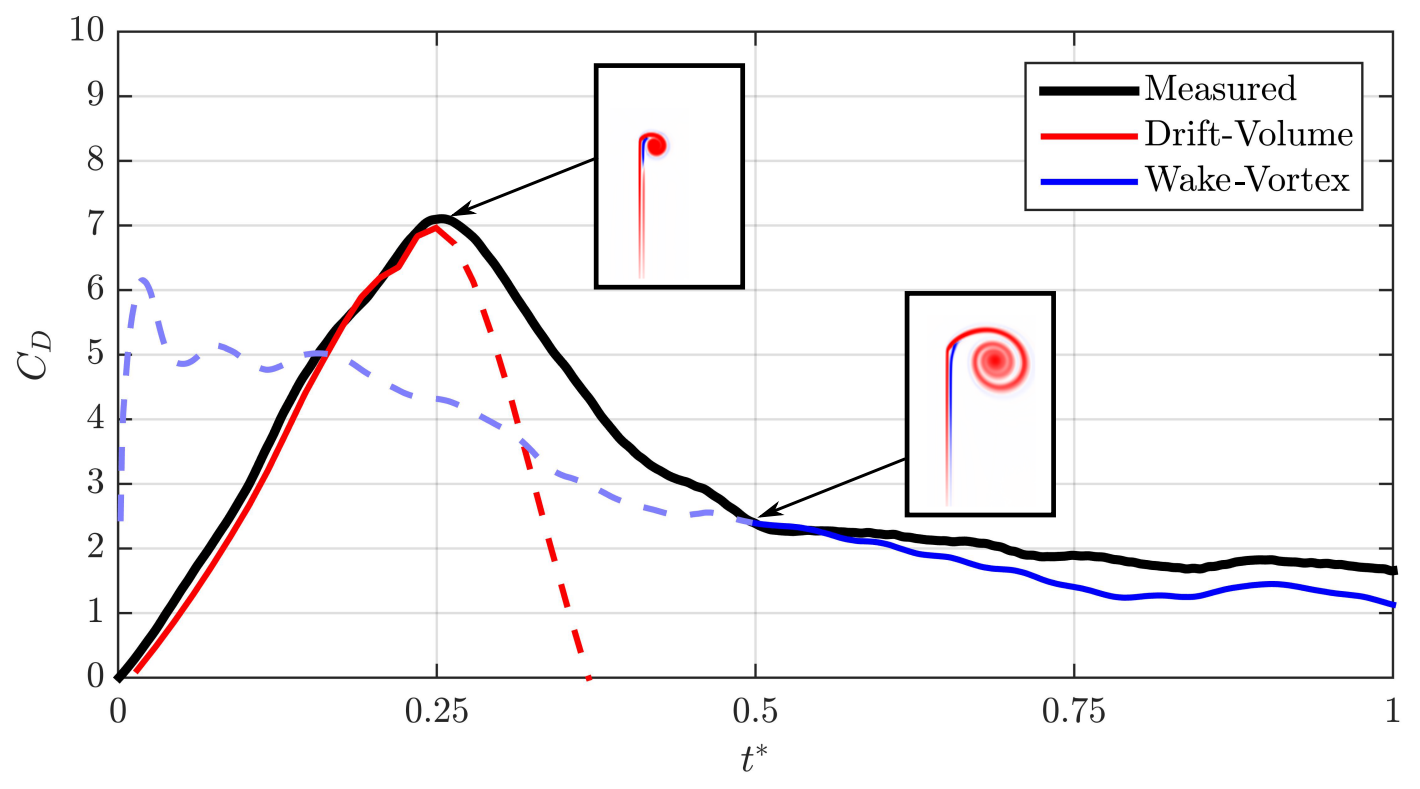

Figure 5.4: Measured force (black line) is compared to two different methods of estimated force: the multi-plane drift-volume technique (red) and a wakevortex formulation from $\mathrm{Wu}$ et al. (2006) (blue). The dashed colored lines represent the region for each technique where the force estimate is no longer accurate, based on known limitations of the measurement techniques. The state of the wake is shown for two times, $t^{*}=0.25$ and $t^{*}=0.5$, where the colours, blue and red, in the inset images represent positive and negative $z$-direction vorticity $\omega_{z}$, respectively.

accurate force estimation is such that the FoV would no longer be large enough to capture all forces associated with wake vortices due to the limited dynamic spatial range of modern experimental hardware for PIV, as addressed by Adrian (1997). Once the starting vortex has grown to near its full size, after $t^{*} \approx 0.5$, the Eulerian wake-vortex measurements align well with the measured force since most of the force contributing vorticity is now in the wake and far from the body's surface.

Theoretically, if the entire flow field was sampled with sufficient resolution to 
capture even the smallest regions of vorticity-containing fluid, the wake-vortex method from Wu et al. (2006) would produce full and exact time-resolved forces acting on the plate. Since the majority of the energy-containing fluid for $t^{*}>0.5$, contributing to drag, is located in the wake (away from the wall) rather than in the boundary layer (near the wall), the vorticity field has lower error and Equation 2.14 can be used with good reliability. Over the time interval where the energy-containing fluid is wound up tightly within the boundary layer, the current drift-volume method instead uses the far-field information to estimate the net force on the body, avoiding the rampant error production associated with calculating vorticity near the wall.

The region of confidence for the drift-volume method (where CS terms are neglected) extends from $t^{*}=0$ to $t^{*}=0.25$, whereas the region of confidence for the wake-vortex method is approximately $t^{*}>0.5$. The measured force past $t^{*}=0.5$ approaches a steady-state value, explained by the vortex ring dynamics described by Fernando and Rival (2016) and can be well calculated using conventional Eulerian force estimation techniques. The period of most interest, however, is the aggressive acceleration and the force peak, as these regions dictate the structural loading requirements and maximum force observed on bodies submersed in fluids, both of which are accurately captured by the drift-volume technique.

\subsubsection{Force Estimation Artifacts}

As referred to earlier, there are drift-volume calculation artifacts that explain why lower values of $N$ do not produce accurate results in the multi-plane drift-volume force estimation technqiue.

Firstly, some fluid particles needed to calculate the drift volume can be beyond 


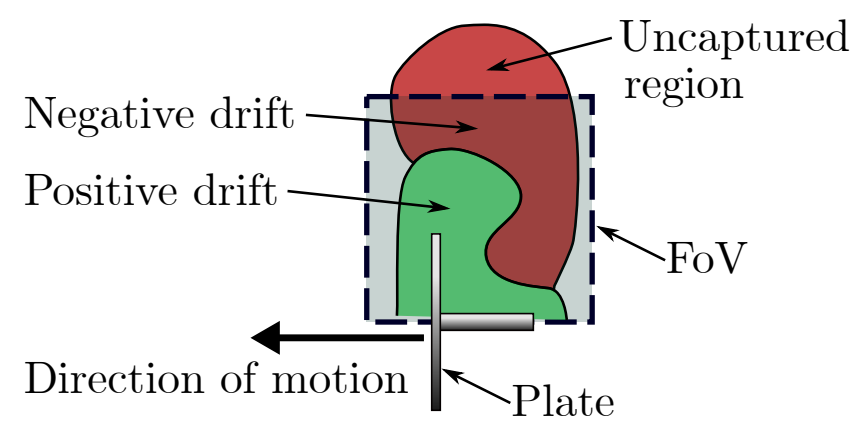

Figure 5.5: Error is induced in the drift-volume calculation when fluid particles displaced in the streamwise direction are outside of the field of view. In this illustration, the negative drift volume contribution is underestimated.

the limits of the FoV. As the starting vortex grows in time, a larger region of fluid is disturbed and fluid particles farther away from the body, ultimately outside the FoV, have non-negligible streamwise displacements. These uncounted fluid particles can reduce either the amount of positive or negative drift, producing artificial bias. This type of missing information is illustrated in Figure 5.5.

Next, the rate of change of the net drift-volume is influenced by the spacing of the $N$ reference planes. Artificial peaks can arise later in time from tagged fluid that has not yet passed through a reference plane, due to large inter-plane spacing, but then later passes through a downstream reference plane. Depending on the position and spacing of the reference planes, the fluid trapped by the start-up vortex may only be incorporated into the drift volume later in time when the vortex passes through a reference plane that is farther downstream, shown in Figure 5.6. The instantaneous rate of drift-volume development is increased when the starting vortex ring passes through the next reference plane. This increase leads to a spike in the $d m_{a} / d t$ term in Equation 2.8, producing the artificial force peak, seen most prominently in the second $N=20$ peak in Figure 5.2. 


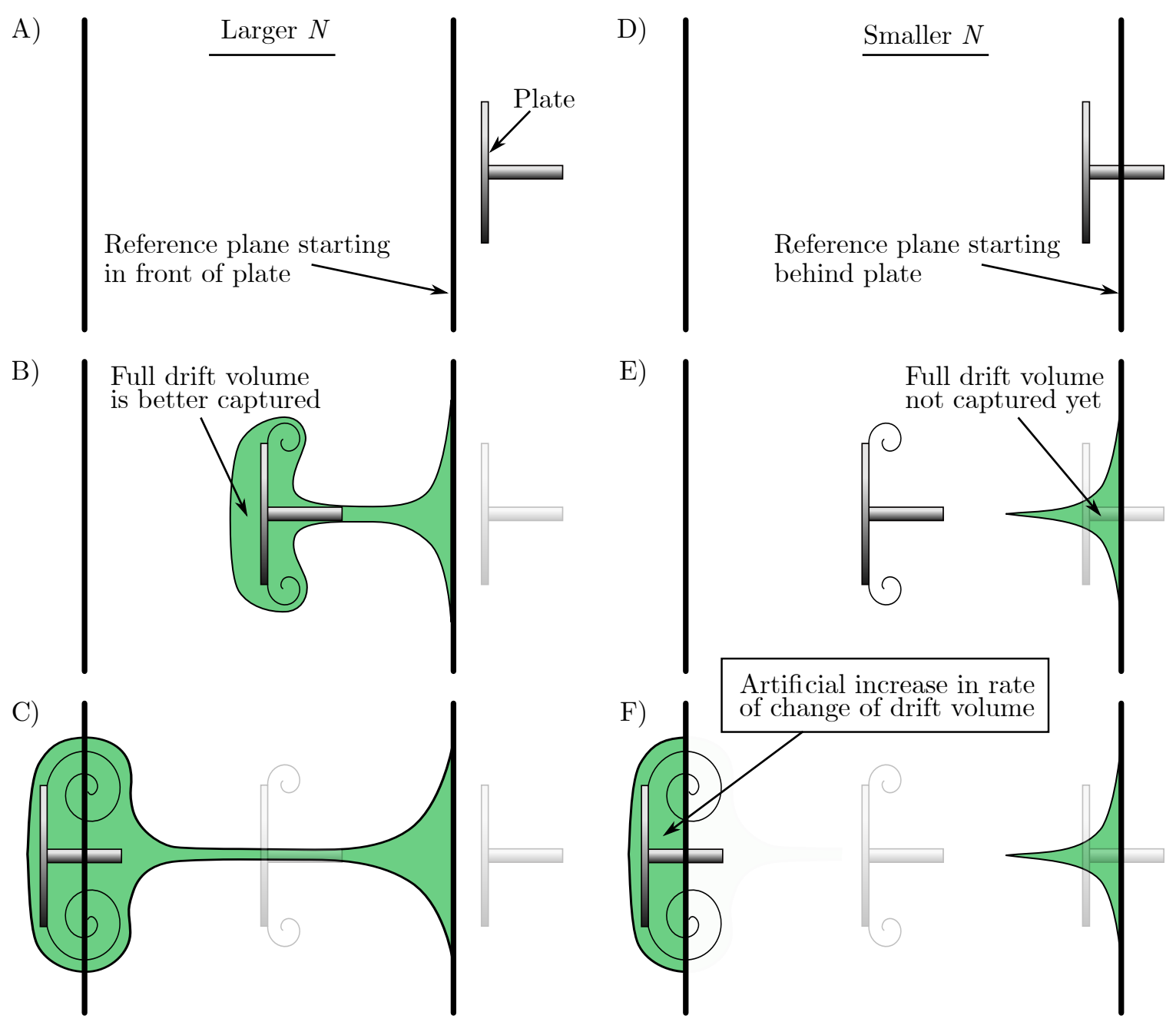

Figure 5.6: Two values of $N$ are compared to show the artificial force increase associated with lower $N$ for the case where displaced fluid in the start up vortex is not captured until later in time. Closer reference plane spacing, higher $N(\mathrm{~A}, \mathrm{~B}, \mathrm{C})$, is compared to larger reference plane spacing, lower $N$ (D,E,F). When the plate accelerates from rest, as shown in the progression from top row to bottom row, smaller values of $N$ do not capture the downstream fluid displacement contained within the starting vortex (E versus B). Later in time, the vortex passes through the next downstream reference plane, and a rapid increase in the measured drift volume occurs $(\mathrm{F})$. This increase results in an artificial peak in the $\partial m_{a} / \partial t$ term of Equation 2.8, whereas this peak does not occur for large $N$ since this drift was previously captured (C). 


\section{Chapter 6}

\section{Conclusions}

The proposed unsteady Lagrangian force estimation technique and experimental investigations of the thesis are summarized, and the major results are reviewed. Next, the main conclusions for future experimental implementation are stated. Finally, recommendations are made for future work in this area.

\subsection{Conclusions}

A new method for non-intrusively estimating the unsteady force on an accelerating body has been developed. This method uses the proposition that instantaneous added mass can be derived from the development of multiple Lagrangian drift volumes, using the drift-volume, added-mass equivalence proposed by Darwin (1953). It was shown that the number of reference planes $N$ in the multi-plane drift-volume calculation is critical to alleviating the residual inaccuracies of single-plane drift-volume calculations while also avoiding artifacts stemming from PIV uncertainty. Using a value of $N$ within the optimal range, the unsteady forces calculated from the drift-volume method were shown to align well with the measured force on a circular flat plate during 
acceleration from rest. Once the plate was no longer accelerating, and constant velocity was reached, mathematical assumptions associated with the drift-volume force estimation method no longer held. These assumptions include the attribution of the entire flow field's acceleration to a single fluid mass and the neglect of control surface integrals, which become important at steady-state. Because the fluid associated with the added mass of a body moves in phase with that body, the added-mass force disappears when the acceleration is equal to zero, even though the measured force does not immediately drop. The critical aspects of understanding unsteady flows, relating to peak and time-resolved body loading, often lie within the acceleration phase of the kinematics, which the proposed method captures to within $3 \%$ error in the current experiment.

The results discussed in Chapter 5 indicate that the drift-volume method has the ability to capture the full reaction force of an accelerating motion and has many advantages over current Eulerian techniques. Near-wall biases, compounding uncertainty propagation and regions of high shear all contribute to a reduction of accuracy in the Eulerian techniques; however, the Lagrangian drift-volume method manages to avoid these sources of error through the calculation of the drift volume. As opposed to needing high quality data everywhere in space (classical and vorticity formulations) or on a control surface (DMT method), the drift-volume method instead uses an integral relationship between particle displacements and the unsteady force. Calculations based on this integral measurement are less prone to propagating errors and are ultimately advantageous in the estimation of unsteady forces. 
The drift-volume force estimation technique was compared to a modern vorticitybased Eulerian force estimation technique, the wake-vortex force formulation presented by Wu et al. (2006). During the acceleratory phase, where the vorticity is centralized near the body's surface, the wake-vortex method was unable to capture the added-mass force peak seen in the measured force curve. Once the majority of the vorticity containing fluid was convected into the wake, the vortex-force method became well-aligned with the measured force since near-wall PIV errors were no longer present. While the confidence interval for the wake-vortex method is limited by the dynamic spatial range of current PIV technology, the drift-volume method does not share the same fate due to the absence of spatial gradients in its formulation. The unsteadiness of the motion is well-captured by the drift-volume method because of the derivation from added-mass concepts, but without the control surface terms of Equation 2.4 the method breaks down in the transition toward steady-state conditions.

This unique force estimation method opens the door for unsteady force analysis in experiments where a physical force sensor is not feasible and where spatial resolution is not sufficient for force to be accurately estimated using the currently available Eulerian approaches. Further validation on highly-separated, highly-unsteady flows is yet required to prove the full benefit of this drift-volume force estimation technique; however, this first investigation serves as the proving ground for a new trend toward the use of Lagrangian measurement techniques for fluid dynamics analysis. 


\subsection{Future Experimental Considerations}

If possible, an LPT approach, such as Shake-The-Box, should be used due to its superior algorithm for particle pathline reconstruction. As shown by Kähler et al. (2012), particle tracking methods do not suffer the same near-wall bias errors as PIV measurements and have a significantly higher dynamic spatial range. Even though the force output in the drift-volume method is less sensitive to velocities near the wall, as those particles represent only a fraction of the total drift volume, still higher accuracy measurements are possible with more advanced particle tracking algorithms. One caveat is that it may not be possible to implement three-dimensional LPT since parallax effects and visual blockages from a solid body may hinder the acquisition of data near the body, in which case an alternative three-dimensional approach may be necessary.

In a similar manner to how Green et al. (2011) measured the wake on a pitching panel, a stack of two-dimensional PIV planes can be used to produce threedimensional gridded flow data. Even without calculating the out-of-plane component of velocity (requiring a multi-camera setup, encountering the same parallax issues as LPT), the quasi-three-dimensional data still contains enough information to utilize the drift-volume technique. Each two-dimensional plane contains tracked particles with an associated thickness, allowing them to occupy a three-dimensional space. Because the drift volume calculation only requires knowledge of particle displacements in a single dimension, removing the out-of-plane velocity component still allows for the one-dimensional drag force on an accelerating body to be measured. 


\subsection{Future Work}

While this thesis demonstrated the feasibility of using a new technique to extract instantaneous forces on submersed bodies, there is still much work yet to be done in this area, such as the sensitivity to measurement uncertainty and quantitative comparisons to existing techniques.

A useful development for the proposed force estimation method is the quantification of experimental parameters and their effects on the final force output. Error evaluation associated with a large FoV versus the trade-off of high resolution is the most prominent experimental parameter to control. Even though the requirement for high spatial resolution has been reduced, it has not been made non-existent. This control may also determine the range of $N$ reference planes that succeeds in estimating accurate forces. Determining the limiting cases for functionality of the described technique is a useful endeavour.

The Lagrangian aspects of the drift-volume force estimation technique are of unique interest when compared to Eulerian techniques. It may be possible to use the Lagrangian data to introduce the origin of force and spatial loading. The current state of the method is one that only measures the total force at each time step from a single integration over all space. It may also be possible to disassemble the total force into spatial loading elements through the use of other Lagrangian analysis. For example, the state of the wake, the force history and the drift volume could be combined using particle path information to draw conclusions about the dynamics of vortex interaction.

The added-mass force is not limited to one-dimensional translation, as used in the current study, but can also be applied to objects undergoing a combination of linear 
and angular accelerations. The added-mass tensor has six components, only three of which are linear, hence there also exist unsteady forces due to angular accelerations that can be measured using a variation of the drift-volume approach presented. No work has yet been done to investigate the relationship between Lagrangian particle displacement and the angular analogue to added mass. Studies in potential flow simulations and in experiments could prove useful to understanding and measuring the forces on arbitrary motions that utilize multiple degrees of freedom.

One last relevant future development of this force estimation algorithm is application to non-zero initial conditions. In the current test case, a body was accelerated from rest, meaning that all surrounding fluid was initially at rest. This test case represents the best case scenario for measuring the unsteady effects of acceleration through a fluid as all fluid motion can be attributed to the unsteady reaction force. A more complex example of added-mass force calculation comes from non-zero initial conditions, where a fully-developed wake already exists at the beginning of an acceleratory motion. The drift-volume method would likely need to be modified to account for the non-zero initial velocity of the surrounding fluid. The method should include an offset corresponding the initial velocity of the body so that only the unsteadiness of the motion is captured by the multi-plane drift volume. The added-mass force contribution can then be added onto the force recorded prior to the unsteady motion in order to estimate the entire force felt by the submersed object. 


\section{Bibliography}

Adrian, R. J. (1997). "Dynamic ranges of velocity and spatial resolution of particle image velocimetry". In: Measurement Science and Technology 8.12, p. 1393. URL: http://stacks.iop.org/0957-0233/8/i=12/a=003.

Ahsan, S. N. and M. Aureli (2017). "Nonlinear oscillations of shape-morphing submerged structures: Control of hydrodynamic forces and power dissipation via active flexibility". In: Journal of Fluids and Structures 74, pp. 35 -52. ISSN: 08899746. DOI: https://doi.org/10.1016/j.jfluidstructs . 2017.06.010. URL: http://www.sciencedirect.com/science/article/pii/S0889974616303693.

Ansari, S. A., R. Żbikowski, and K. Knowles (2006). "Aerodynamic modelling of insect-like flapping flight for micro air vehicles". In: Progress in Aerospace Sciences 42.2, pp. 129-172. ISSN: 0376-0421. URL: http : //www . sciencedirect. com / science/article/pii/S0376042106000467.

Babinsky, H., R. J. Stevens, A. R. Jones, L. P. Bernal, and M. V. Ol (2016). "Low Order Modelling of Lift Forces for Unsteady Pitching and Surging Wings". In: AIAA SciTech Forum. American Institute of Aeronautics and Astronautics. DOI: 10.2514/6.2016-0290. URL: https://doi.org/10.2514/6.2016-0290. 
Baik, Y., L. Bernal, K. Granlund, and M. Ol (2012). "Unsteady force generation and vortex dynamics of pitching and plunging aerofoils". In: Journal of Fluid Mechanics 709, pp. 37-68.

Batchelor, G. (1967). An Introduction to Fluid Dynamics. Cambridge Mathematical Library. Cambridge University Press. ISBN: 9780521663960. URL: https ://books . google.ca/books?id=Rla70ihRvUgC.

Benedict, M., D. Coleman, D. B. Mayo, and I. Chopra (2016). "Experiments on Rigid Wing Undergoing Hover-Capable Flapping Kinematics at Micro-Air-Vehicle-Scale Reynolds Numbers". In: AIAA Journal 54.4, pp. 1145-1157. ISSN: 0001-1452. DOI: 10.2514/1.j052947. URL: https://doi.org/10.2514/1. J052947.

Benjamin, T. B. (1986). "Note on added mass and drift". In: Journal of Fluid Mechanics 169, 251256. DOI: 10.1017/S0022112086000617.

Betz, A. (1925). "A method for the direct determination of profile drag (in German)". In: Zeitschrift für Flugtechnik und Motorluftschifffahrt 16, pp. 42-44. DOI: 10. 1007/s00348-011-1241-9.

Bos, F. M., D. Lentink, B. W. van Oudheusden, and H. Bijl (2008). "Influence of wing kinematics on aerodynamic performance in hovering insect flight". In: Journal of Fluid Mechanics 594, 341368. DOI: 10.1017/S0022112007009172.

Brennan, C. E. (1982). A Review of Added Mass and Fluid Inertial Forces. Tech. rep. CR 82.010. Department of the Navy.

Burden, R., J. Faires, and A. Burden (2015). Numerical Analysis. Cengage Learning. ISBN: 9781305465350 . URL: https : / / books . google . ca/books?id=2nZvCgAAQBAJ. 
Clift, R., J. Grace, and M. Weber (1978). Bubbles, Drops, and Particles. Academic Press. ISBN: 9780121769505. URL: https : / / books . google. ca / books ? id= n8gRAQAAIAAJ.

Dabiri, J. O. (2005). "On the estimation of swimming and flying forces from wake measurements". In: Journal of Experimental Biology 208, pp. 3519-3532. DOI: $10.1242 / \mathrm{jeb} .01813$.

d'Alembert, J. (1752). Essai d'une nouvelle thorie de la rsistance des fluides. Ed. by D. L'aine. URL: http://catalogue.bnf .fr/ark:/12148/cb30009144f.

Daniel, T. L. (1984). "Unsteady Aspects of Aquatic Locomotion". In: American Zoologist 24.1, pp. 121-134. ISSN: 00031569. URL: http://www.jstor.org/stable/ 3882757.

Darwin, C. (1953). "Note on hydrodynamics". In: Mathematical Proceedings of the Cambridge Philosophical Society 49.2, 342354. DOI: 10.1017/S0305004100028449.

Eames, I., S. E. Belcher, and J. C. R. Hunt (1994). "Drift, partial drift and Darwin's proposition". In: Journal of Fluid Mechanics 275, 201223. DOI: 10.1017/ S0022112094002338.

Eloy, C. (2013). "On the best design for undulatory swimming". In: Journal of Fluid Mechanics 717, pp. 48-89. DOI: 10.1017/jfm.2012.561.

Elsinga, G. E., F. Scarano, B. Wieneke, and B. W. van Oudheusden (2006). "Tomographic Particle Image Velocimetry". In: Exp Fluids 41, pp. 933-947. DOI: $10.1007 / \mathrm{s} 00348-006-0212-\mathrm{z}$.

Fernando, J. N. and D. E. Rival (2016). "Reynolds-number scaling of vortex pinch-off on low-aspect-ratio propulsors". In: Journal of Fluid Mechanics 799. 
Fernando, J. N. and D. E. Rival (2017). "On the dynamics of perching manoeuvres with low-aspect-ratio planforms". In: Bioinspiration \& Biomimetics 12.4, p. 046007. URL: http://stacks.iop.org/1748-3190/12/i=4/a=046007.

Fernando, J. N. (2017). "Vortex evolution in the wake of accelerating low-aspect-ratio plates and three-dimensional bodies of revolution". PhD thesis. Queen's University.

Garca, J., J. Muoz-Paniagua, and A. Crespo (2017). "Numerical study of the aerodynamics of a full scale train under turbulent wind conditions, including surface roughness effects". In: Journal of Fluids and Structures 74, pp. 1 -18. ISSN: 08899746. DOI: https://doi.org/10.1016/j.jfluidstructs . 2017.07.007. URL: http://www.sciencedirect.com/science/article/pii/S0889974617301007.

Gent, P. L. van, D. Michaelis, B. W. van Oudheusden, P. É. Weiss, R. de Kat, A. Laskari, Y. J. Jeon, L. David, D. Schanz, F. Huhn, S. Gesemann, M. Novara, C. McPhaden, N. J. Neeteson, D. E. Rival, J. F. G. Schneiders, and F. F. J. Schrijer (2017). "Comparative assessment of pressure field reconstructions from particle image velocimetry measurements and Lagrangian particle tracking". In: Experiments in Fluids 58.4, p. 33. ISSN: 1432-1114. DOI: 10.1007/s00348-0172324-z. URL: https://doi.org/10.1007/s00348-017-2324-z.

Green, M. A., C. W. Rowley, and A. J. Smits (2011). "The unsteady three-dimensional wake produced by a trapezoidal pitching panel". In: Journal of Fluid Mechanics 685, 117145. DOI: $10.1017 / \mathrm{jfm} .2011 .286$.

Ikeda, S. and Y. Yamamoto (1981). "Lift forces on cylinders in oscillatory flows". In: Report of Department Foundation of Engineering and Coastal Engineering 10, pp. $1-16$. 
Jones, B. M. (1936). "Measurement of Profile Drag by the Pitot-Traverse Method". In: $A R C R E M$ No. 1688.

Kähler, C. J., S. Scharnowski, and C. Cierpka (2012). "On the Uncertainty of Digital PIV and PTV Near Walls". In: Experiments in Fluids 52.6, pp. 1641-1656. DOI: $10.1007 / \mathrm{s} 00348-012-1307-3$.

Karanfilian, S. K. and T. J. Kotas (1978). "Drag on a sphere in unsteady motion in a liquid at rest". In: Journal of Fluid Mechanics 87.1, 8596. DoI: 10.1017/ S0022112078002943.

Küssner, H. G. (1936). "Zusammenfassender Bericht ber den instationren Auftrieb von Flgeln (Summary report on the instationary lift of wings)". In: Luftfahrtforschung 13.12, pp. 410-424.

Lighthill, M. J. (1956). "Drift". In: Journal of Fluid Mechanics 1.1, 3153. DOI: 10. 1017/S0022112056000032.

Manar, F., P. Mancini, D. Mayo, and A. R. Jones (2016). "Comparison of Rotating and Translating Wings: Force Production and Vortex Characteristics". In: AIAA Journal 54.2, pp. 519-530. ISSN: 0001-1452. DOI: 10 . 2514/1 . j054422. URL: https://doi.org/10.2514/1. J054422.

Mancini, P., F. Manar, K. Granlund, M. V. Ol, and A. R. Jones (2015). "Unsteady aerodynamic characteristics of a translating rigid wing at low Reynolds number". In: Physics of Fluids 27.12, p. 123102. DOI: 10.1063/1.4936396. eprint: http: //dx.doi.org/10.1063/1.4936396. URL: http://dx.doi.org/10.1063/1. 4936396.

Maull, D. and M. Milliner (1978). "Sinusoidal flow past a circular cylinder". In: Coastal Engineering 2, pp. 149 -168. ISSN: 0378-3839. DOI: http://dx.doi. 
org/10.1016/0378-3839(78) 90013-3. URL: http://www. sciencedirect. com/ science/article/pii/0378383978900133.

Mohebbian, A. and D. Rival (2012). "Assessment of the derivative-moment transformation method for unsteady-load estimation". In: Experiments in Fluids 53, pp. 319-330.

Motta, V., A. Guardone, and G. Quaranta (2015). "Influence of airfoil thickness on unsteady aerodynamic loads on pitching airfoils". In: Journal of Fluid Mechanics 774, pp. 460-487. ISSN: 0022-1120. DOI: 10.1017/jfm.2015.280. URL: https : / / www . cambridge . org / core / article/influence - of - airfoilthickness - on - unsteady - aerodynamic - loads - on - pitching - airfoils / FC5F8C16F88FD26EDFCC7A43342D12FD.

Noca, F., D. Shiels, and D. Jeon (1997). "Measuring Instantaneous Fluid Dynamic Forces on Bodies, Using Only Velocity Fields and Their Derivatives". In: Journal of Fluids and Structures 11, pp. 345-350.

- (1999). "A Comparison of Methods for Evaluating Time-Dependent Fluid Dynamic Forces on Bodies, Using only Velocity Fields and Their Derivatives". In: Journal of Fluids and Structures 13, pp. 551-578.

Odar, F. and W. S. Hamilton (1964). "Forces on a sphere accelerating in a viscous fluid". In: Journal of Fluid Mechanics 18.2, 302314. DOI: 10.1017/S0022112064000210.

Pitt Ford, C. W. and H. Babinsky (2013). "Lift and the Leading Edge Vortex". In: Journal of Fluid Mechanics 720, pp. 280-313.

Polet, D. T. and D. E. Rival (2015). "Rapid area change in pitch-up manoeuvres of small perching birds". In: Bioinspiration $\mathscr{E}$ Biomimetics 10.6, p. 066004. URL: http://stacks. iop.org/1748-3190/10/i=6/a=066004. 
Raffel, M., C. Willert, S. Wereley, and J. Kompenhans (2007). Particle image velocimetry: a practical guide. 2nd ed. Springer, pp. 164-176.

Rival, D. E. and B. v. Oudheusden (2017). "Load-estimation techniques for unsteady incompressible flows". In: Experiments in Fluids 58.3, p. 20. ISSN: 1432-1114. DOI: 10 . 1007/s00348-017-2304-3. URL: http://dx . doi .org/10 . 1007/s00348017-2304-3.

Saffman, P. (1992). Vortex Dynamics. Cambridge Monographs on Mechanics and Applied Mathematics. Cambridge University Press. ISBN: 9780521477390.

Sarpkaya, T. (1963). "Lift, Drag, and Added-Mass Coefficients for a Circular Cylinder Immersed in a Time-Dependent Flow". In: Journal of Applied Mechanics 30.1, pp. 13-15. ISSN: 0021-8936. DOI: 10.1115/1 .3630062. URL: http://dx . doi . org/10.1115/1.3630062.

Schanz, D., S. Gesemann, and A. Schröder (2016). "Shake-The-Box: Lagrangian particle tracking at high particle image densities". In: Experiments in Fluids 57:70.

Sciacchitano, A. and B. Wieneke (2016). "PIV uncertainty propagation". In: Measurement Science and Technology 27.8, p. 084006. URL: http://stacks .iop. org $/ 0957-0233 / 27 / i=8 / a=084006$.

Sciacchitano, A., B. Wieneke, and F. Scarano (2013). "PIV uncertainty quantification by image matching". In: Measurement Science and Technology 24.4, p. 045302. URL: http://stacks . iop.org/0957-0233/24/i=4/a=045302.

Theodorsen, T (1935). "General theory of aerodynamic instability and the mechanism of flutter". In: NACA Report 496. 
Tropea, C., A. Yarin, and J. F. Foss (2007). Springer Handbook of Experimental Fluid Mechanics. Ed. by C. Tropea, A. Yarin, and J. F. Foss. 1st ed. Springer-Verlag Berlin Heidelberg. ISBN: 978-3-662-49162-1.

Wang, Q., J. F. L. Goosen, and F. van Keulen (2016). "A predictive quasi-steady model of aerodynamic loads on flapping wings". In: Journal of Fluid Mechanics 800, 688719. DOI: $10.1017 / \mathrm{jfm} .2016 .413$.

Westerweel, J. (2008). "On velocity gradients in PIV interrogation". In: Experiments in Fluids 44.5, pp. 831-842. ISSN: 1432-1114. DOI: 10.1007/s00348-007-0439-3. URL: http://dx.doi .org/10.1007/s00348-007-0439-3.

Weymouth, G. and M. S. Triantafyllou (2012). "Global vorticity shedding for a shrinking cylinder". In: Journal of Fluid Mechanics 702, pp. 470-487. DoI: http://dx. doi .org/10.1017/jfm. 2012. 200. eprint: http://journals . cambridge .org/ abstract_S0022112012002005.

- (2013). "Ultra-fast escape of a deformable jet-propelled body". In: Journal of Fluid Mechanics 721, pp. 367-385. DoI: http://dx.doi .org/10.1017/jfm. 2013.65. eprint: http://journals.cambridge.org/abstract_S0022112013000657.

Wieneke, B. (2015). "PIV uncertainty quantification from correlation statistics". In: Measurement Science and Technology 26.7, p. 074002. URL: http://stacks.iop. org $/ 0957-0233 / 26 / i=7 / a=074002$.

Willert, C. E. and M. Gharib (1991). "Digital particle image velocimetry". In: Experiments in Fluids 10.4, pp. 181-193. ISSN: 1432-1114. DOI: 10.1007/BF00190388. URL: http://dx.doi.org/10.1007/BF00190388.

Wu, J.-Z., Z.-L. Pan, and X.-Y. Lu (2005). "Unsteady fluid-dynamic force solely in terms of control-surface integral". In: Physics of Fluids 17, 098102-1. 
Wu, J., H. Ma, and J. Zhou (2006). Vorticity and Vortex Dynamics. Lecture notes in mathematics. Springer Berlin Heidelberg. ISBN: 9783540290285.

Yih, C.-S. (1985). "New derivations of Darwin's theorem". In: Journal of Fluid Mechanics 152, 163172. DOI: 10.1017/S0022112085000623. 


\section{Appendix A}

\section{Uncertainty Analysis}

In this appendix, the uncertainty in the PIV measurement analysis and the measured force are quantified.

\section{A.1 PIV Uncertainty}

In this section, a brief description of the PIV uncertainty presented in Section 4.2 is detailed.

The PIV software used can calculate particle positions to within sub-pixel accuracy, at approximately $\pm 0.1 \mathrm{px}$. With an average pixel displacement between frames of $5 \mathrm{px}$, the resulting error in particle displacement $\sigma_{d}$ is roughly $2 \%$. The corresponding uncertainty in the velocity field $\sigma_{v}$ is calculated by:

$$
\sigma_{v}=|v| \sqrt{\left|\frac{\sigma_{d}}{\Delta d}\right|^{2}+\left|\frac{\sigma_{t}}{\Delta t}\right|^{2}}
$$

where $v$ is the velocity, $\frac{\sigma_{d}}{\Delta d}$ is the $2 \%$ uncertainty in particle positions, and $\frac{\sigma_{t}}{\Delta t}$ is the uncertainty in the time delay between sequential images, which is assumed to be negligible. A safe assumption, after taking into consideration the phase-averaged results 
from 10 identical runs and bias errors in regions of high shear, is that the maximum uncertainty in the velocity field is double the uncertainty in particle positions, at $4 \%$.

Using the assumptions presented by Sciacchitano and Wieneke (2016), the uncertainty in the vorticity field $U_{\omega}$ takes on the form:

$$
U_{\omega}=\frac{U}{d} \sqrt{1-\rho(2 d)}
$$

where $U$ is the uncertainty of the velocity field, $d$ is the grid spacing and $\rho(2 d)$ is the cross-correlation coefficient. When this equation is applied to the data presented by Sciacchitano and Wieneke (2016) for a correlation window size of $32 \times 32 \mathrm{px}^{2}$ at $75 \%$ overlap, the corresponding average error in vorticity is approximately $8 \%$. In areas of high shear and near walls, the uncertainty in vorticity is considerably higher, as explained by Kähler et al. (2012).

\section{A.2 Measured force uncertainty}

The force transducer used to measure the drag force on the acceleration plate had a resolution of $0.125 \mathrm{~N}$. The force data was averaged over 20 identical runs yielding a total uncertainty of:

$$
\sigma_{F}=\frac{0.125 \mathrm{~N}}{\sqrt{20}}=0.028 \mathrm{~N},
$$

which, when compared to the peak force of $39 \mathrm{~N}$, equates to a $0.07 \%$ uncertainty. 


\section{Appendix B}

\section{MATLAB Code}

\section{B.1 Algorithm Description}

In this appendix, the MATLAB scripts used in the multi-plane drift-volume force estimation technique are given. A full description of the inner workings of the algorithm is provided within the comments of each script.

In the following sections, the overall algorithm is explained step-by-step, delineating the exact required format of input data, the order of execution of MATLAB functions, and the format of the output data. The scripts generate folders within the root directory for efficient data organization. The output data is saved at each time step into folders corresponding to the number of reference planes $N$ selected and can be later used to visualize the drift-volume development over time. Each '.mat' data file saved by the MATLAB scripts contains spatial coordinates for all particles corresponding to positive, negative and net drift volumes. Two different types of MATLAB '.mat' data files are saved for a single run of the algorithm, in which a single $N$ value has been chosen. 
The first type of save file is labeled as "RG_plane\#_t\#.mat" where the first number sign is the plane index, between 1 and $N$ and the second number sign is the timestep number. This data file contains the Cartesian coordinates for all positive and negative drift volume particles. The variables Xgreen and Ygreen represent the $x$ and $y$ coordinates for the positive drift contributing particles. Similarly, the variables Xred and Yred represent the $x$ and $y$ coordinates for the negative drift contributing particles.

The second type of save file is labeled as "DV_plane\#.mat" where the number sign is the plane index. These data files contains variables for the positive $\mathrm{Vg}$, negative $\mathrm{Vr}$, and net $\mathrm{V}$ drift volume contributions $\Omega_{d_{n}}$ from a single reference plane.

\section{B.2 Calculation of Drift Volume}

This section describes the algorithm used to calculate the multi-plane drift volume from a dataset of particle tracks.

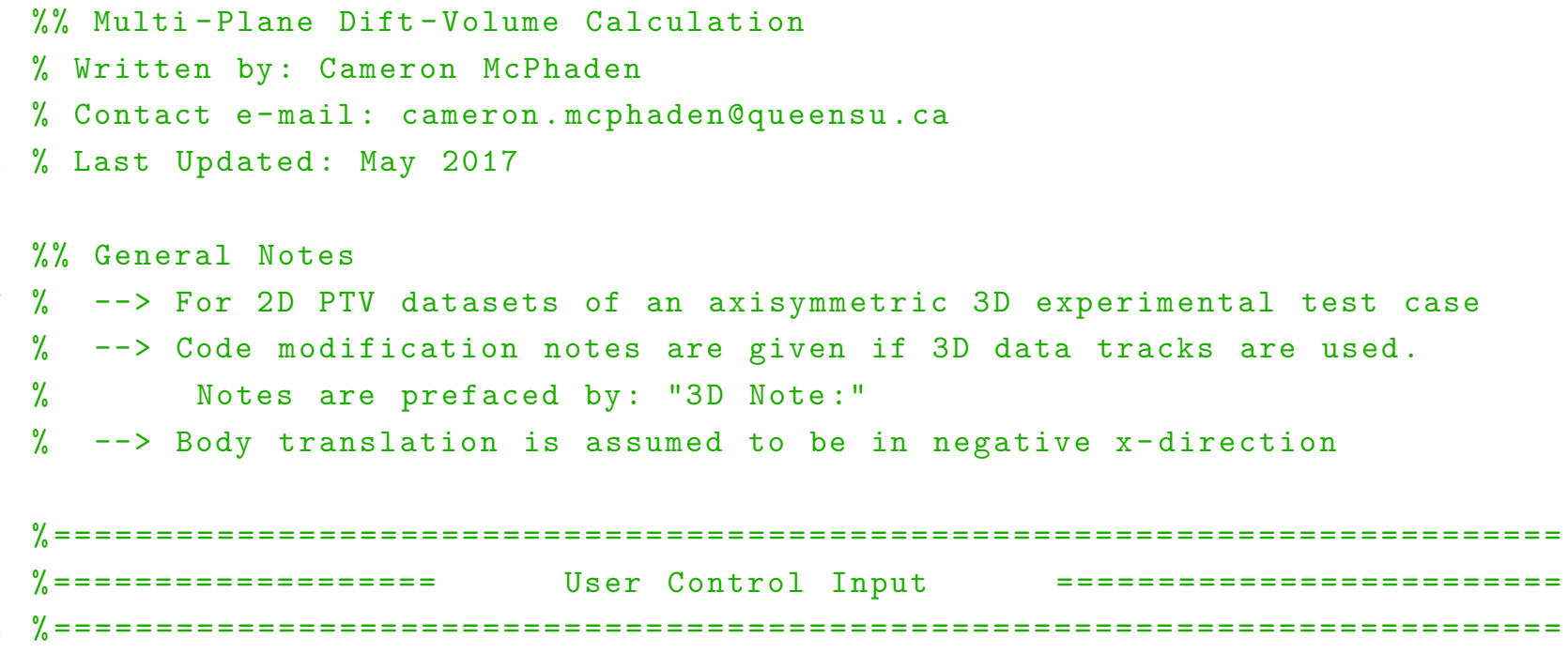




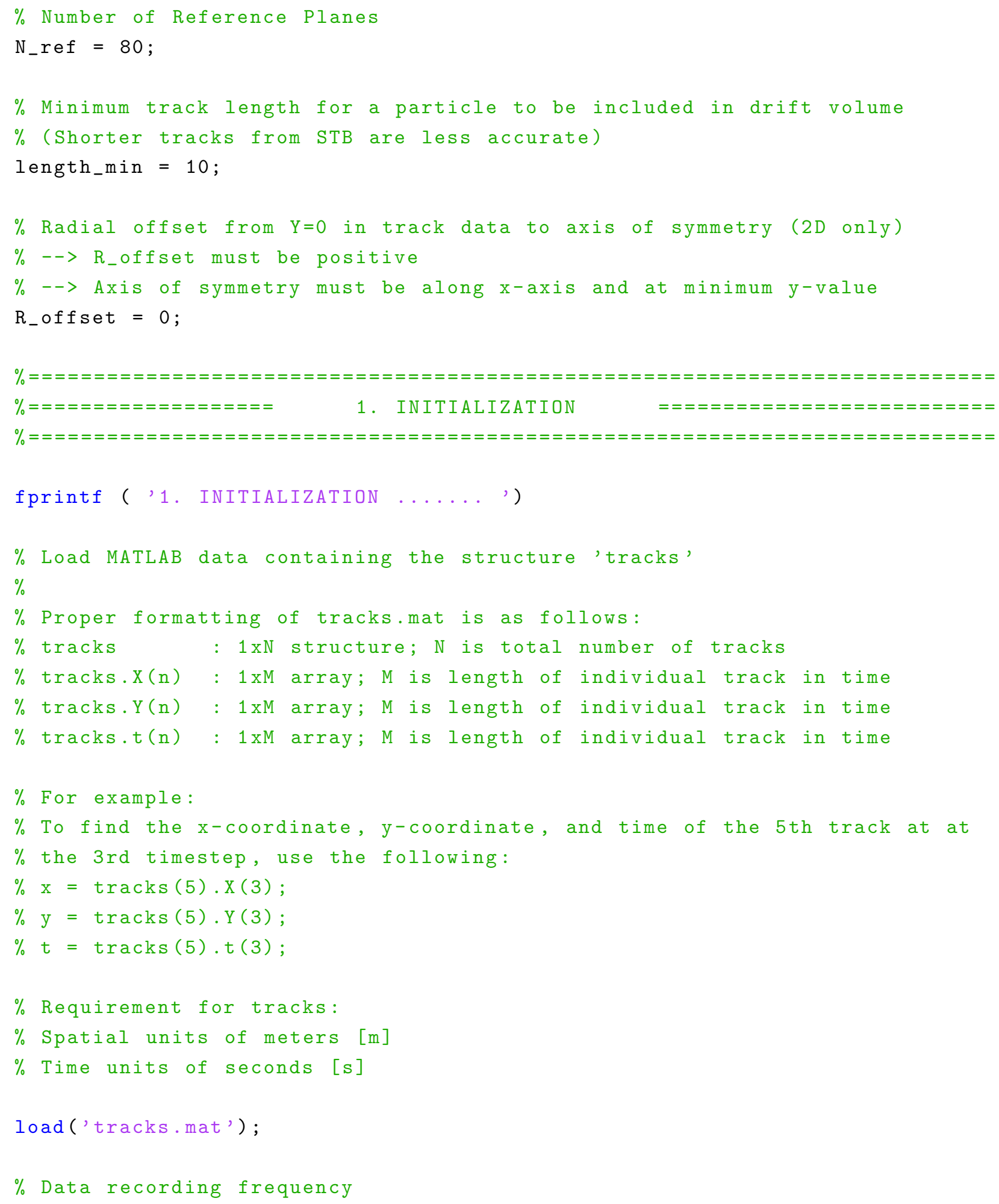




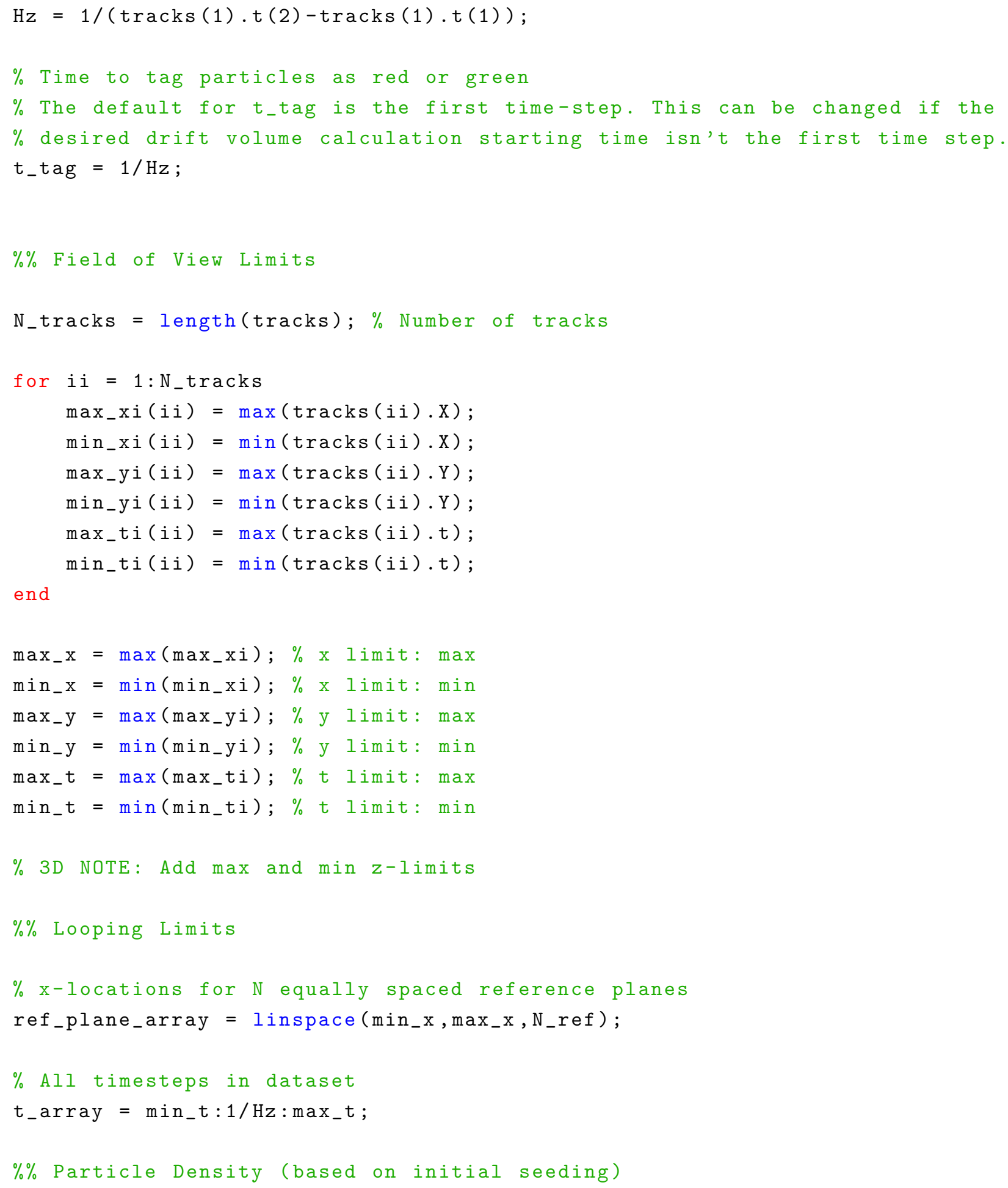




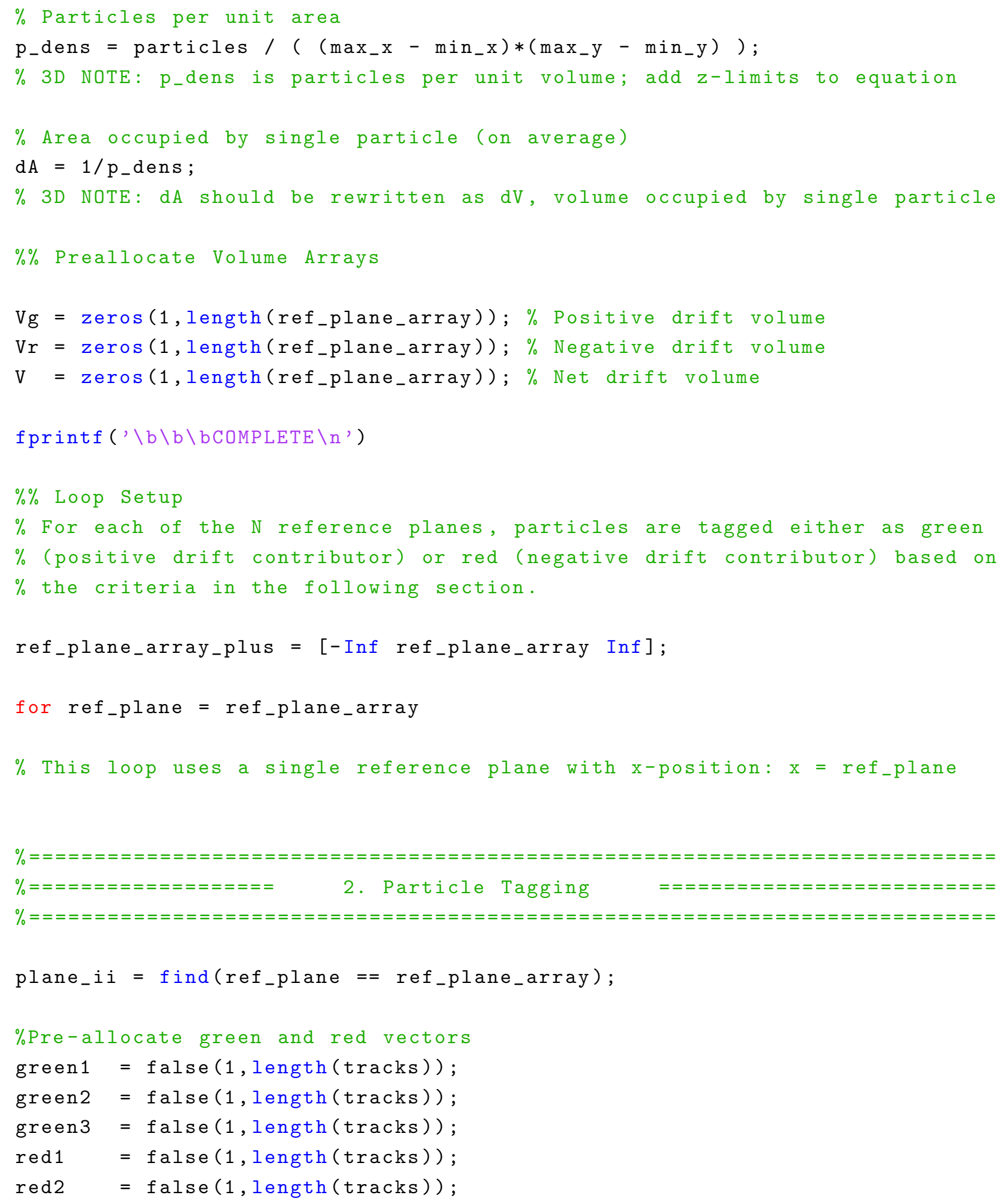




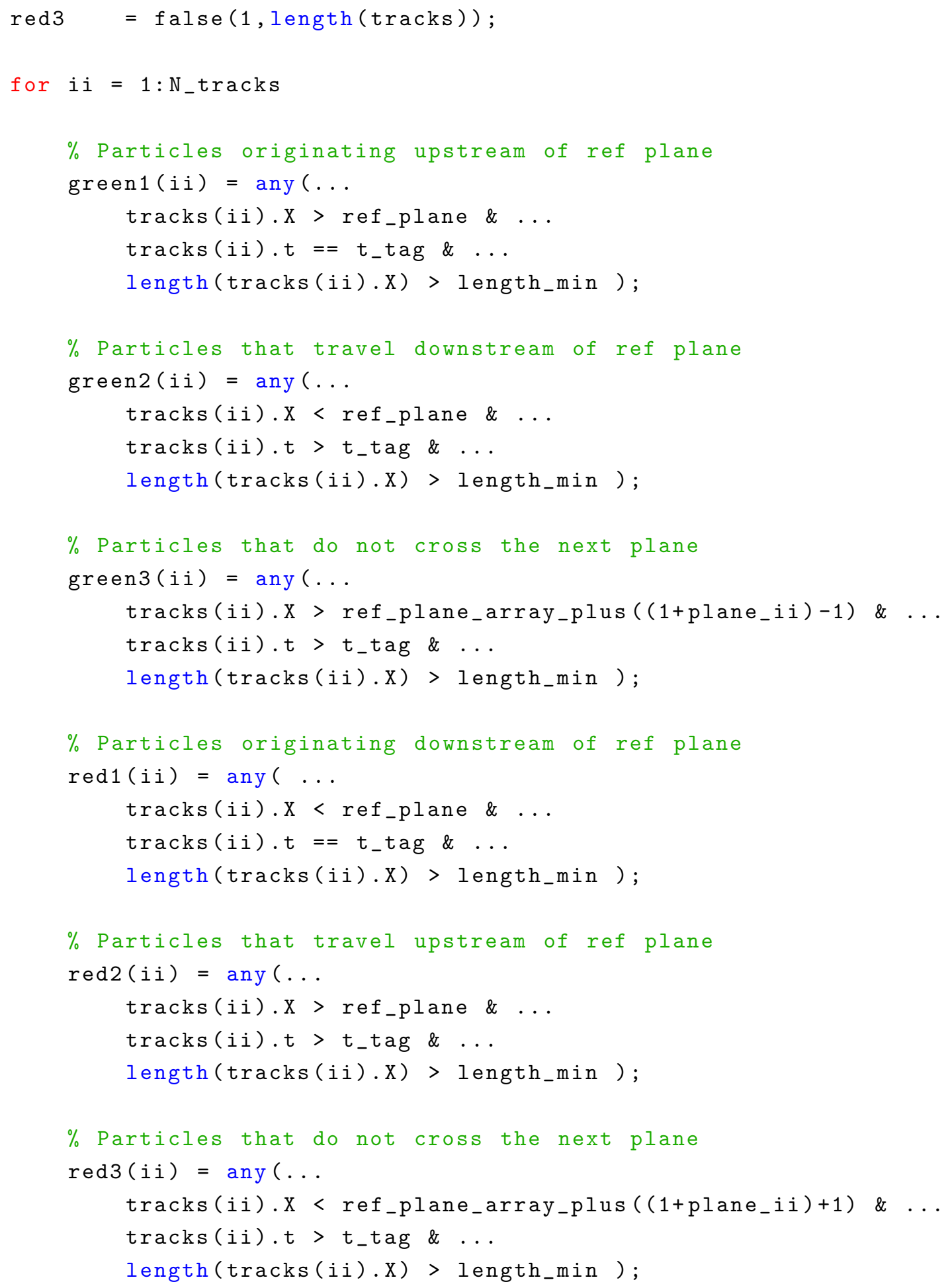

174
175 


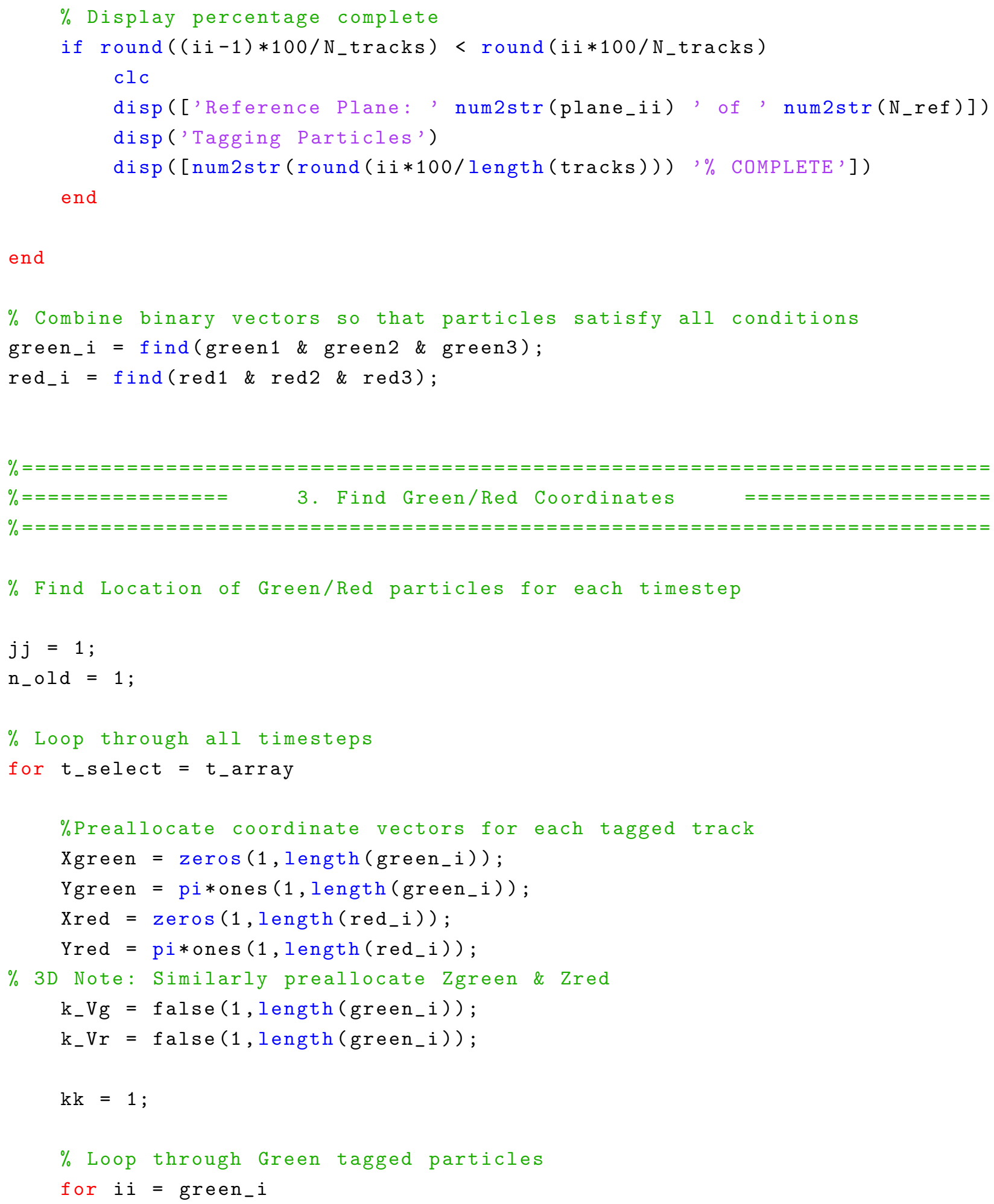




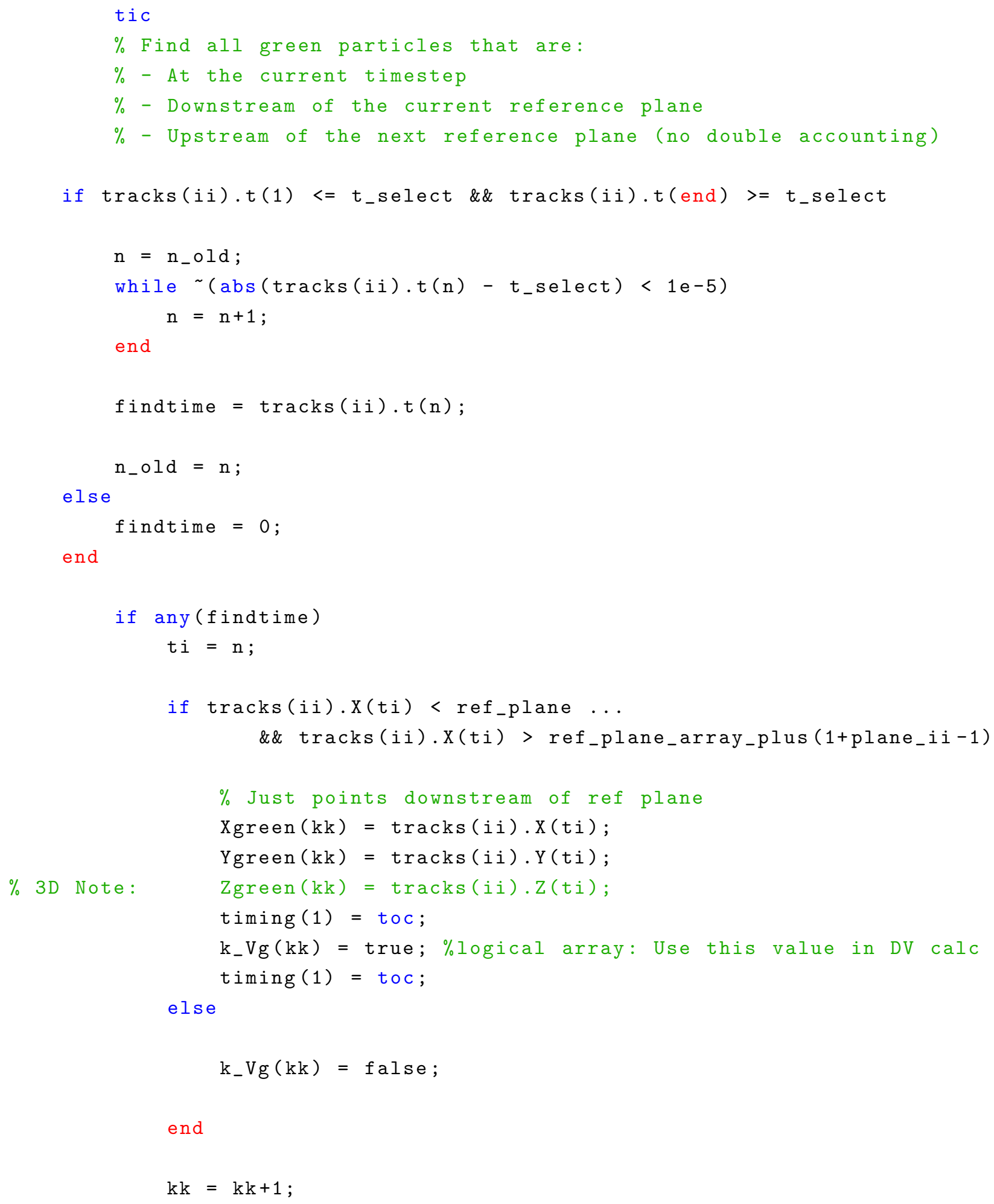


256

257

258

259

260

261

262

263

264

265

266

267

268

269

270

271

272

273

274

275

276

277

278

279

280

281

282

283

284

285

286

287

288

289

290

291

292

293

294

295

end

end

$\mathrm{kk}=1$;

for $i i=$ red $_{-} i$

$\%$ Find all Red particles that are:

$\%$ - At the current timestep

$\%$ - Upstream of the current reference plane

$\%$ - Downstream of the next reference plane (no double accounting)

if $\operatorname{any}(f i n d t i m e)$

$t i=n ;$

if length (tracks $(i i) . X)>t i \ldots$

$\& \& \operatorname{tracks}(i i) . X(t i)>$ ref_plane...

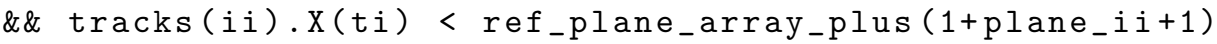

\% Just points upstream of ref plane

$\operatorname{Xred}(\mathrm{kk})=\operatorname{tracks}(\mathrm{i} i) \cdot \mathrm{X}(\mathrm{ti})$;

$\operatorname{Yred}(\mathrm{kk})=\operatorname{tracks}(\mathrm{i} i) \cdot \mathrm{Y}(\mathrm{t} i)$;

$\%$ 3D Note: $\quad$ Zred $(\mathrm{kk})=\operatorname{tracks}(\mathrm{i} i) \cdot \mathrm{Z}(\mathrm{ti})$;

$\mathrm{k}_{-} \operatorname{Vr}(\mathrm{kk})=$ true; \%logical array: Use this value in DV calc

else

$\mathrm{k}_{-} \operatorname{Vr}(\mathrm{kk})=\mathrm{false}$

end

$\mathrm{kk}=\mathrm{kk}+1$

end

end

if $\left.\operatorname{sum}_{(\text {green_i }}\right)==0$ 


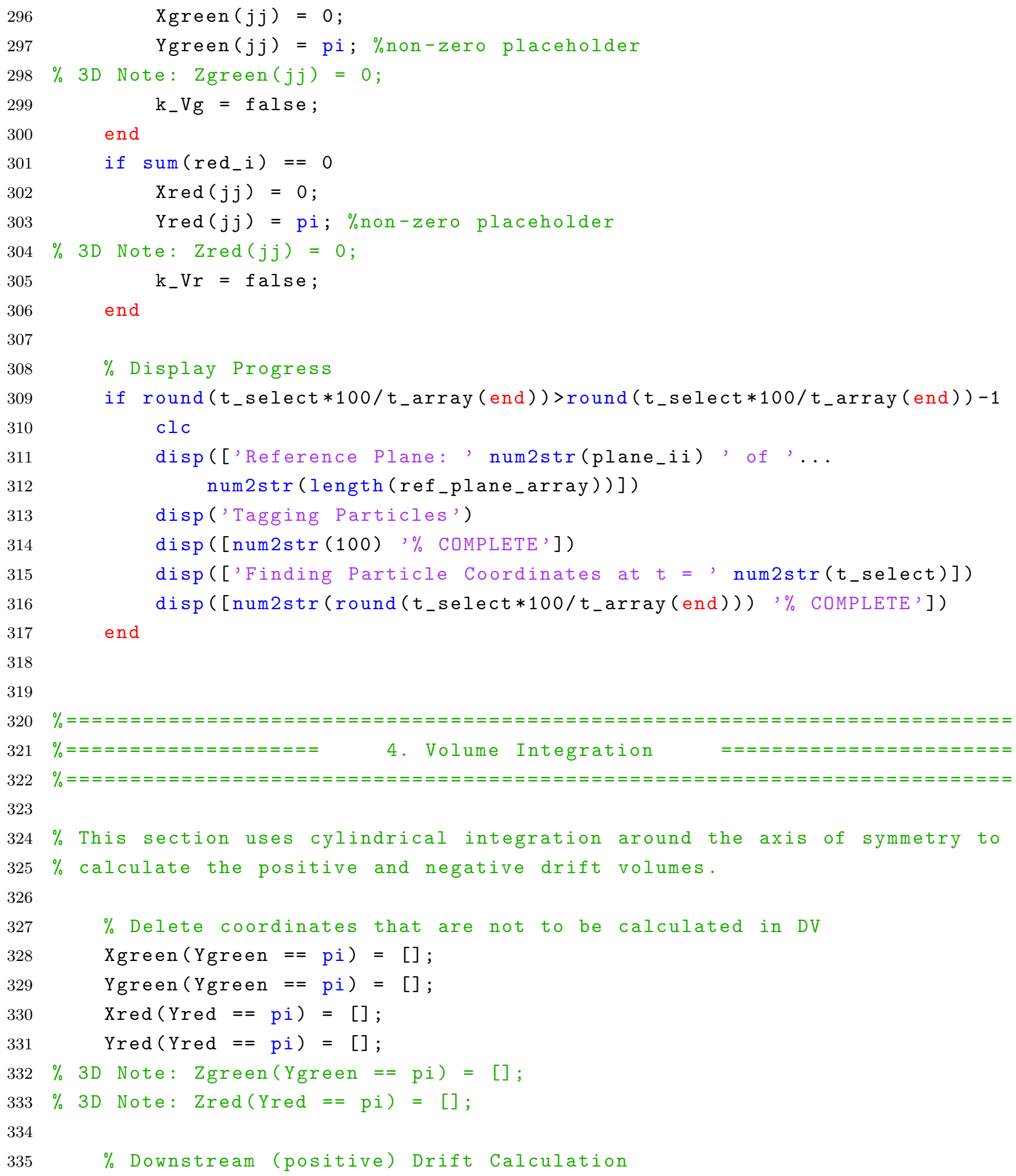




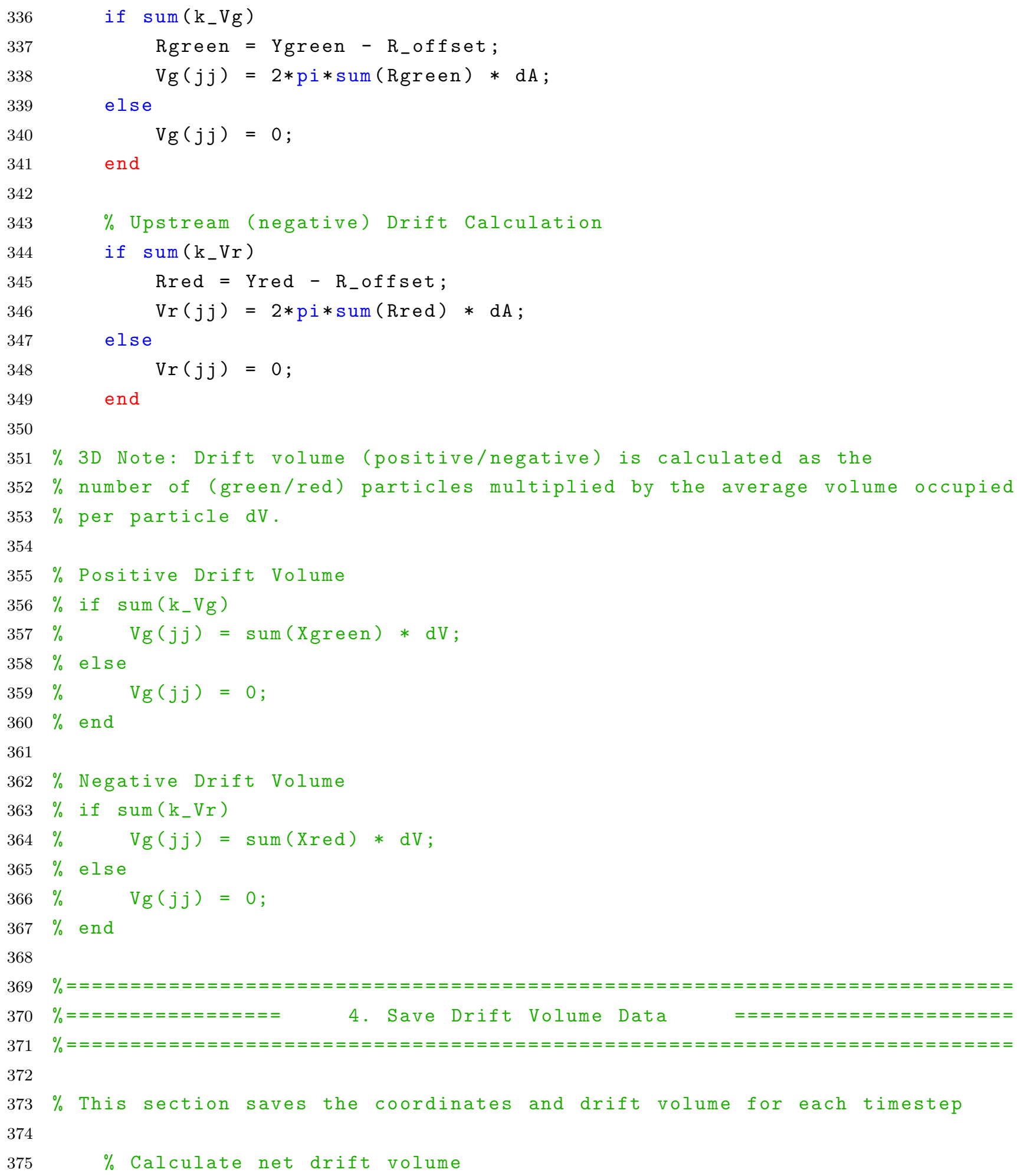




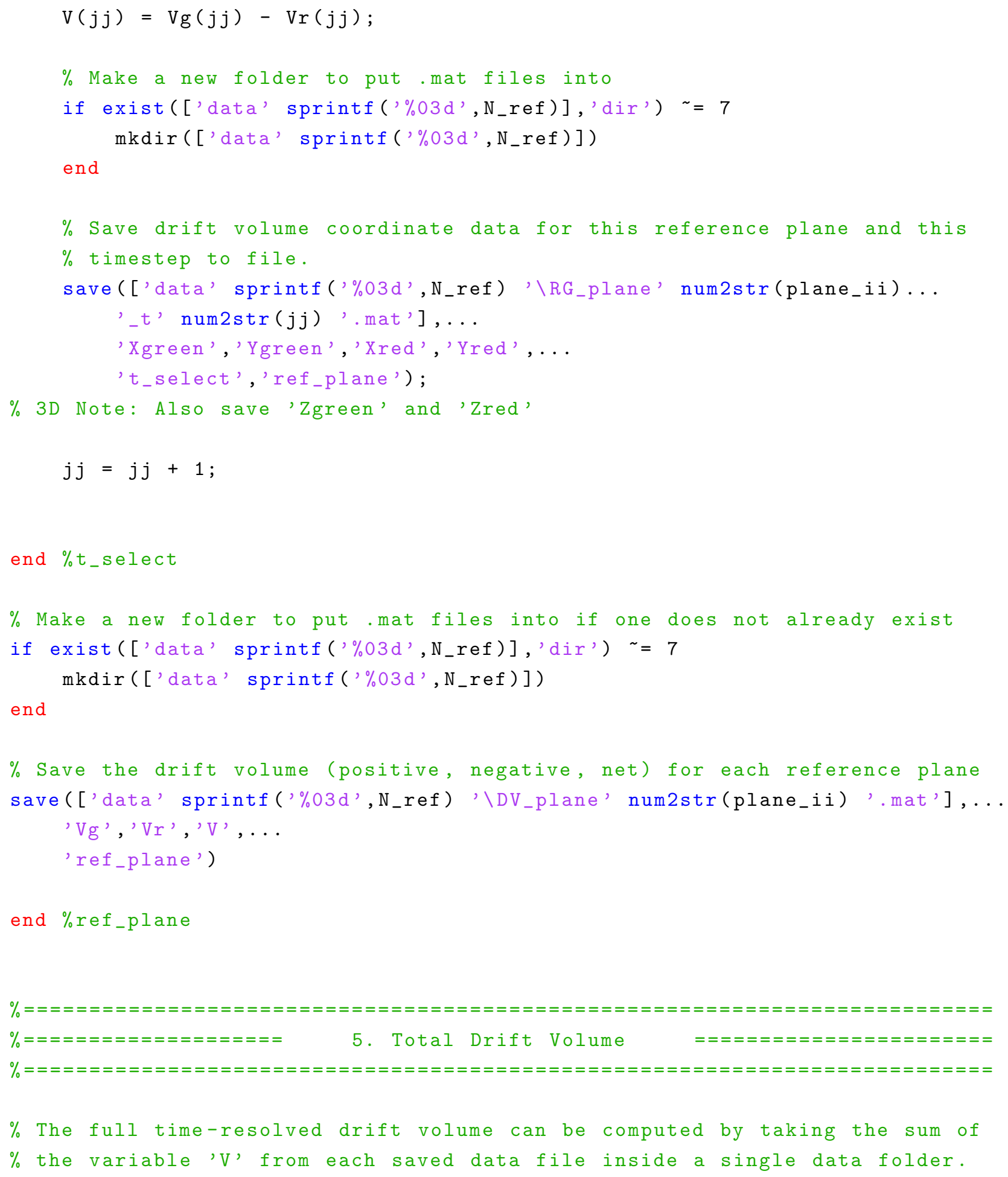




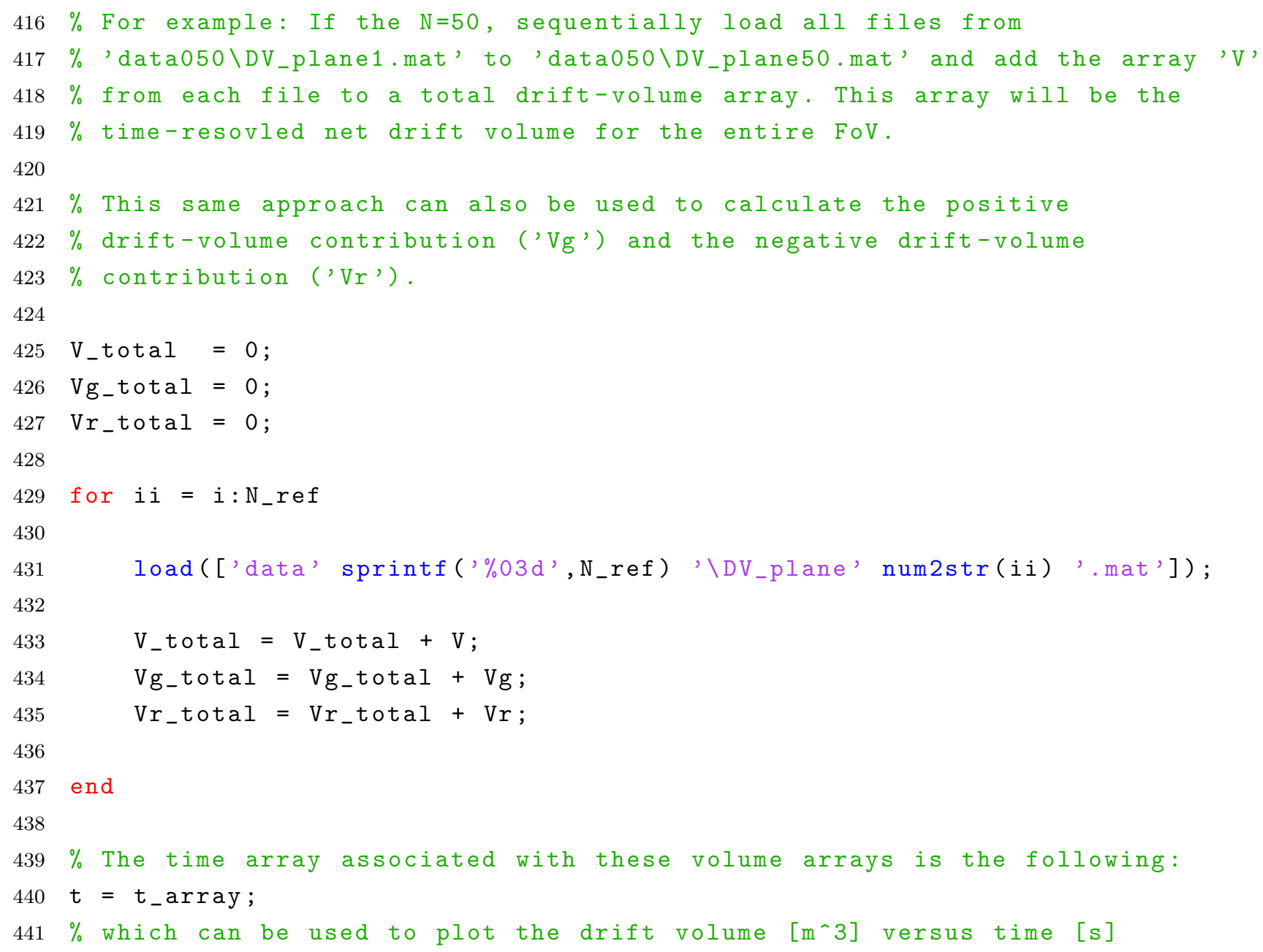




\section{B.3 Force Calculation}

This section describes the algorithm used to calculate the force $\mathbf{F}$ acting on a translating body using the drift volume previously calculated.

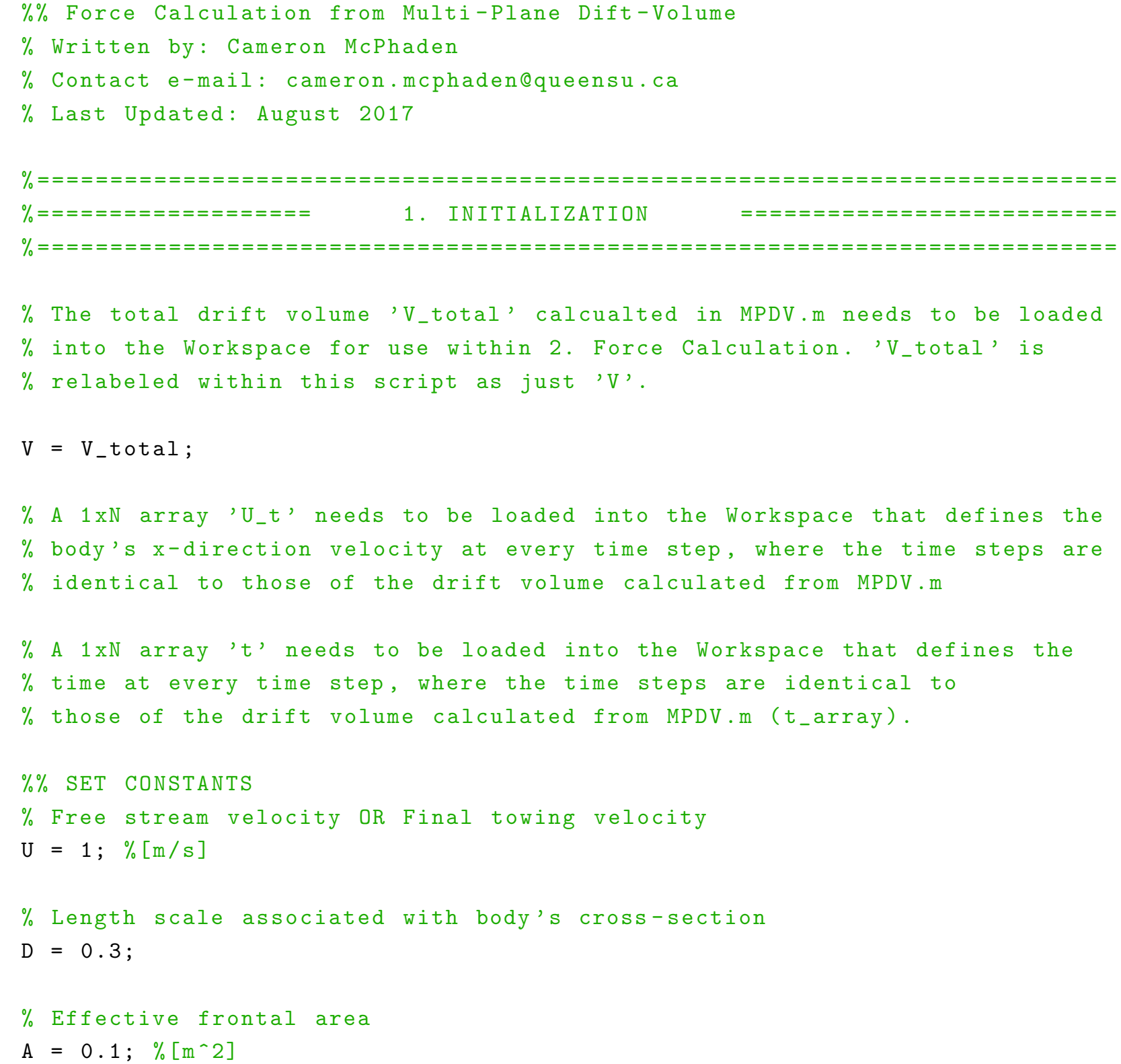




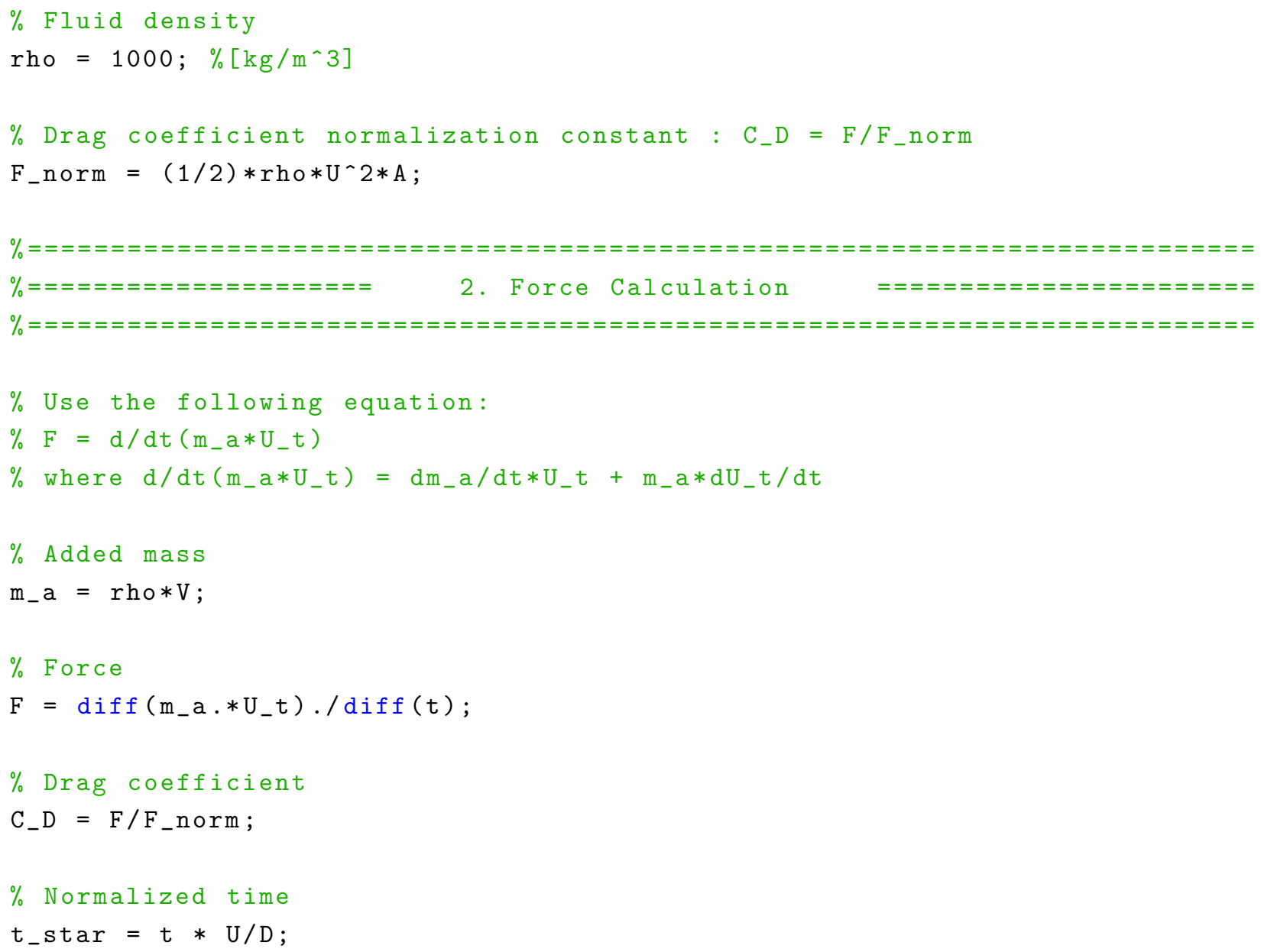

